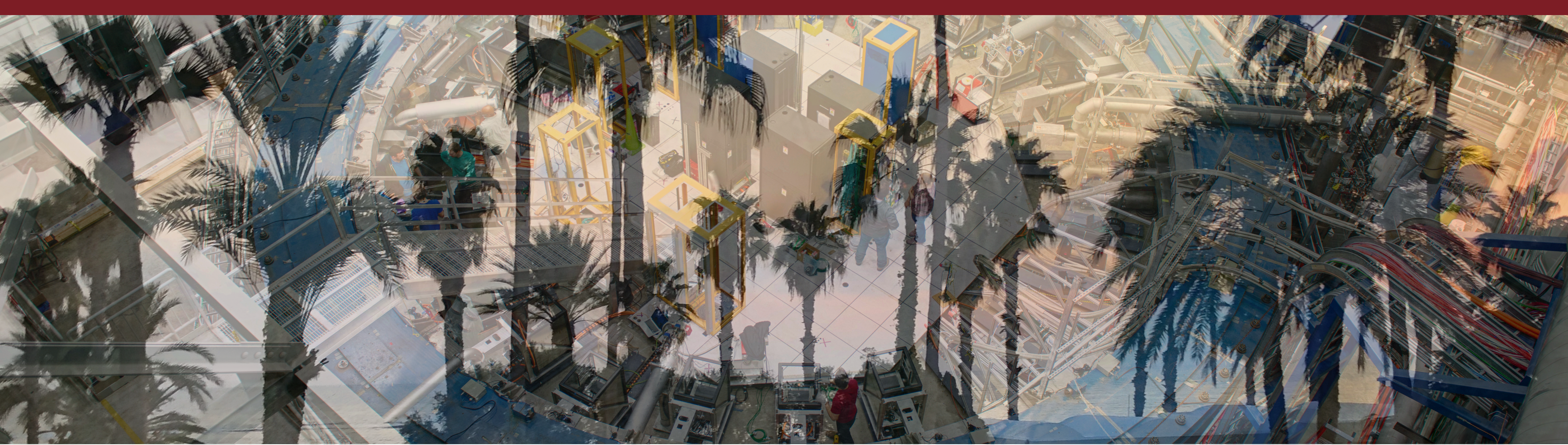

\title{
Measuring the Muon Anomalous Magnetic Moment to High Precision
}

\section{David Flay}

Electromagnetic Interactions with Nucleons and Nuclei, Paphos, Cyprus 31 October 2019 


\section{Outline}

\section{Introduction}

- The Magnetic Moment and the Anomaly

- Recent Theoretical Efforts

\section{The Muon g-2 Experiment at Fermilab}

- Experimental Technique

- Overview of Operations to Date

- Analysis Status

Summary 


\section{The Magnetic Moment and the Anomaly}

\section{The Magnetic Moment}

$\vec{\mu}=g \frac{q}{2 m} \vec{s}$

- Magnetic moment connected to spin via dimensionless g-factor

- Dirac: $g=2$ for $s=1 / 2$ particles (1928)

- Hyperfine structure experiments on hydrogen: $g \neq 2$ (Nafe, Nelson,

Rabi 1947)

- Anomalous contribution $a \equiv(g-2) / 2=a / 2 \pi$ (Schwinger, QED, 1948)

- Radiative corrections from virtual particles in loops 


\section{The Magnetic Moment and the Anomaly}

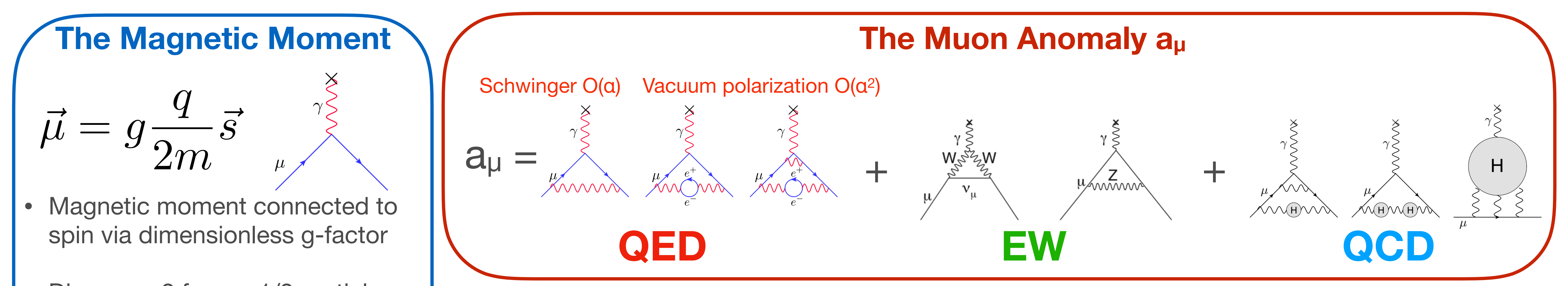

- Dirac: $g=2$ for $s=1 / 2$ particles (1928)

- Hyperfine structure experiments on hydrogen: $g \neq 2$ (Nafe, Nelson, Rabi 1947)

- Anomalous contribution $a \equiv(g-2) / 2=a / 2 \pi$ (Schwinger, QED, 1948)

- Radiative corrections from virtual particles in loops 


\section{The Magnetic Moment and the Anomaly}

\section{Lattice groups making excellent progress (HVP LO, NLO, HLbL)}

\section{Calculation of the hadronic vacuum polarization contribution to the muon}

\section{anomalous magnetic moment}

T. Blum, P.A. Boyle, V. Gülpers, T. Izubuchi, L. Jin, C. Jung, A. Jüttner, C. Lehner, A. Portelli, J.T. Tsang

(Submitted on 22 Jan 2018)

We present a first-principles lattice QCD+QED calculation at physical pion mass of the leading-order hadronic vacuum polarization

contribution to the muon anomalous magnetic moment. The total contribution of up, down, strange, and charm quarks including QED and

strong isospin breaking effects is found to be $a_{\mu}^{\mathrm{HVPLO}}=715.4(16.3)(9.2) \times 10^{-10}$, where the first error is statistical and the second is

systematic. By supplementing lattice data for very s'

we significantly improve the precision of our calculi Higher-order hadronic-vacuum-polarization contribution to the

systematic, R-ratio statistical, and R-ratio systemat
leading-order hadronic vacuum polarization contril

calculation of the light-quark QED correction at ph

B. Chakraborty, C. T. H. Davies, J. Koponen, G. P. Lepage, and R. S. Van de Water (Fermilab Lattice, HPQCD, and

Comments: 12 pages, 11 figures

Subjects: High Energy Physics - Lattice (hep-lat); Hil

arXiv:1801.07224 [hep-lat]

(or arXiv:1801.07224v1 [hep-lat] for this ve

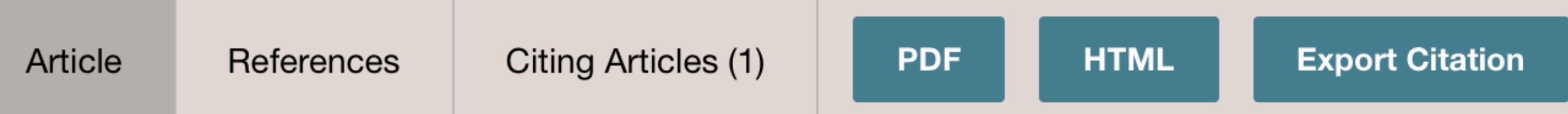

\section{$>$}

\section{ABSTRACT}

We introduce a new method for calculating the $\mathrm{O}\left(\alpha^{3}\right)$ hadronic-vacuum-polarization contribution to

the muon anomalous magnetic moment from ab initio lattice QCD. We first derive expressions suitable

for computing the higher-order contributions either from the renormalized vacuum polarization

function $\hat{\Pi}\left(q^{2}\right)$ or directly from the lattice vector-current correlator in Euclidean space. We then

demonstrate the approach using previously published results for the Taylor coefficients of $\hat{\Pi}\left(q^{2}\right)$ that

virtual particies ill ioups 


\section{The Magnetic Moment and the Anomaly}

\section{Lattice groups making excellent progress (HVP LO, NLO, HLbL)}

\section{Calculation of the hadronic vacuum polariza} anomalous magnetic moment

T. Blum, P.A. Boyle, v. Gülpers, T. Izubuchi, L. Jin, C. Jung, A. Jüttner, C (Submitted on 22 Jan 2018)

We present a first-principles lattice QCD+QED calculation at physical pion ma contribution to the muon anomalous magnetic moment. The total contributio strong isospin breaking effects is found to be $a_{\mu}^{\mathrm{HVP} L O}=715.4(16.3)(9.2) \times 1$ systematic. By supplementing lattice data for very s'

we significantly improve the precision of our calculi Higher-Order

systematic, R-ratio statistical, and R-ratio systemat
leading-order hadronic vacuum polarization contril calculation of the light-quark QED correction at ph

Comments: 12 pages, 11 figures

B. Chakraborty, C. T. $\vdash$ Mhe Collaborations)

Subjects: High Energy Physics - Lattice (hep-lat): Hi arXiv:1801.07224 [hep-lat]

(or arXiv:1801.07224v1 [hep-lat] for this

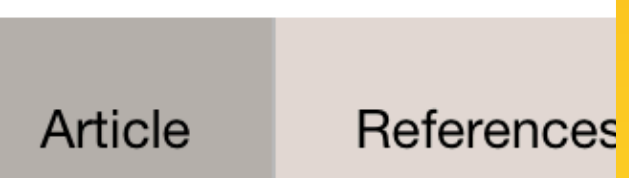

\section{$>$}

virtual particles ill loups
ABS

We in

the $\mathrm{m}$

for cor

function $\hat{\Pi}\left(q^{2}\right)$ or directly from the lattice vector-current correlator in Euclidean space. We then

demonstrate the approach using previously published results for the Taylor coefficients of $\hat{\Pi}\left(q^{2}\right)$ that

\section{2nd g-2 Theory Initiative Meeting in June 2018}

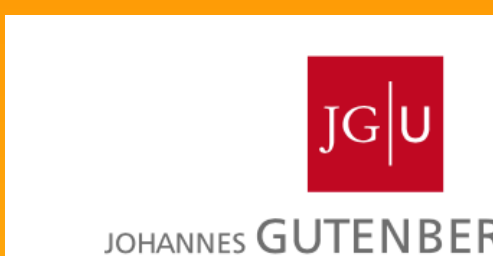

JOHANNES GUTENBERG
UNIVERSITÄT MANZ

Home

Research

People

Publications

Teaching

Events

Talks and Guest Speakers

Previous Events

g-2 Workshop

Press and Media

Services

Contact

(1) home RESEARCh PEOPLE pUblications

Second Plenary Workshop of the Muon g-2 Theory Initiative

18 June 2018 - 22 June 2018

In the coming years, experiments at Fermilab and at J-PARC plan to reduce the uncertainties on the already
very precisely measured anomalous magnetic moment of the muon by a factor of four. The goal is to resolve the current tantalizing tension between theory and experiment of three to four standard deviations. On the theory side the hadronic corrections to the anomalous magnetic moment are the dominant sources of
uncertainty They mus bo uncertainty. They must be determined with better
not new physics effects contribute to this quantity.

There are a number of complementary theoretical efforts underway to better understand and quantify the
hadronic corrections, including dispersive methods, lattice QCD, effective field theories, and QCD models. The Muon $(g-2)$ Theory Initiative was formed in order to facilitate interactions between the different groups
throumb through organizngi a
different communities to discuss, assess, and compare the status of the various efforts and to map out strategies for obtaining the best theoeretical predictions for these hadronic corrections in advance of the
and experimental results.

Dates

Dates

Location
June 18, 2018 - June 22, 2018

GMT+2

Helmholtz-Institut Mainz
Staudinger Weg 18, 55128 Mainz, Ground Floor

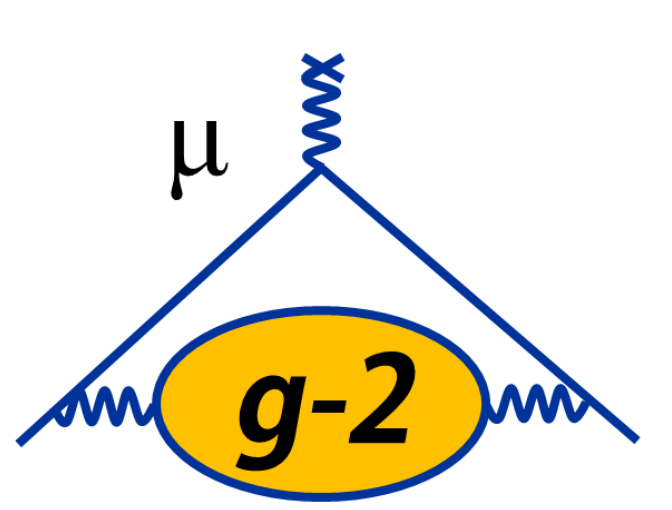




\section{The Magnetic Moment and the Anomaly}

\section{Lattice groups making excellent progress (HVP LO, NLO, HLbL)}

\section{Calculation of the hadronic vacuum polariza} anomalous magnetic moment

$\bar{l}$

T. Blum, P.A. Boyle, V. Gülpers, T. Izubuchi, L. Jin, C. Jung, A. Jüttner, C (Submitted on 22 Jan 2018)

We present a first-principles lattice QCD+QED calculation at physical pion ma contribution to the muon anomalous magnetic moment. The total contributio strong isospin breaking effects is found to be $a_{\mu}^{\mathrm{HVPLO}}=715.4(16.3)(9.2) \times 1$ systematic. By supplementing lattice data for very s

we significantly improve the precision of our calculi Higher-order

systematic, R-ratio statistical, and R-ratio systemat
leading-order hadronic vacuum polarization contril

calculation of the light-quark QED correction at ph.

Comments: 12 pages, 11 figures

B. Chakraborty, C. T. H

Phys. Rev. 98,0945

Subjects: High Energy Physics - Lattice (hep-lat); Hit

arXiv:1801.07224 [hep-lat]

(or arXiv:1801.07224v1 [hep-lat] for this
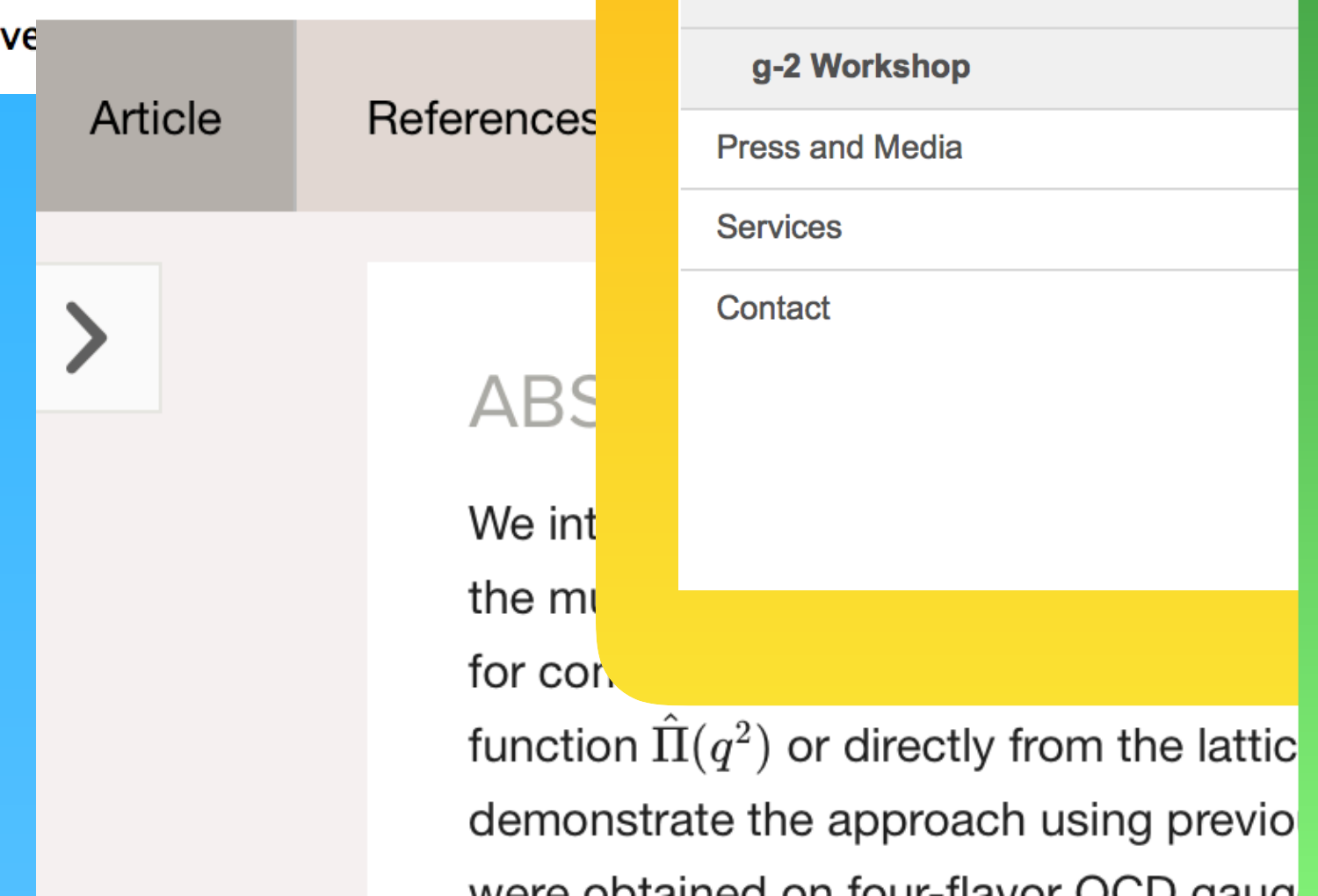

virtual particles III luops

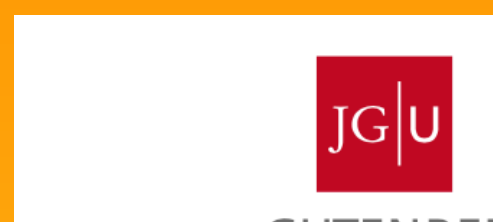

JOHANNES GUTENBERG
UNIVERSITÄT MANZ

Home

Research

People

Publications

Teaching

Events

Talks and Guest Speakers

Previous Events

g-2 Workshop

Press and Media

Services

Contact

\section{2nd g-2 Theory Initiative Meeting in June 2018}

\section{3rd g-2 Theory Initiative Meeting in Sept 2019}

\section{INT Workshop INT-19-74W}

Hadronic contributions to $(\mathrm{g}-2)_{\mu}$

September 9 - 13, 2019
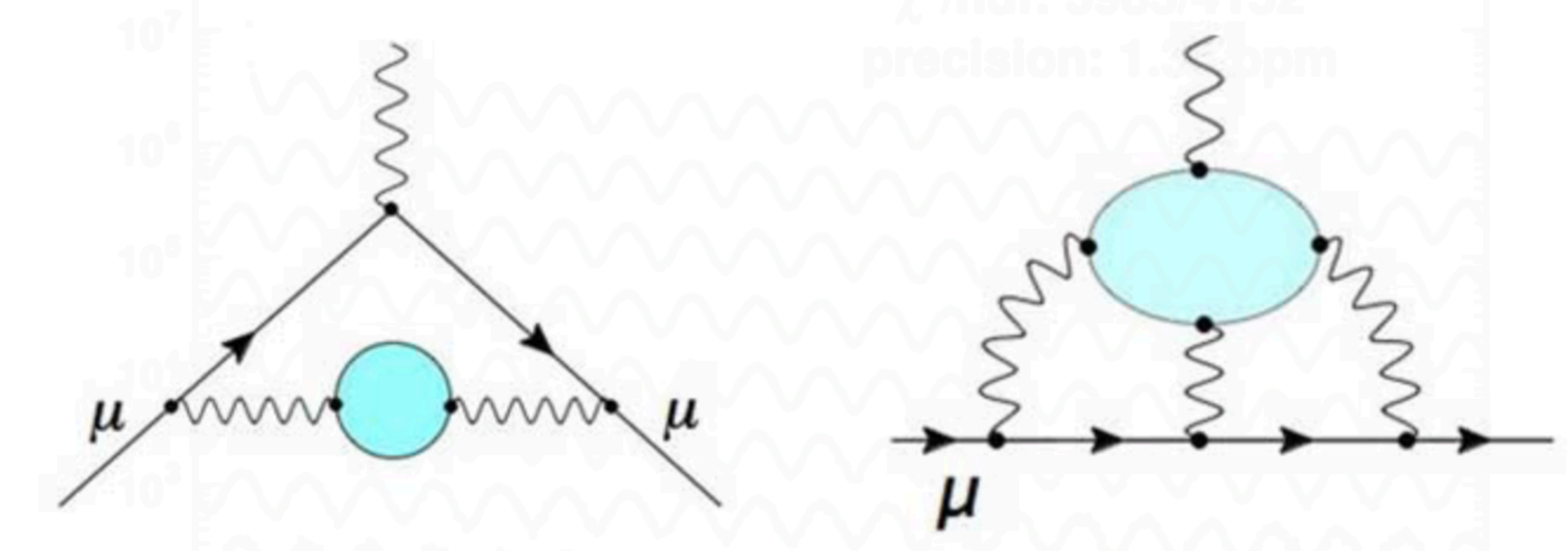

8 David Flay I Measuring the Muon Anomalous Magnetic Moment to High Precision 


\section{$a_{\mu}$ Theoretical Status}

\begin{tabular}{|c|c|c|}
\hline Contribution & Value (x 10-11) & Reference \\
\hline QED & $116584718.95 \pm 0.08$ & PRL 109 111808 (2012) \\
\hline EW & $153.6 \pm 1.0$ & PRD 88 053005 (2013) \\
\hline & & \\
\hline & & \\
\hline & & \\
\hline & & \\
\hline & & \multicolumn{2}{|c|}{} \\
\hline & & \\
\hline & \multicolumn{3}{|c|}{} \\
\hline
\end{tabular}




\section{$a_{\mu}$ Theoretical Status}

\begin{tabular}{|c|c|c|}
\hline \multirow{2}{*}{$\begin{array}{c}\text { Contribution } \\
\text { QED }\end{array} 116584718.95 \pm 0.08$} & PRL 109 111808 (2012) \\
\hline EW & $153.6 \pm 1.0$ & PRD 88 053005 (2013) \\
\hline HVP (LO) & $6931 \pm 34$ & EPJ C 77 827 (2017) \\
\hline HVP (LO) & $\mathbf{6 9 3 3 \pm \mathbf { 2 5 }}$ & PRD 97 114025 (2018) \\
\hline & & \\
\hline & & \\
\hline & & \\
\hline & & \\
\hline & & \\
& & \\
\hline
\end{tabular}

HVP (LO): Lowest-Order Hadronic Vacuum Polarization

- Critical input from e+e- colliders (data from SND, CMD3, BaBar, KLOE, Belle, BESIII), extensive physics program running to reduce $\delta \mathrm{a}_{\mu} \mathrm{HVP}$ to $\sim 0.3 \%$ in coming years

- Progress on the lattice: Calculations at physical $\pi$ mass; approaching goal of $\delta a_{\mu} \mathrm{HVP} \sim 1 \%$ (cross-check with e+e- data)

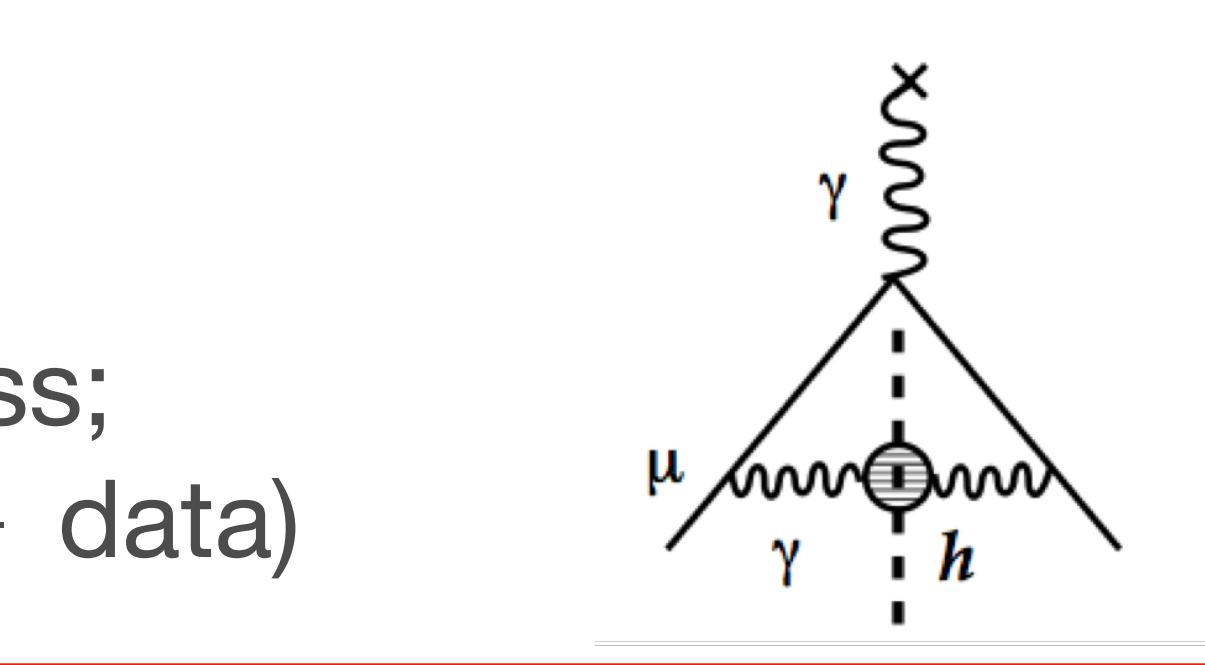

$a_{\mu}^{\mathrm{had} ; \mathrm{LO}}=\left(\frac{\alpha m_{\mu}}{3 \pi}\right)^{2} \int_{m_{\pi}^{2}}^{\infty} \frac{d s}{s^{2}} K(s) R(s)$ $R \equiv \frac{\sigma_{\text {tot }}\left(e^{+} e^{-} \rightarrow \text { hadrons }\right)}{\sigma\left(e^{+} e^{-} \rightarrow \mu^{+} \mu^{-}\right)}$

10 David Flay I Measuring the Muon Anomalous Magnetic Moment to High Precision

UMassAmherst 


\section{$a_{\mu}$ Theoretical Status}

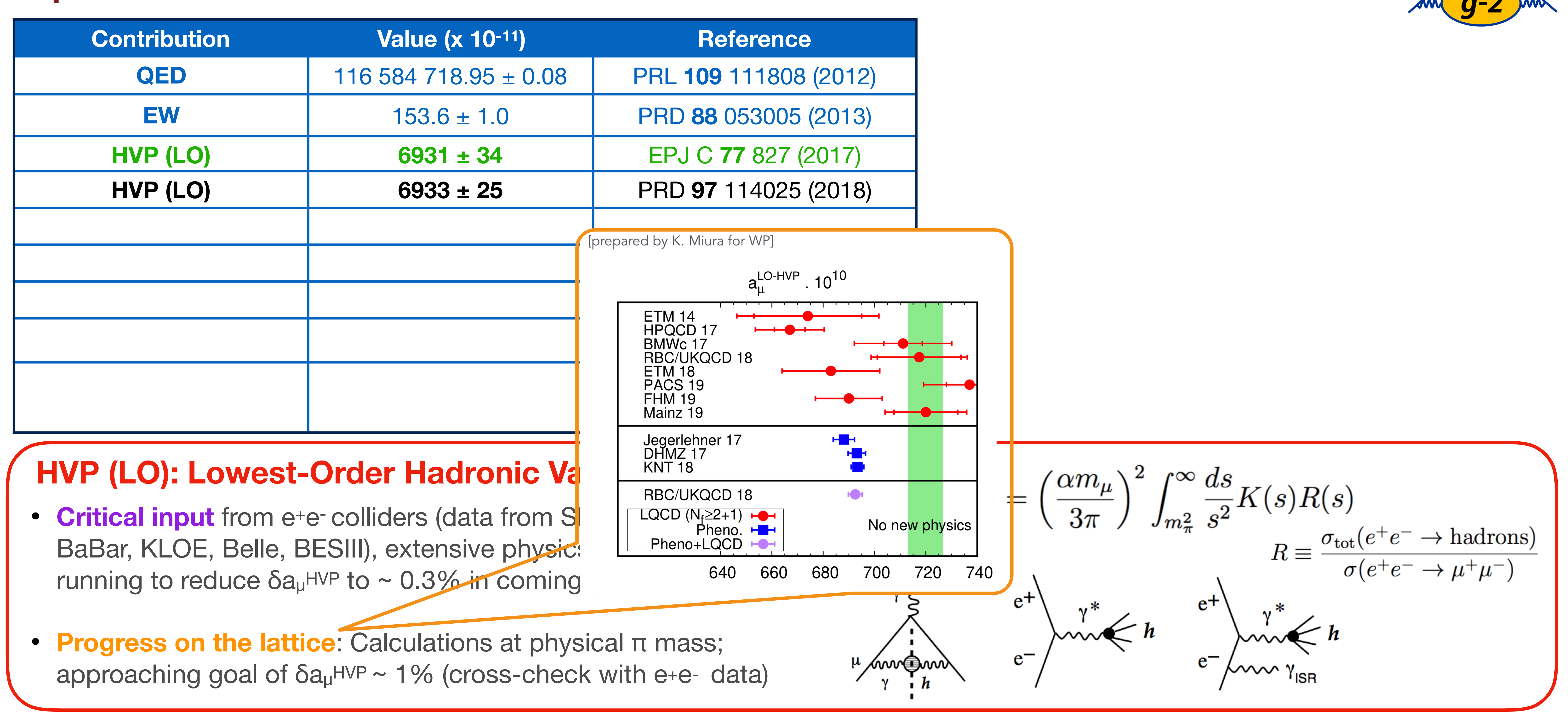

11 David Flay I Measuring the Muon Anomalous Magnetic Moment to High Precision 


\section{$a_{\mu}$ Theoretical Status}

\begin{tabular}{|c|c|c|}
\hline Contribution & Value (x 10-11) & Reference \\
\hline QED & $116584718.95 \pm 0.08$ & PRL 109 111808 (2012) \\
\hline EW & $153.6 \pm 1.0$ & PRD 88 053005 (2013) \\
\hline HVP (LO) & $6931 \pm 34$ & EPJ C 77 $827(2017)$ \\
\hline HVP (LO) & $\mathbf{6 9 3 3 \pm 2 5}$ & PRD 97 114025 (2018) \\
\hline HVP (NLO) & $\mathbf{- 9 8 . 7 \pm 0 . 7}$ & EPJ C 77 827 (2017) \\
\hline HVP (NLO) & $\mathbf{- 9 8 . 2 \pm 0 . 4}$ & PRD 97 114025 (2018) \\
\hline HVP (NNLO) & $12.4 \pm 0.1$ & PLB 734 144 (2014) \\
\hline & & \\
\hline & & \\
\hline
\end{tabular}

HVP (LO): Lowest-Order Hadronic Vacuum Polarization

- Critical input from $e^{+} e^{-}$colliders (data from SND, CMD3, BaBar, KLOE, Belle, BESIII), extensive physics program running to reduce $\delta \mathrm{a}_{\mu} \mathrm{HVP}$ to $\sim 0.3 \%$ in coming years

- Progress on the lattice: Calculations at physical $\pi$ mass; approaching goal of $\delta a_{\mu} H V P \sim 1 \%$ (cross-check with e+e- data)

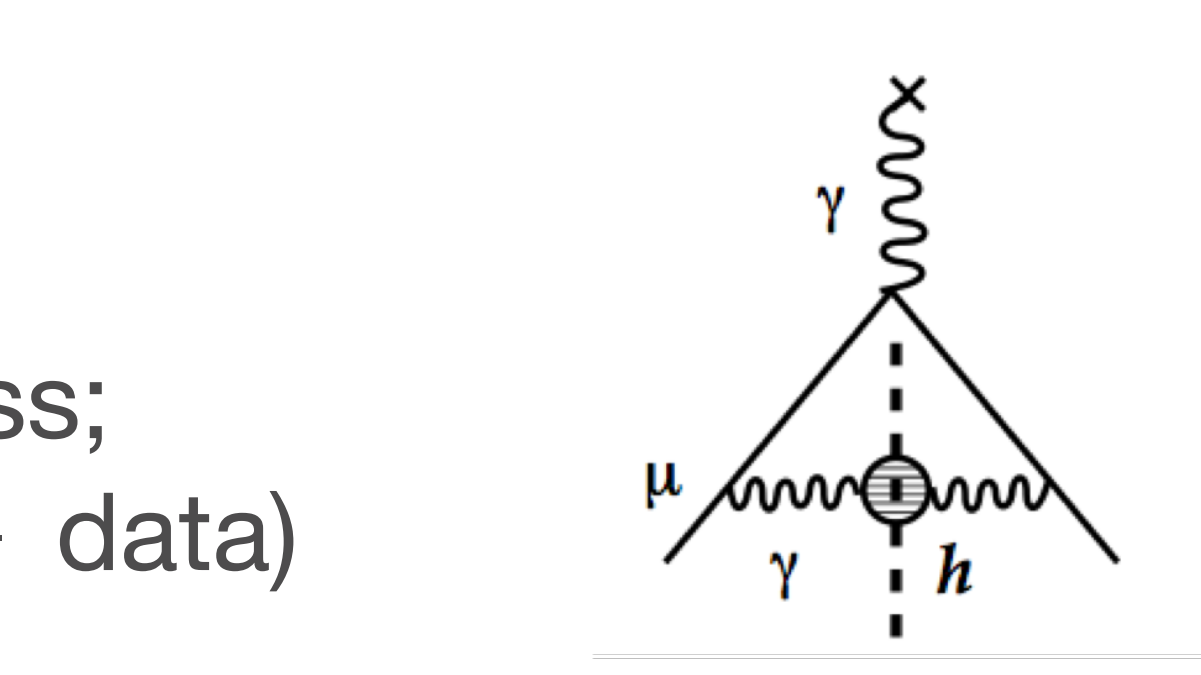
$a_{\mu}^{\mathrm{had} ; \mathrm{LO}}=\left(\frac{\alpha m_{\mu}}{3 \pi}\right)^{2} \int_{m_{\pi}^{2}}^{\infty} \frac{d s}{s^{2}} K(s) R(s)$ $R \equiv \frac{\sigma_{\text {tot }}\left(e^{+} e^{-} \rightarrow \text { hadrons }\right)}{\sigma\left(e^{+} e^{-} \rightarrow \mu^{+} \mu^{-}\right)}$

12 David Flay I Measuring the Muon Anomalous Magnetic Moment to High Precision 


\title{
$a_{\mu}$ Theoretical Status
}

New ab initio approaches [PRD 98094503 (2018)]

finding consistent result of $(-93 \pm 13) \times 10^{-11}-$

112)

lattice making big strides

j18)

\begin{tabular}{|c|c|c|}
\hline HV. $、$ NLO) & $-98.7 \pm 0.7$ & EPJ C 77827 (2017) \\
\hline HVP (NLO) & $-98.2 \pm 0.4$ & PRD 97114025 (2018) \\
\hline HVP (NNLO) & $12.4 \pm 0.1$ & PLB 734144 (2014) \\
\hline
\end{tabular}

HVP (LO): Lowest-Order Hadronic Vacuum Polarization

- Critical input from e+e- colliders (data from SND, CMD3, BaBar, KLOE, Belle, BESIII), extensive physics program running to reduce $\delta a_{\mu} \mathrm{HVP}$ to $\sim 0.3 \%$ in coming years

- Progress on the lattice: Calculations at physical $\pi$ mass; approaching goal of $\delta a_{\mu} H V P \sim 1 \%$ (cross-check with e+e- data)

\author{
data)
}

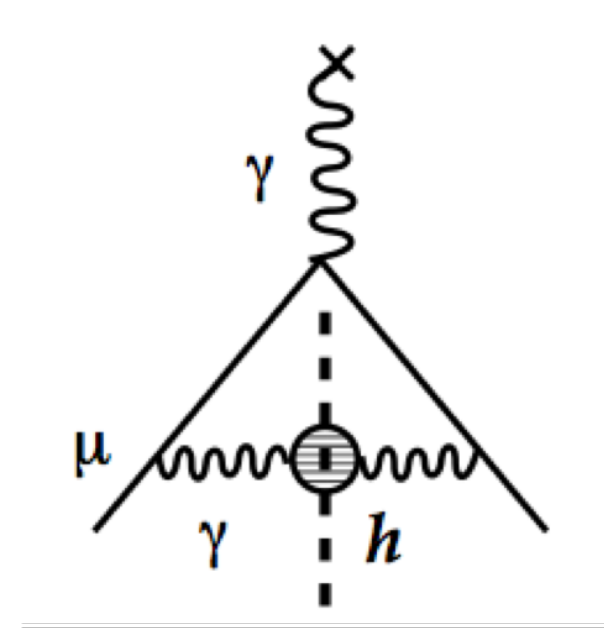

$a_{\mu}^{\mathrm{had} ; \mathrm{LO}}=\left(\frac{\alpha m_{\mu}}{3 \pi}\right)^{2} \int_{m_{\pi}^{2}}^{\infty} \frac{d s}{s^{2}} K(s) R(s)$ $R \equiv \frac{\sigma_{\text {tot }}\left(e^{+} e^{-} \rightarrow \text { hadrons }\right)}{\sigma\left(e^{+} e^{-} \rightarrow \mu^{+} \mu^{-}\right)}$

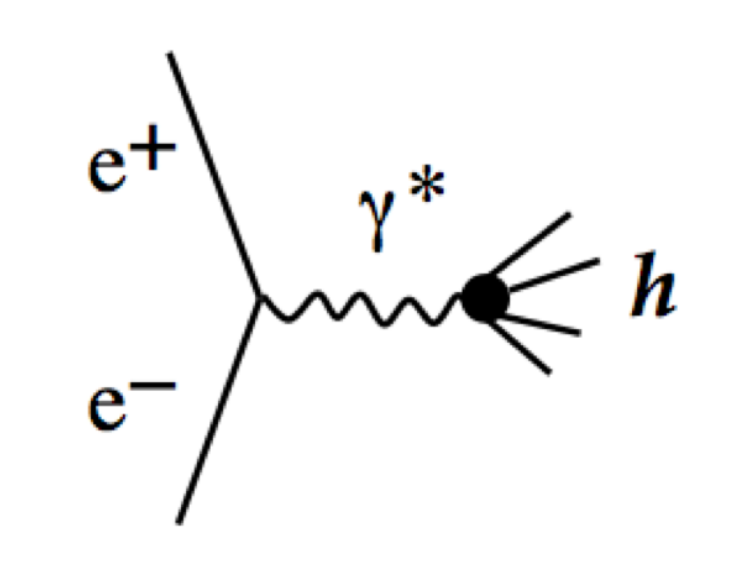

$\mathrm{e}^{+} \operatorname{e}^{-} \gamma^{*} \gamma_{\text {ISR }}{ }_{h}$ 


\section{$a_{\mu}$ Theoretical Status}

New ab initio approaches [PRD 98094503 (2018)]

finding consistent result of $(-93 \pm 13) \times 10^{-11}-$

lattice making big strides

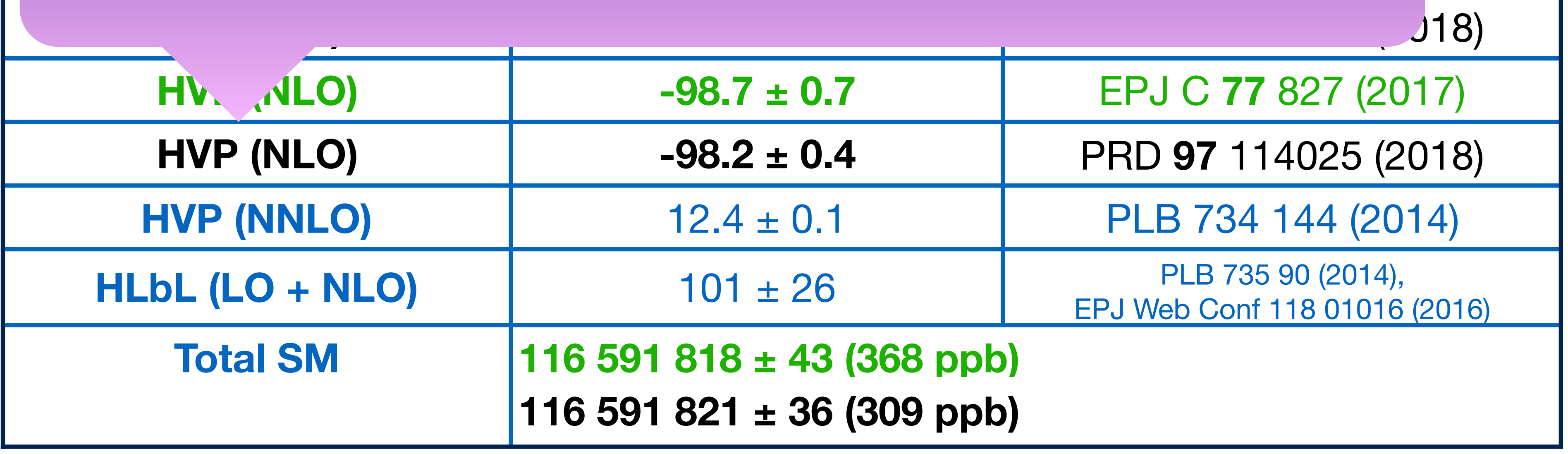

HLbL: Hadronic Light-by-Light

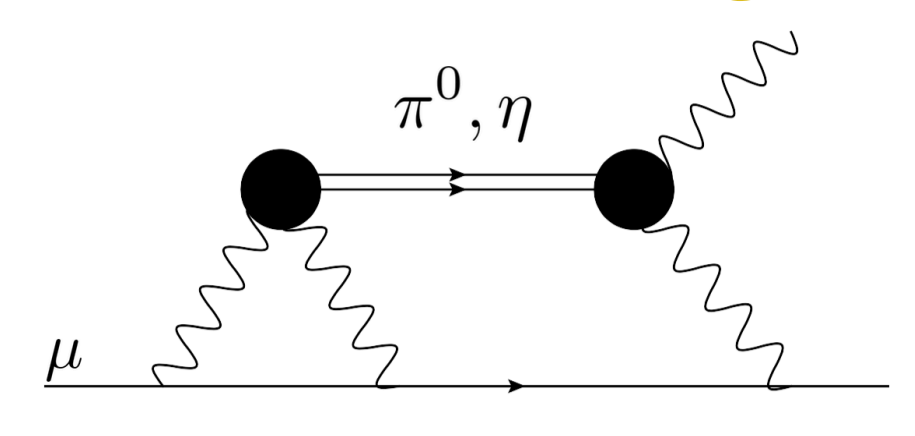

- Model dependent: based on XPT + short-distance constraints (operator product expansion)

- Difficult to relate to data like HVP (LO); $\gamma^{\star}$ physics, $\pi^{0}$ data (BESIII, KLOE) important for constraining models

- Theory Progress: New dispersive calculation approach; extend the lattice (finite volume, disconnected diagrams); Blum et al. making excellent progress

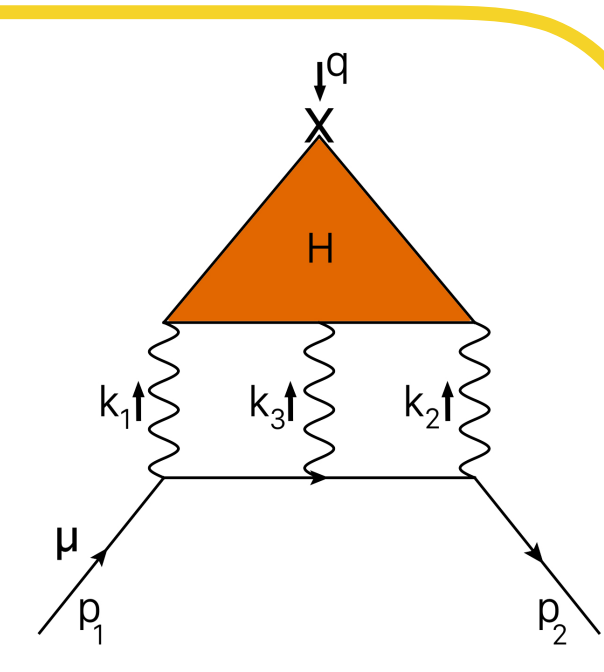

\section{HVP (LO): Lowest-Order Hadronic Vacuum Polarization}

- Critical input from $e^{+} e^{-}$colliders (data from SND, CMD3, BaBar, KLOE, Belle, BESIII), extensive physics program running to reduce $\delta \mathrm{a}_{\mu} \mathrm{HVP}$ to $\sim 0.3 \%$ in coming years

- Progress on the lattice: Calculations at physical $\pi$ mass; approaching goal of $\delta a_{\mu} H V P \sim 1 \%$ (cross-check with e+e- data)

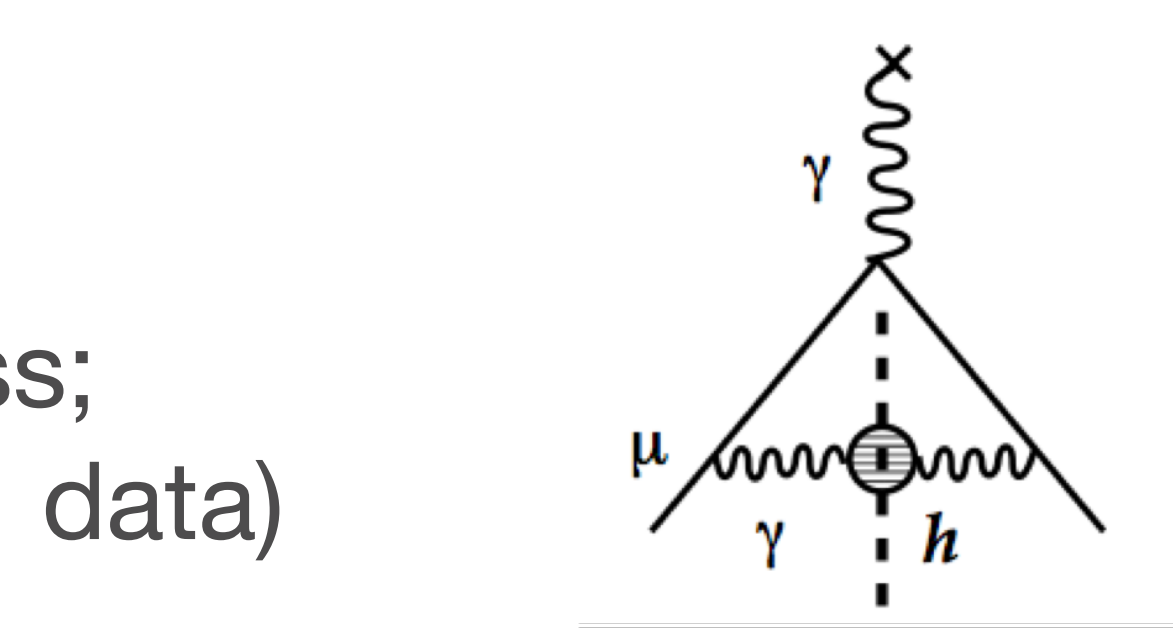

$a_{\mu}^{\mathrm{had} ; \mathrm{LO}}=\left(\frac{\alpha m_{\mu}}{3 \pi}\right)^{2} \int_{m_{\pi}^{2}}^{\infty} \frac{d s}{s^{2}} K(s) R(s)$ $R \equiv \frac{\sigma_{\text {tot }}\left(e^{+} e^{-} \rightarrow \text { hadrons }\right)}{\sigma\left(e^{+} e^{-} \rightarrow \mu^{+} \mu^{-}\right)}$

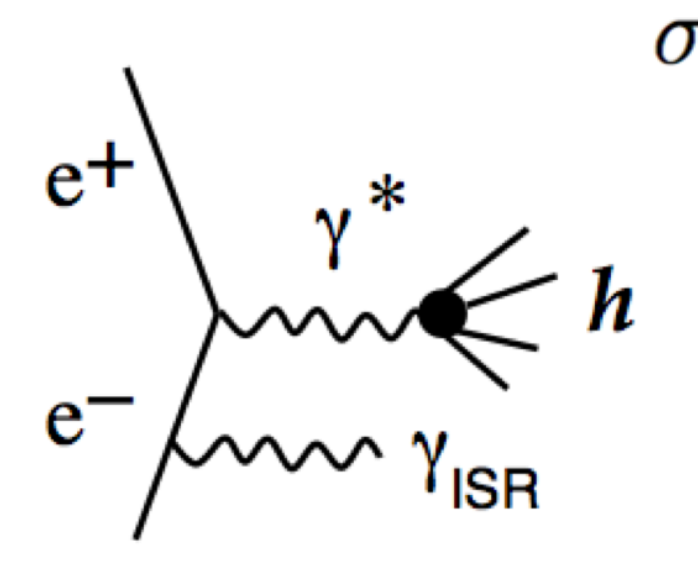

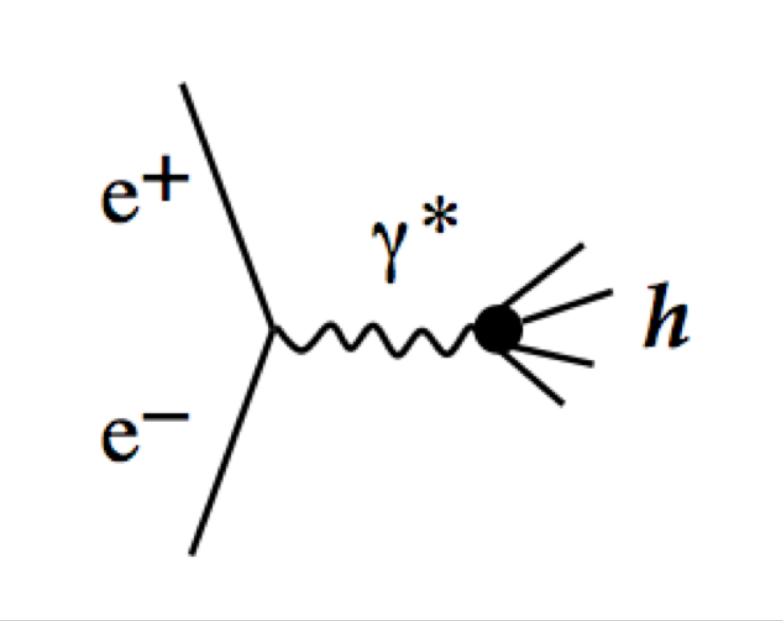




\section{$a_{\mu}$ Theoretical Status}

New ab initio approaches [PRD 98094503 (2018)]

finding consistent result of $(-93 \pm 13) \times 10^{-11}-$ lattice making big strides

\begin{tabular}{|c|c|c|}
\hline HV. $、$ NLO) & $-98.7 \pm 0.7$ & EPJ C 77827 (2017) \\
\hline HVP (NLO) & $-98.2 \pm 0.4$ & PRD 97114025 (2018) \\
\hline HVP (NNLO) & $12.4 \pm 0.1$ & PLB 734144 (2014) \\
\hline HLbL (LO + NLO) & $101 \pm 26$ & $\begin{array}{c}\text { PLB } 73590(2014), \\
\text { EPJ Web Conf } 11801016(2016)\end{array}$ \\
\hline Total SM & \multicolumn{2}{|c|}{$\begin{array}{l}116591818 \pm 43(368 p p b) \\
116591821 \pm 36(309 p p b)\end{array}$} \\
\hline
\end{tabular}

12) 13)

7)

7)

8)

HVP (LO): Lowest-Orde

- Critical input from $\mathrm{e}^{+} \mathrm{e}^{-}$colli BaBar, KLOE, Belle, BESIII), running to reduce $\delta a_{\mu} H V P$ to

- Progress on the lattice: C approaching goal of $\delta a_{\mu} H V P$

Recent lattice \& data-driven estimate [PRD 100034520 (2019)] for $a_{\mu}^{\pi^{0}}$-pole is consistent with lowest-meson dominance, + vector phenomenological models [PRD 514939 (2005), PRL 83 5230 (1999), EJC 21659 (2001), PRD 65073034 (2002), PRD 94053006 (2016), EJC 75586 (2015)]
HLb L: Hadronic Light-by-Light
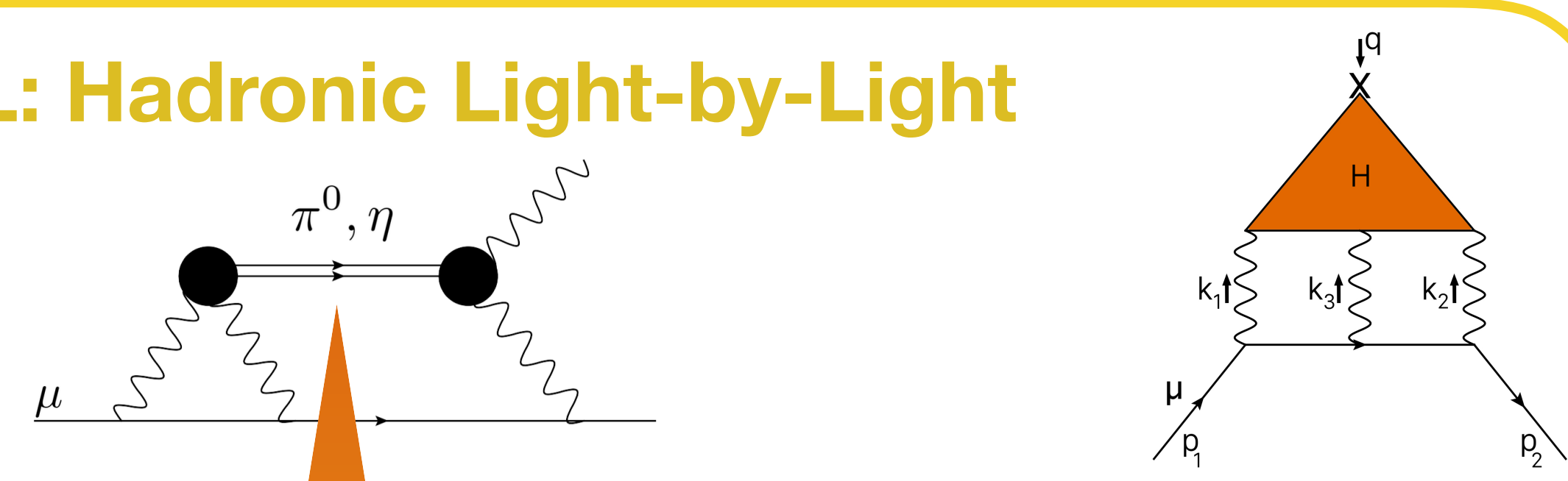

- Model depende constraints (op

- Difficult to rela (BESIII, KLOE

based on XPT + short-distance tor product expansion)

data like HVP (LO); $\gamma^{\star}$ physics, $\Pi^{0}$ data rrtant for constraining models

- Theory Prog extend the I Blum et al. ew dispersive calculation approach; ite volume, disconnected diagrams); rcellent progress $\frac{{ }_{\mathrm{t}}\left(e^{+} e^{-} \rightarrow \text { hadrons }\right)}{\sigma\left(e^{+} e^{-} \rightarrow \mu^{+} \mu^{-}\right)}$$$
\sigma(e+
$$

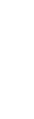




\section{$a_{\mu}$ Theoretical Status}

New ab initio approaches [PRD 98094503 (2018)]

finding consistent result of $(-93 \pm 13) \times 10^{-11}-$ lattice making big strides

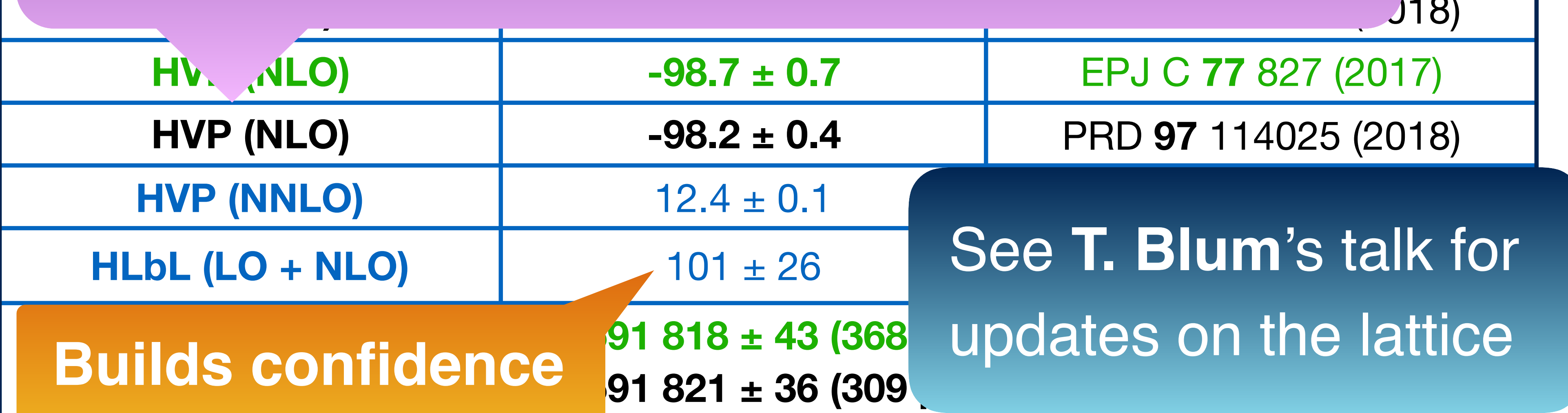
in HLbL term

- Critical input from $\mathrm{e}^{+} \mathrm{e}^{-}$colli BaBar, KLOE, Belle, BESIII), running to reduce $\delta a_{\mu} H V P$ to

- Progress on the lattice: $\mathrm{C}$ c approaching goal of $\delta a_{\mu} H V P$.
Recent lattice \& data-driven estimate [PRD 100034520 (2019)] for $a_{\mu}^{\pi^{0}}$-pole is consistent with lowest-meson dominance, + vector phenomenological models [PRD 514939 (2005), PRL 83 5230 (1999), EJC 21659 (2001), PRD 65073034 (2002), PRD 94053006 (2016), EJC 75586 (2015)] 

progress to achieve competitive uncertainties on the same time scale as the FNAL experiment result -White paper discussing all terms forthcoming

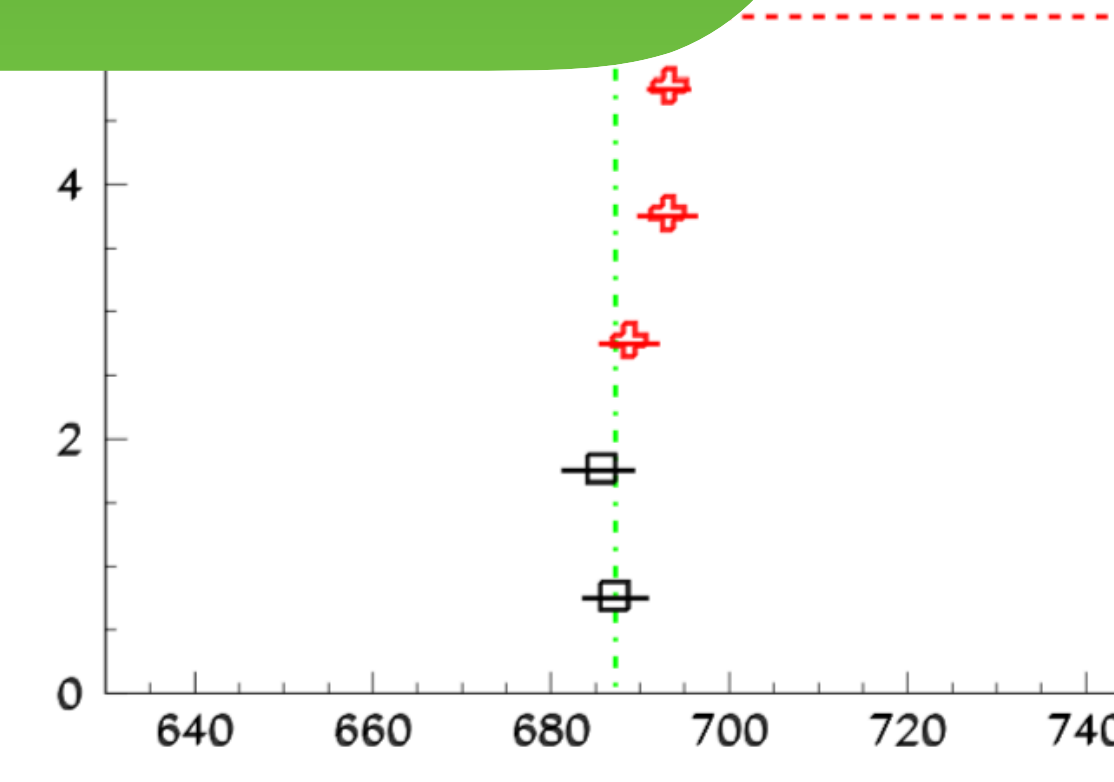

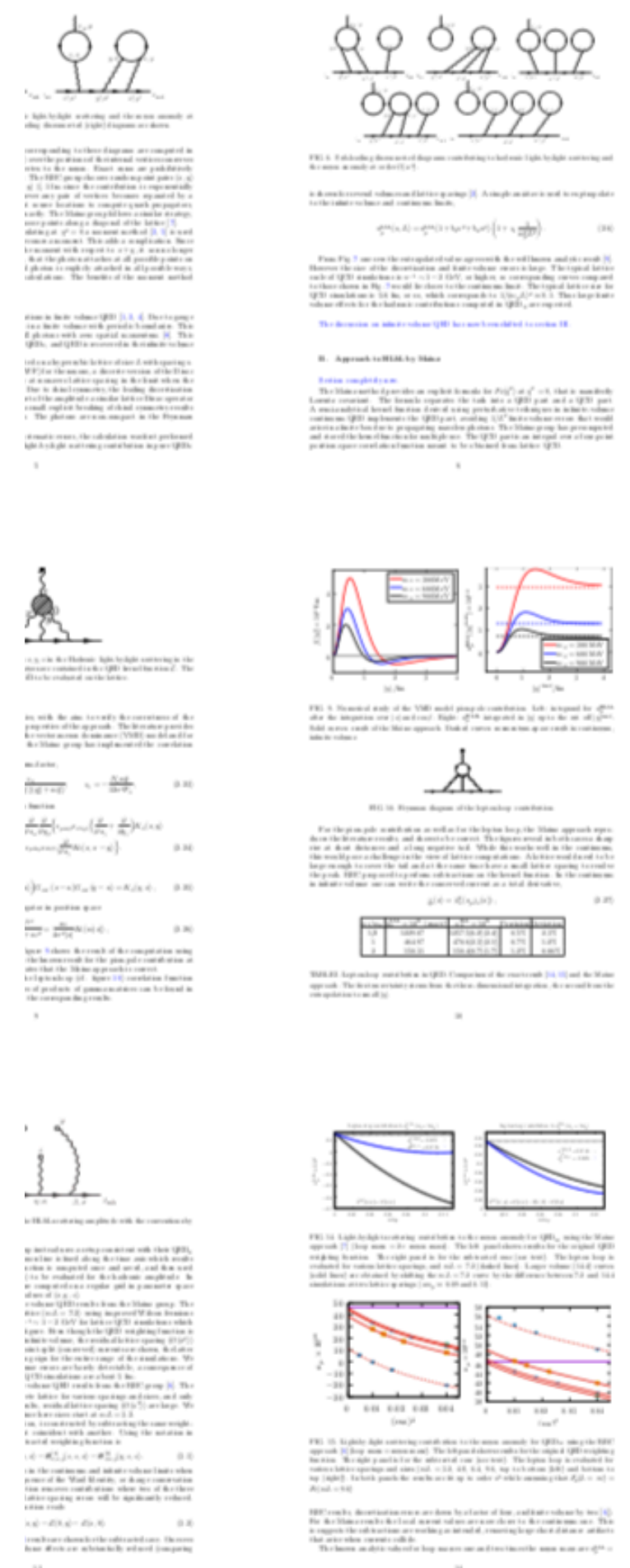

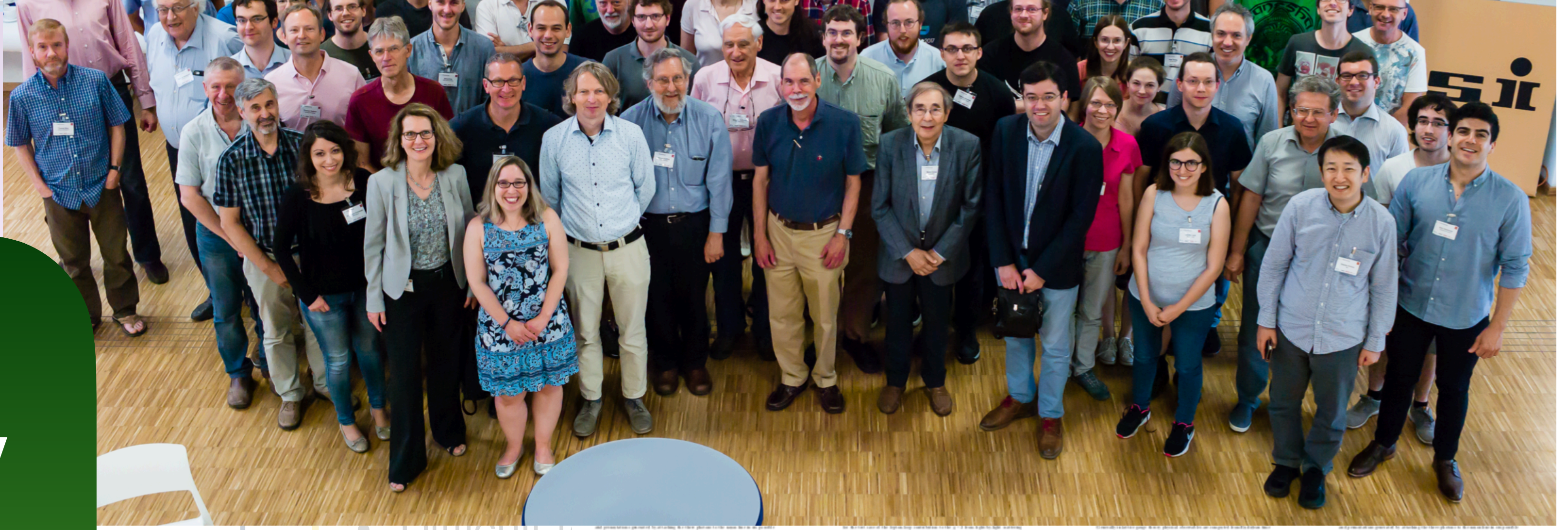

Direct Integration 201
$687.24 \pm 4.19$

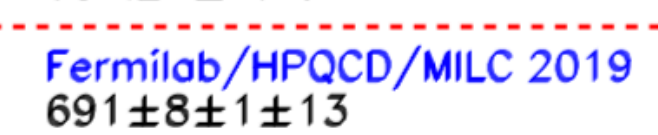

ETMC 2018

$\mathrm{RBC} / \mathrm{NKOCO} 2018$
$715.4 \pm 16.3 \pm 9.2$

BMW 2017
$711.0 \pm 7.5 \pm 17.3$

$\mathrm{HPQCD} / \mathrm{RV} 2016$
$666 \pm 6 \pm 12$

Moinz $/$ CLS 2019
$720.0 \pm 12.4 \pm 9.9$

REC M UKCOC 2018
$692.5 \pm 1.4 \pm 0.5 \pm 0.7 \pm 2.1$

$\underset{693.2612 .46}{\mathrm{KN} 2018}$

DHMZ 2017
$693.1 \pm 3.4$

$\mathrm{F} J 2017$
$688.77 \pm 3.38$

BHIS 2015 ydd 2019
$685.64 \pm 2.91+(+1.26,-1.92)_{\text {mom }}$

$\mathrm{BH} \mathrm{LS}_{2} 2019$
$687.11 \pm 3.01+(+1.26,-1.02)$

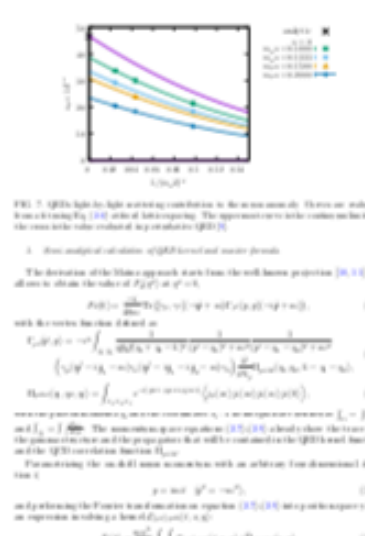

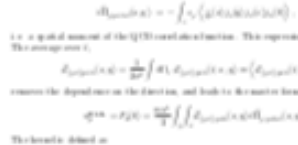

$=$

$+\quad+$

国

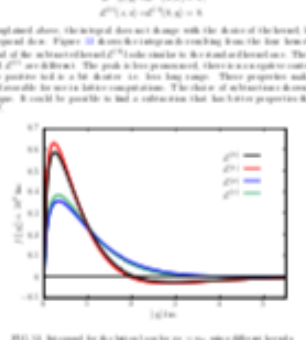

$\frac{15}{2+5}$

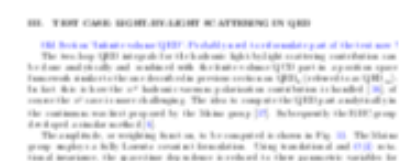

$+$

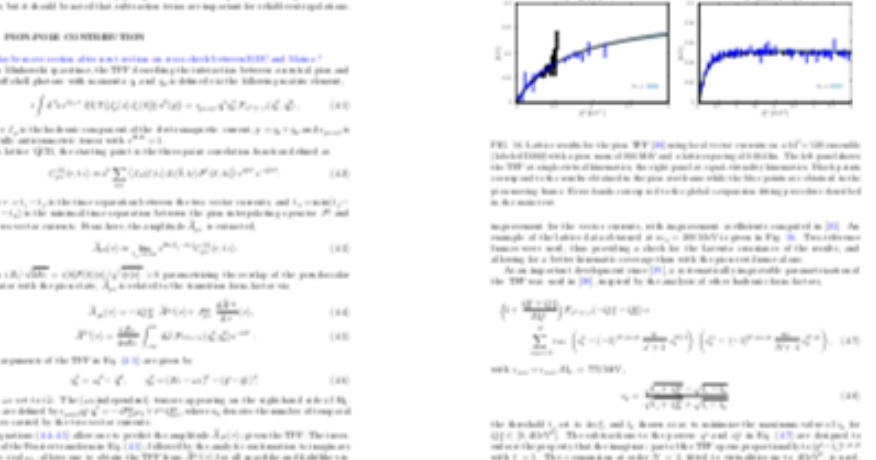




\section{The Muon g-2 Experiment at Fermilab}




\section{Muon g-2: 33 Institutions, 7 countries, 203 Members}
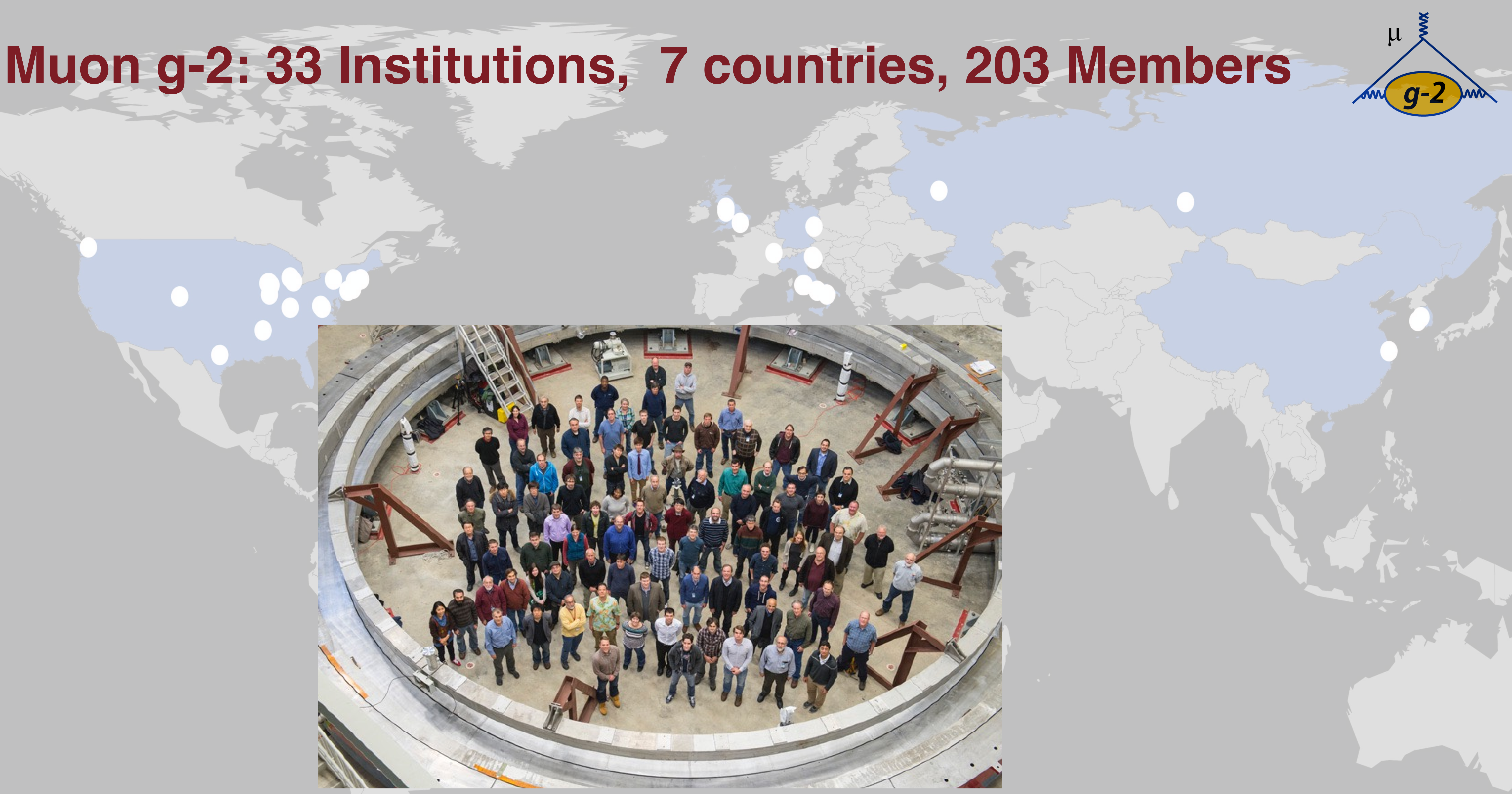

UMassAmherst

19 David Flay I Measuring the Muon Anomalous Magnetic Moment to High Precision 


\section{Why Fermilab?}

- BNL limited by statistics (540 ppb on $9 \times 10^{9}$ detected $\mathrm{e}^{+}$)

- E989 goal: Factor of 21 more statistics $\left(2 \times 10^{11}\right.$ detected $\left.\mathrm{e}^{+}\right)$

\section{Fermilab advantages}

$\checkmark$ Long beam line to collect $\pi^{+} \rightarrow \mu^{+}$

Much reduced amount of $p, \pi$ in ring $\checkmark$ 4x higher fill frequency than BNL
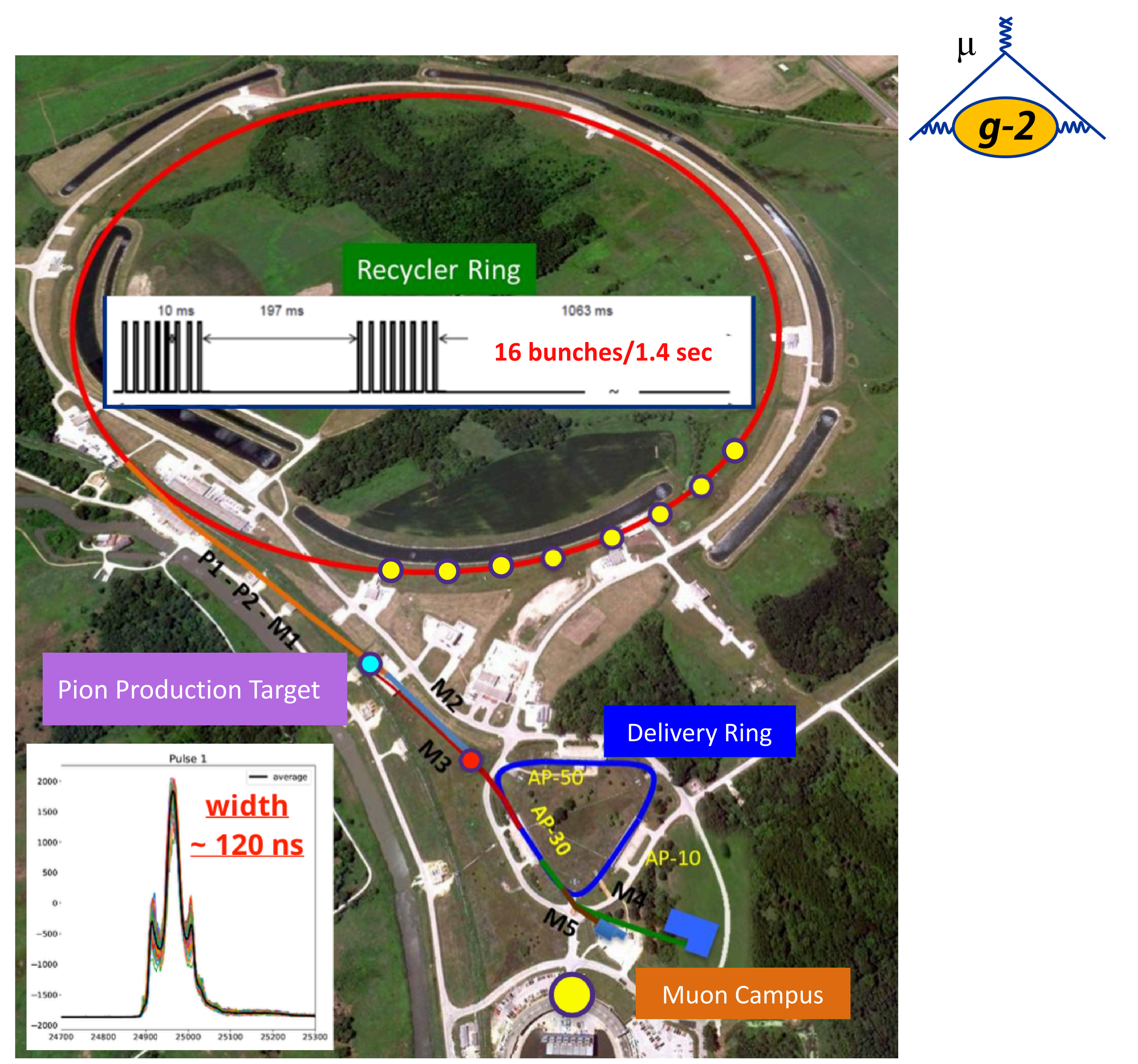


\section{Measuring the Muon Anomaly}

- Inject polarized muon beam into magnetic storage ring

$\vec{\omega}_{C}=-\frac{e}{\gamma m} \vec{B}_{\text {cyclotron frequency }}$

- Measure difference

$\vec{\omega}_{S}=-\frac{e}{\gamma m} \vec{B}\left(1+\gamma a_{\mu}\right)$

spin precession frequency between spin precession and cyclotron frequencies

- If $g=2, \omega_{a}=0$

- $g \neq 2, \omega_{a} \cong\left(e / m_{\mu}\right) a_{\mu} B$

$\vec{\omega}_{a} \equiv \vec{\omega}_{S}-\vec{\omega}_{C}$

- Using $\hbar \omega_{p}=2 \mu_{p}|\vec{B}|$ :

$a_{\mu}=\frac{\omega_{a}}{\tilde{\omega}_{p}} \frac{\mu_{p}}{\mu_{e}} \frac{m_{\mu}}{m_{e}} \frac{g_{e}}{2}$

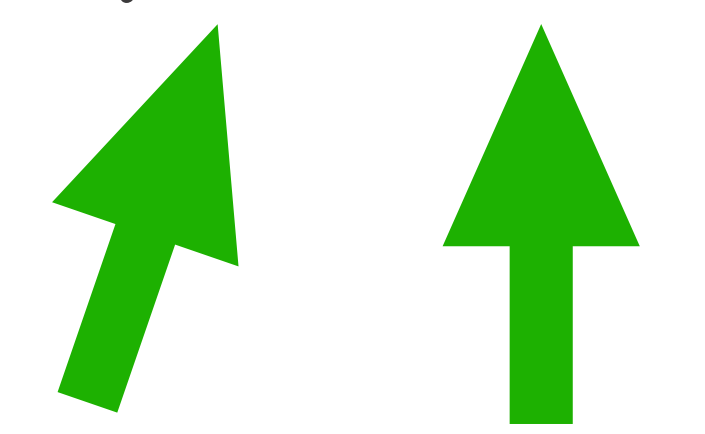

$3 \mathrm{ppb}$

$22 \mathrm{ppb}$

Rev. Mod. Phys. 88, 035009 (2016)
- We measure $\omega_{a}$ and $\omega_{p}$ separately

- Aiming for $70 \mathrm{ppb}$ precision on each (systematic)

- Target: $\delta a_{\mu}=140$ ppb; factor of 4 improvement over BNL

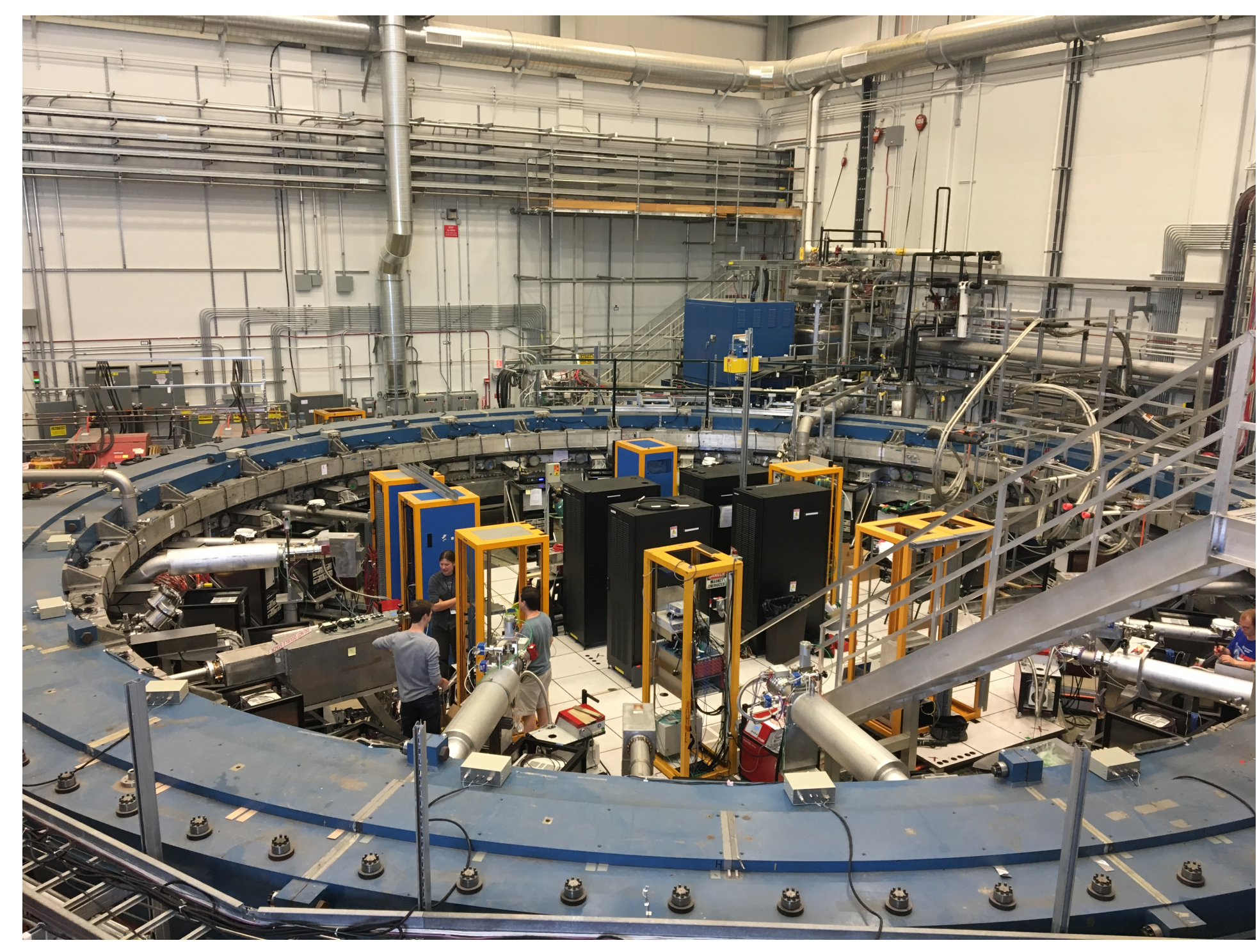

UMassAmherst 


\section{Muon Beam Injection}

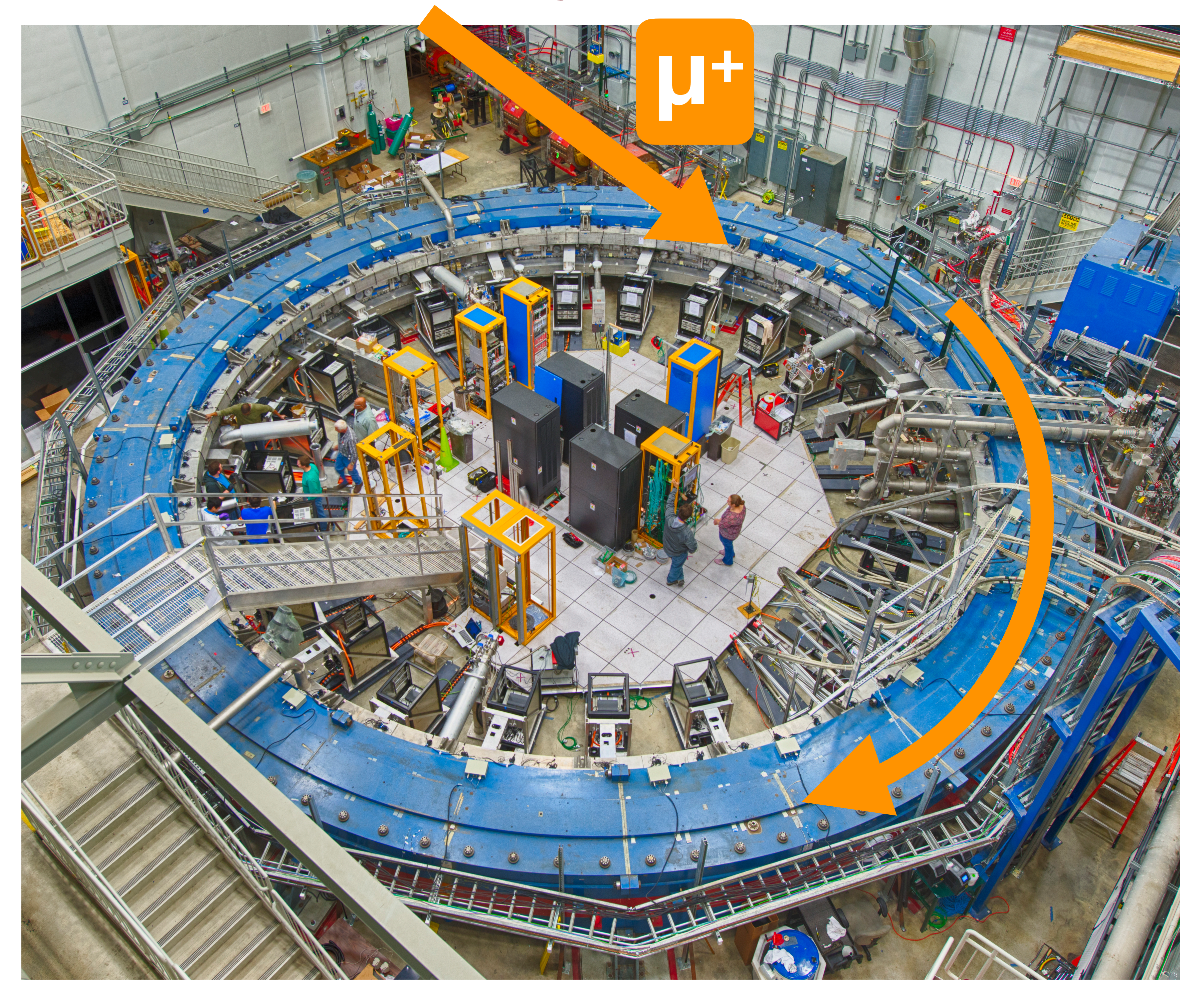




\section{Muon Beam Injection}

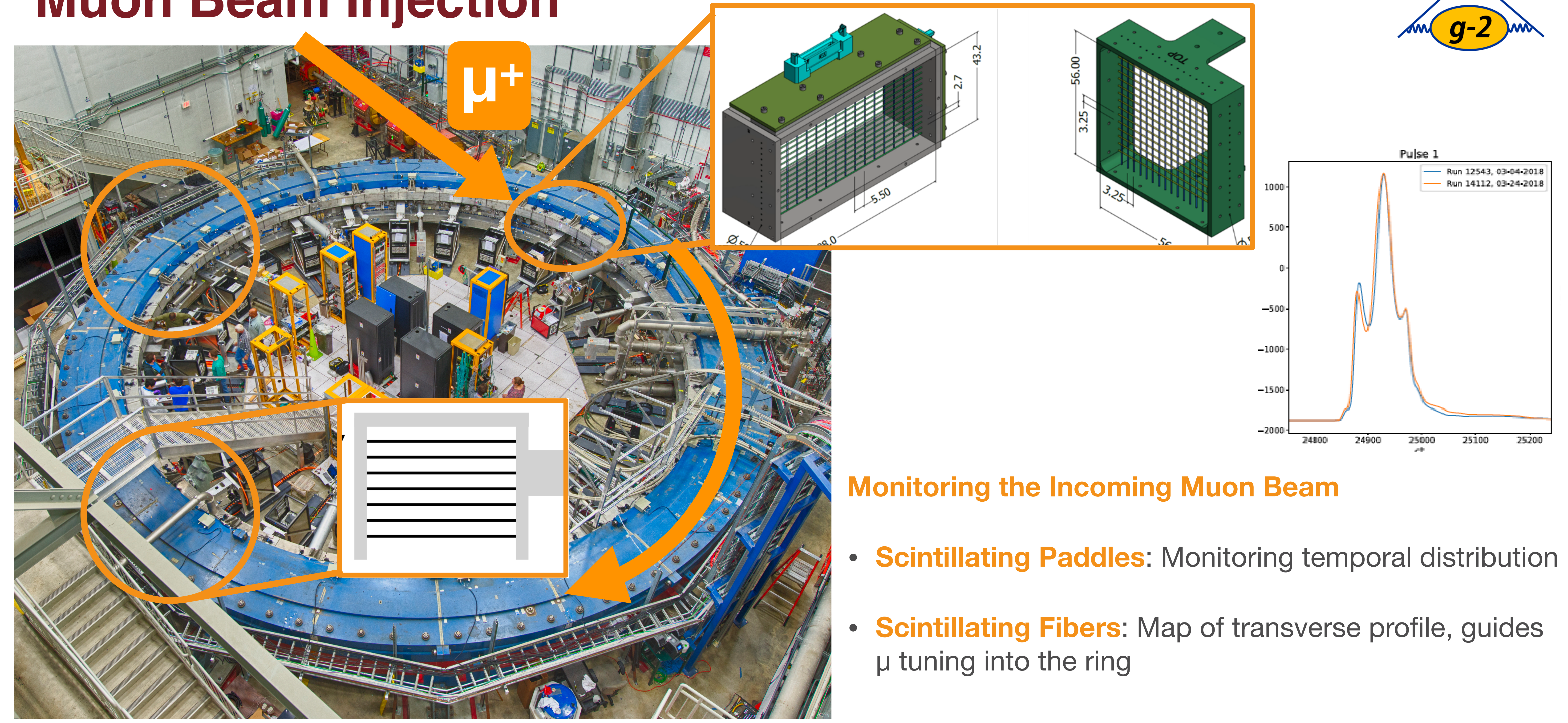

24 David Flay I Measuring the Muon Anomalous Magnetic Moment to High Precision 


\section{Muon Beam Storage and Focusing}
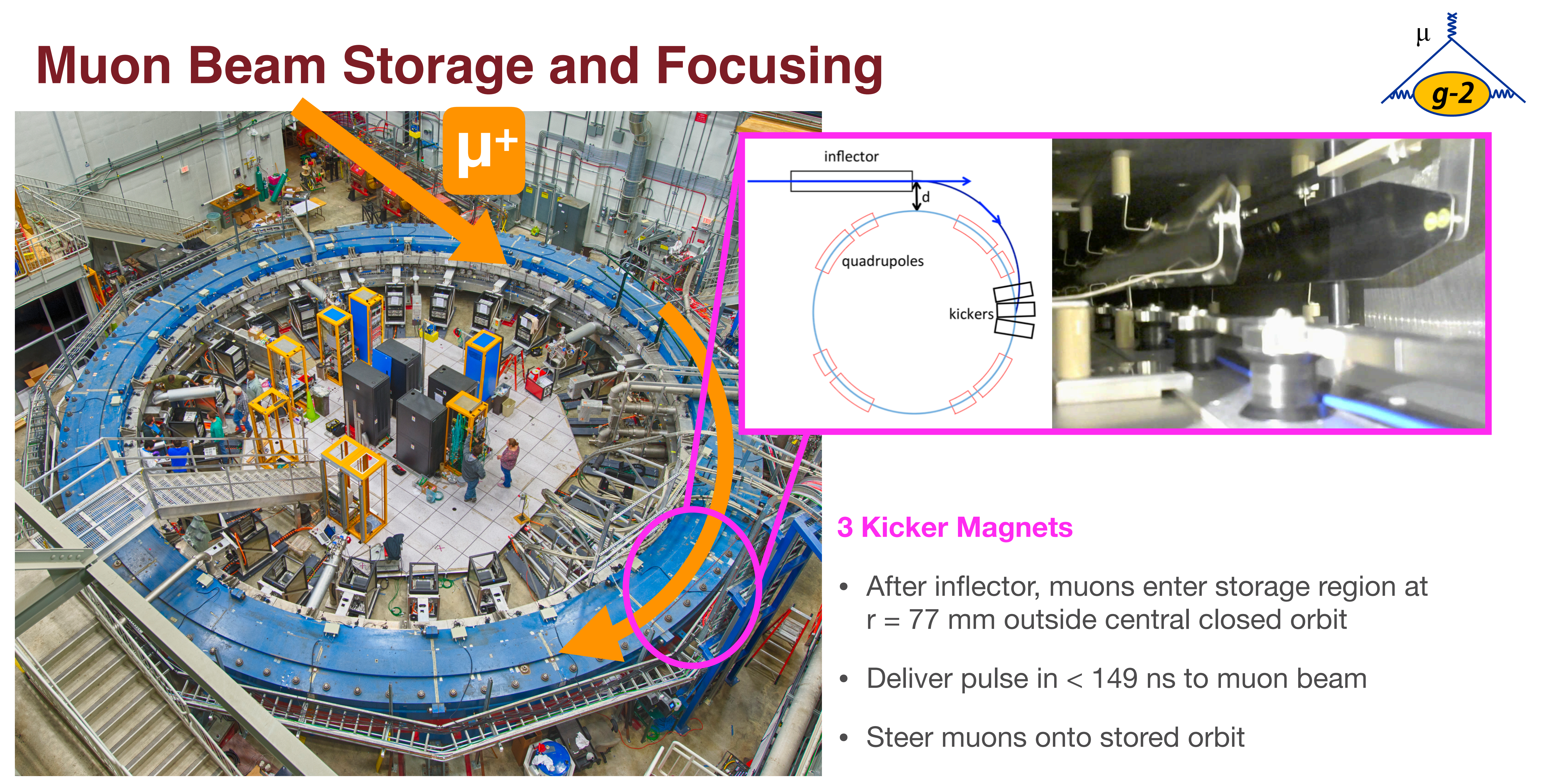


\section{Muon Beam Storage and Focusing}
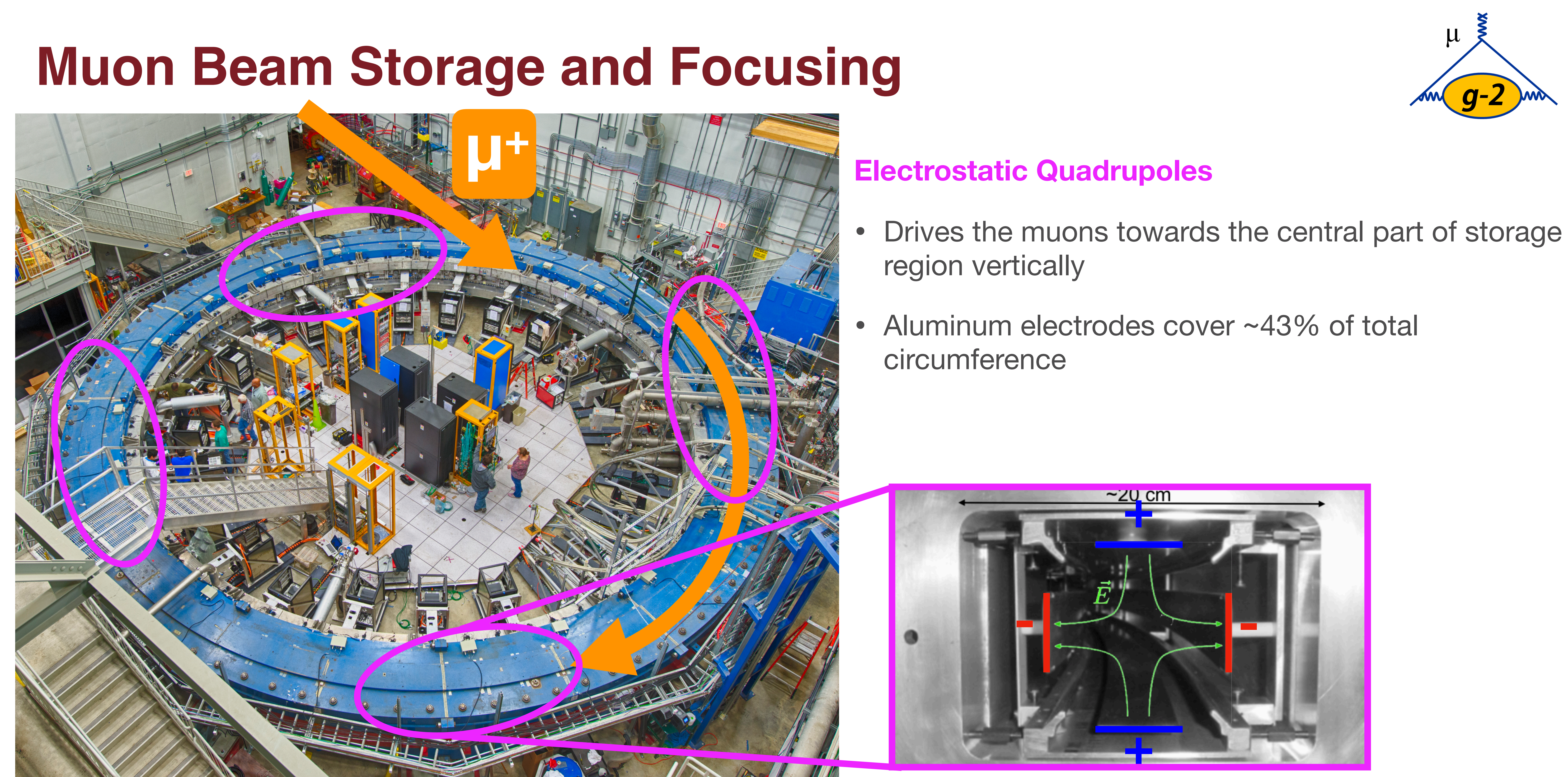


\section{Measuring Muon Spin Precession $\left(\omega_{a}\right)$}
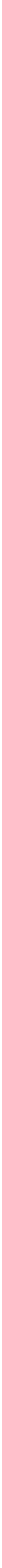

24 finely-segmented $\mathrm{PbF}_{2}$ crystal calorimeters

- Self-analyzing decay: $\mu^{+} \rightarrow e^{+} \bar{\nu}_{\mu} \nu_{e}$

- Highest-energy e+ emitted preferentially along muon spin

- Results in sinusoidally-oscillating arrival time of these $\mathrm{e}^{+}$in calorimeters 


\section{Measuring Muon Spin Precession $\left(\omega_{a}\right)$}

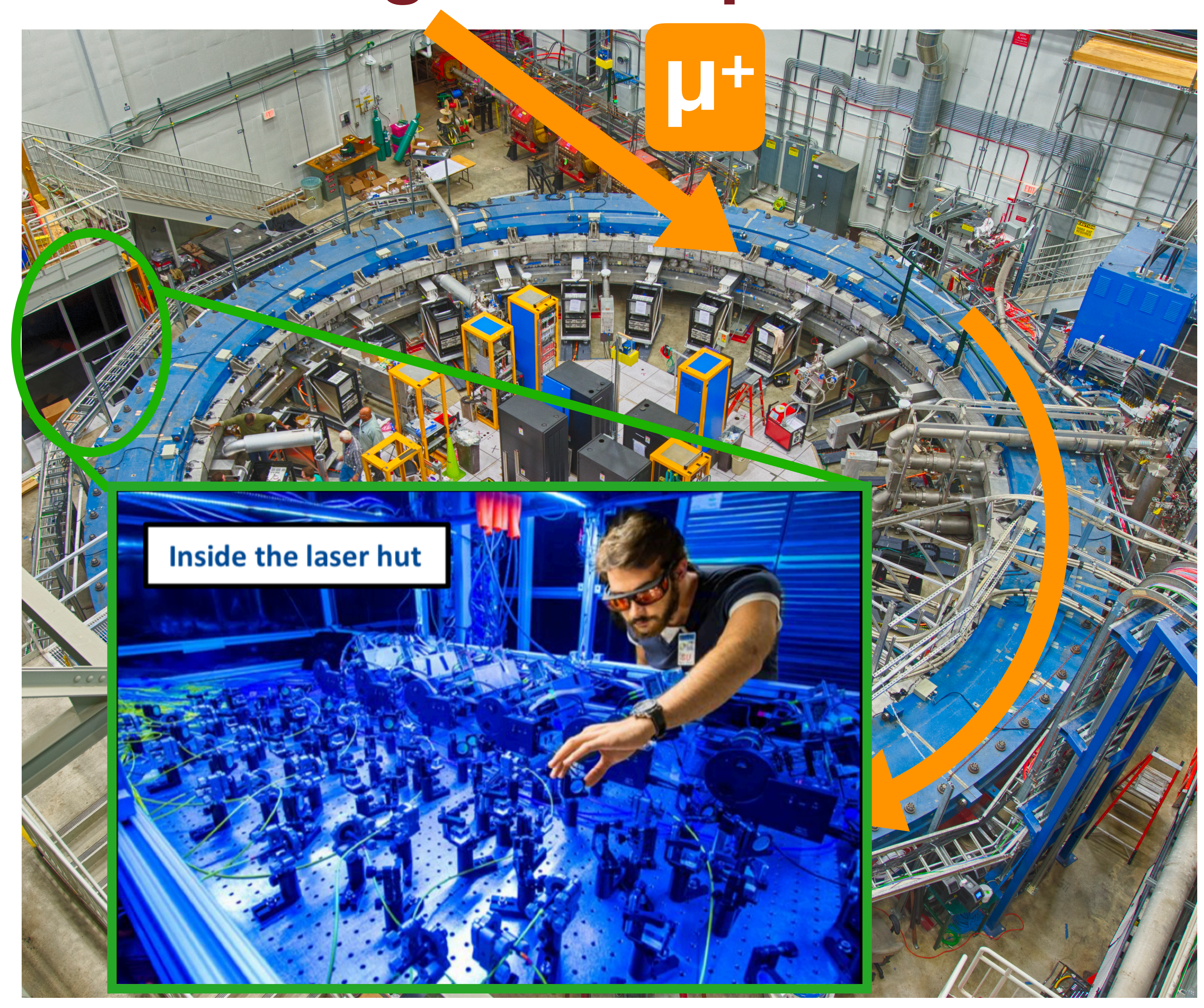

Laser System

- Calibrate calorimeter gain response throughout data taking

- Demonstrated stability to $10^{-4} / \mathrm{hr}$

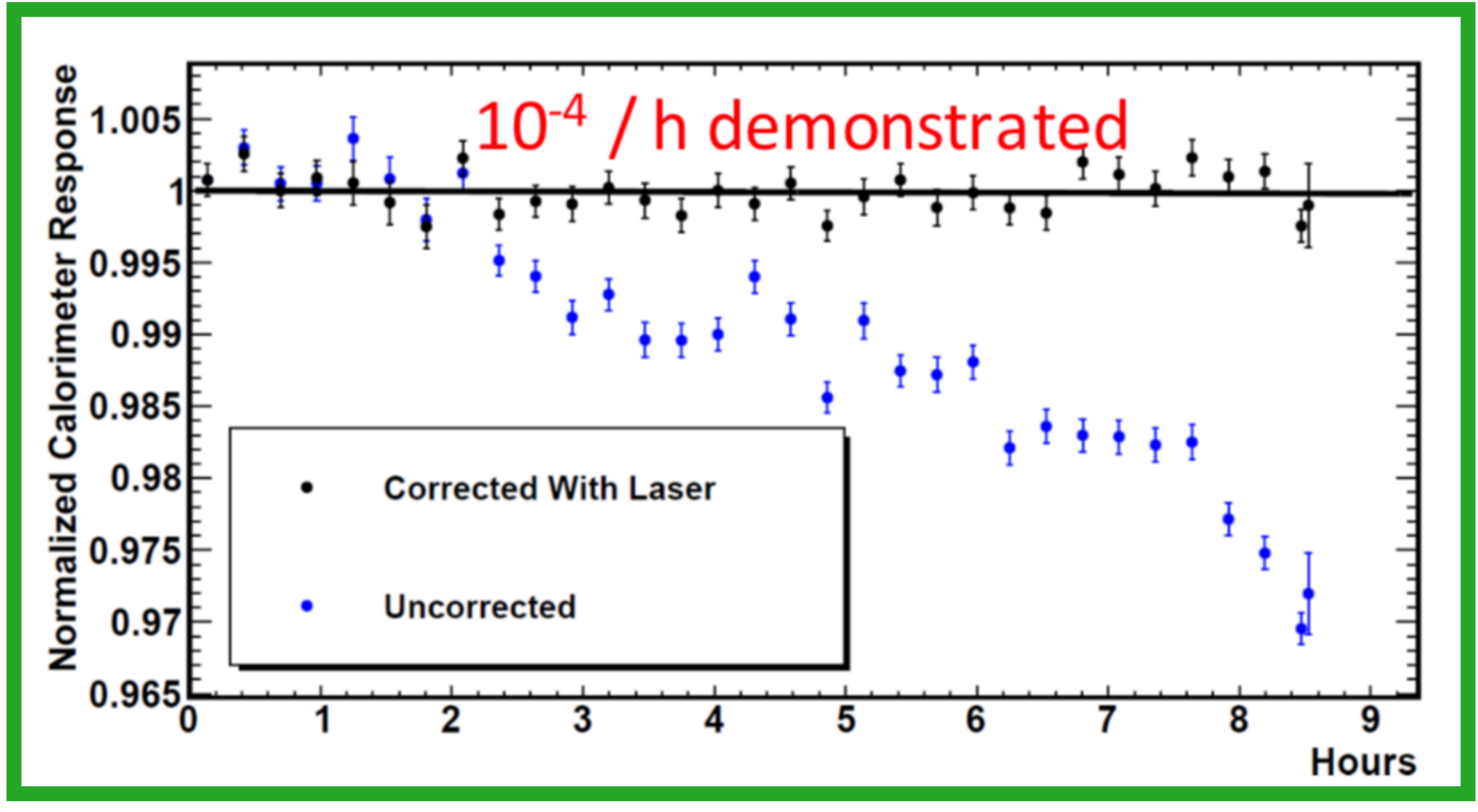

29 David Flay I Measuring the Muon Anomalous Magnetic Moment to High Precision 


\section{Measuring Muon Spin Precession $\left(\omega_{a}\right)$}

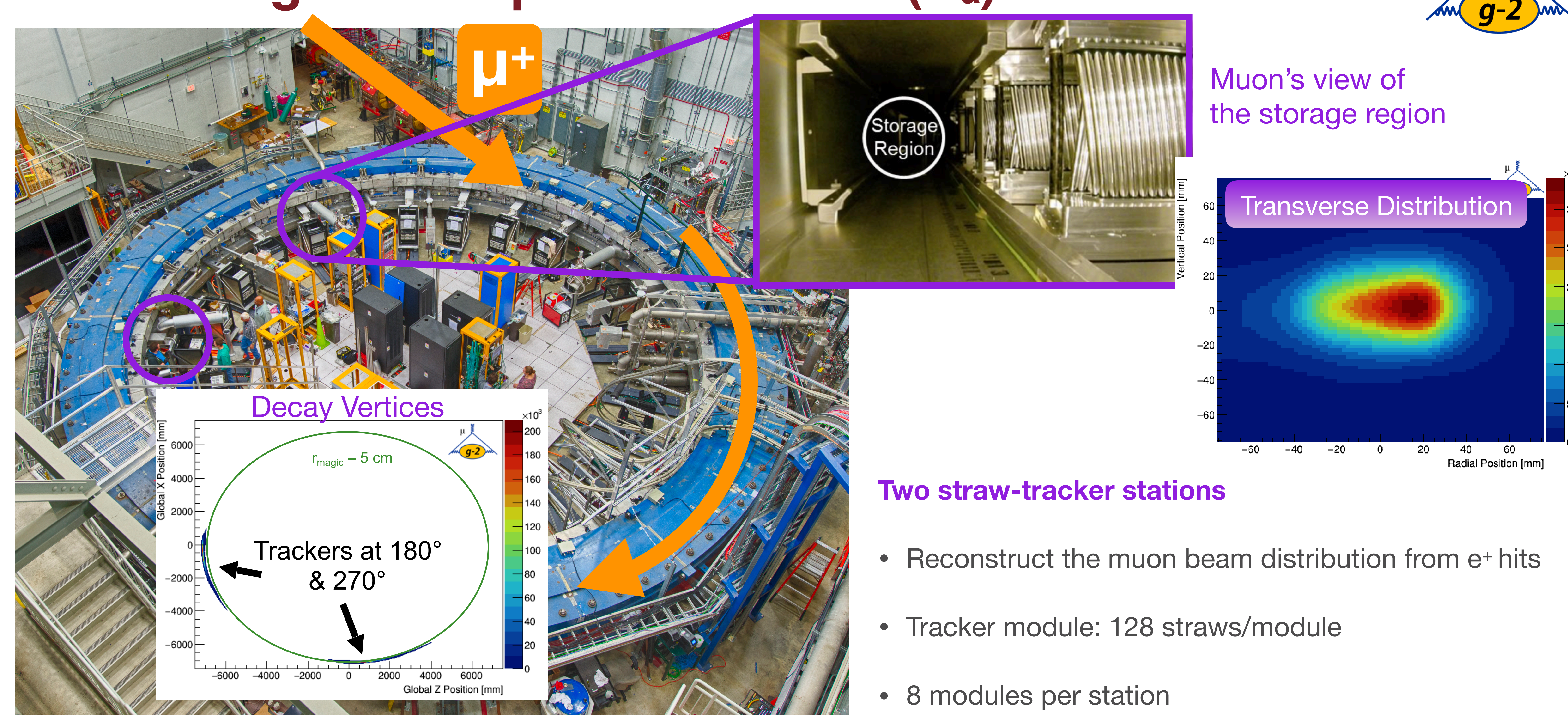

30 David Flay I Measuring the Muon Anomalous Magnetic Moment to High Precision 


\section{Monitoring and Mapping the Magnetic Field}
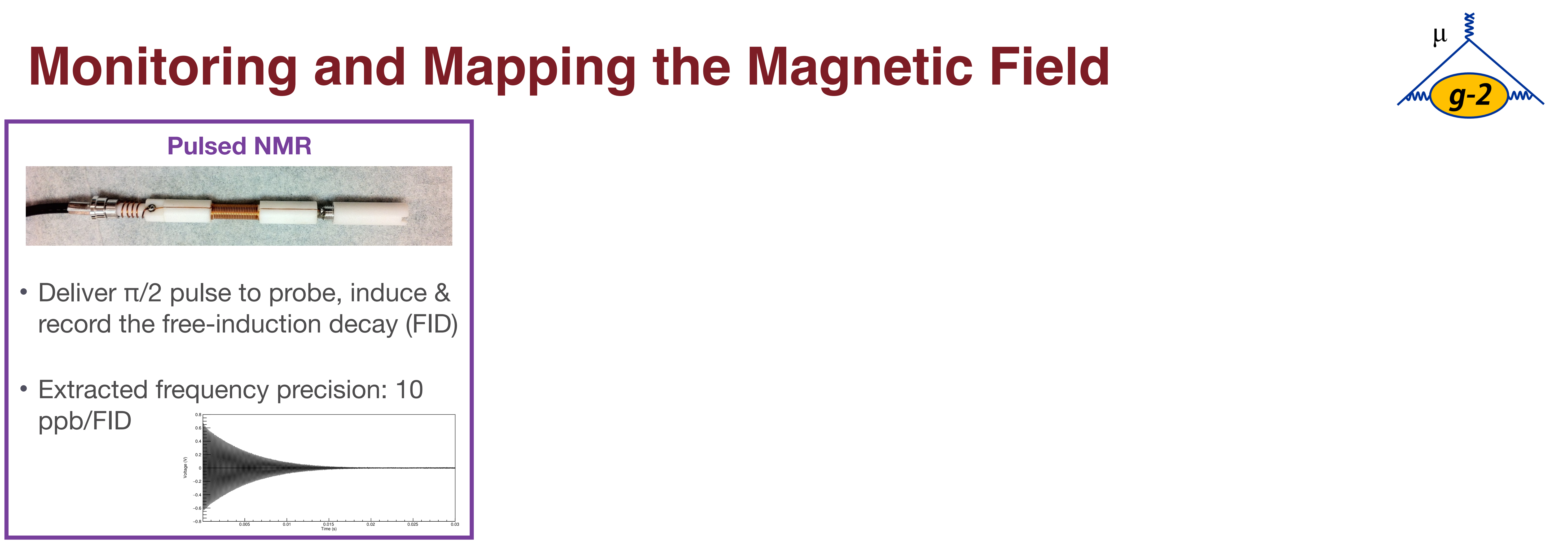


\section{Monitoring and Mapping the Magnetic Field}

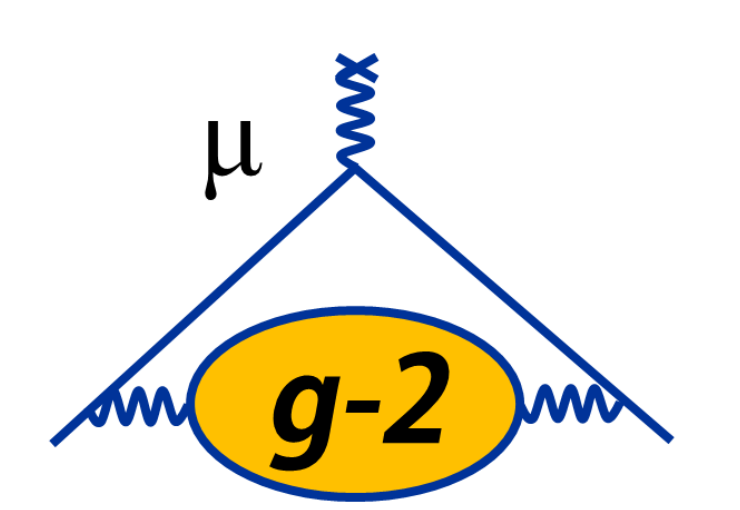

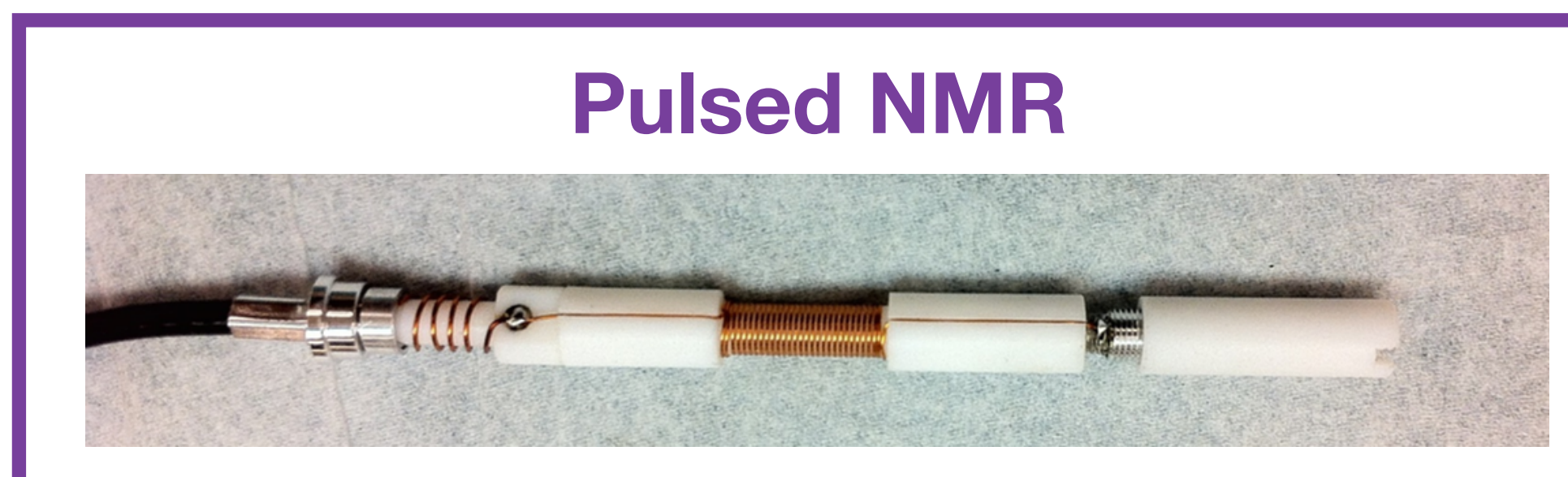

- Deliver $\pi / 2$ pulse to probe, induce \& record the free-induction decay (FID)

- Extracted frequency precision: 10 ppb/FID

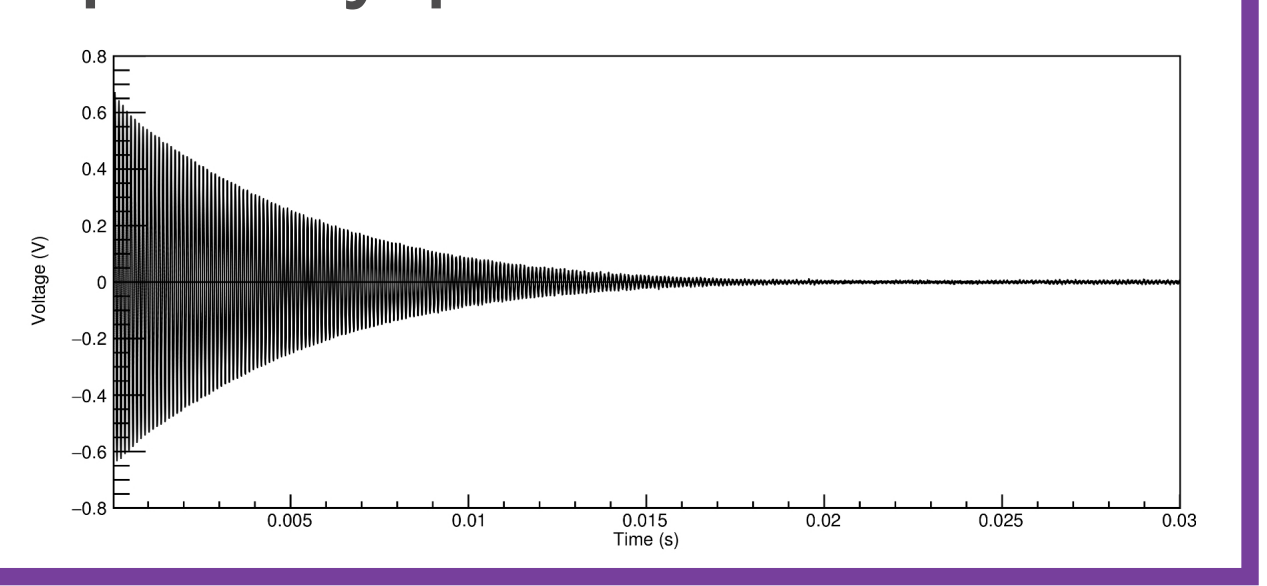

Fixed probes on vacuum chambers

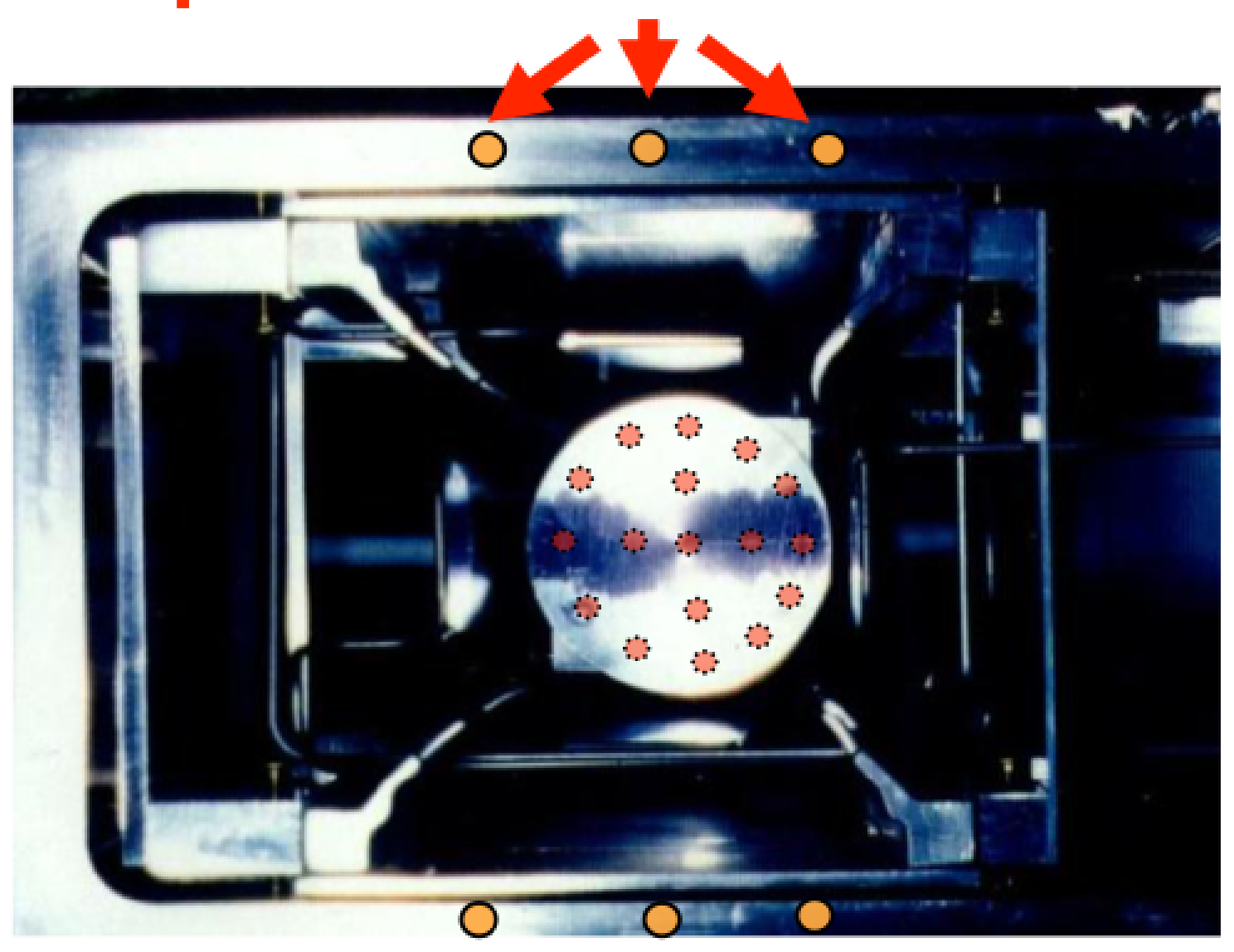

- Measure field while muons are in ring - 378 probes outside storage region
Trolley matrix of 17 NMR probes

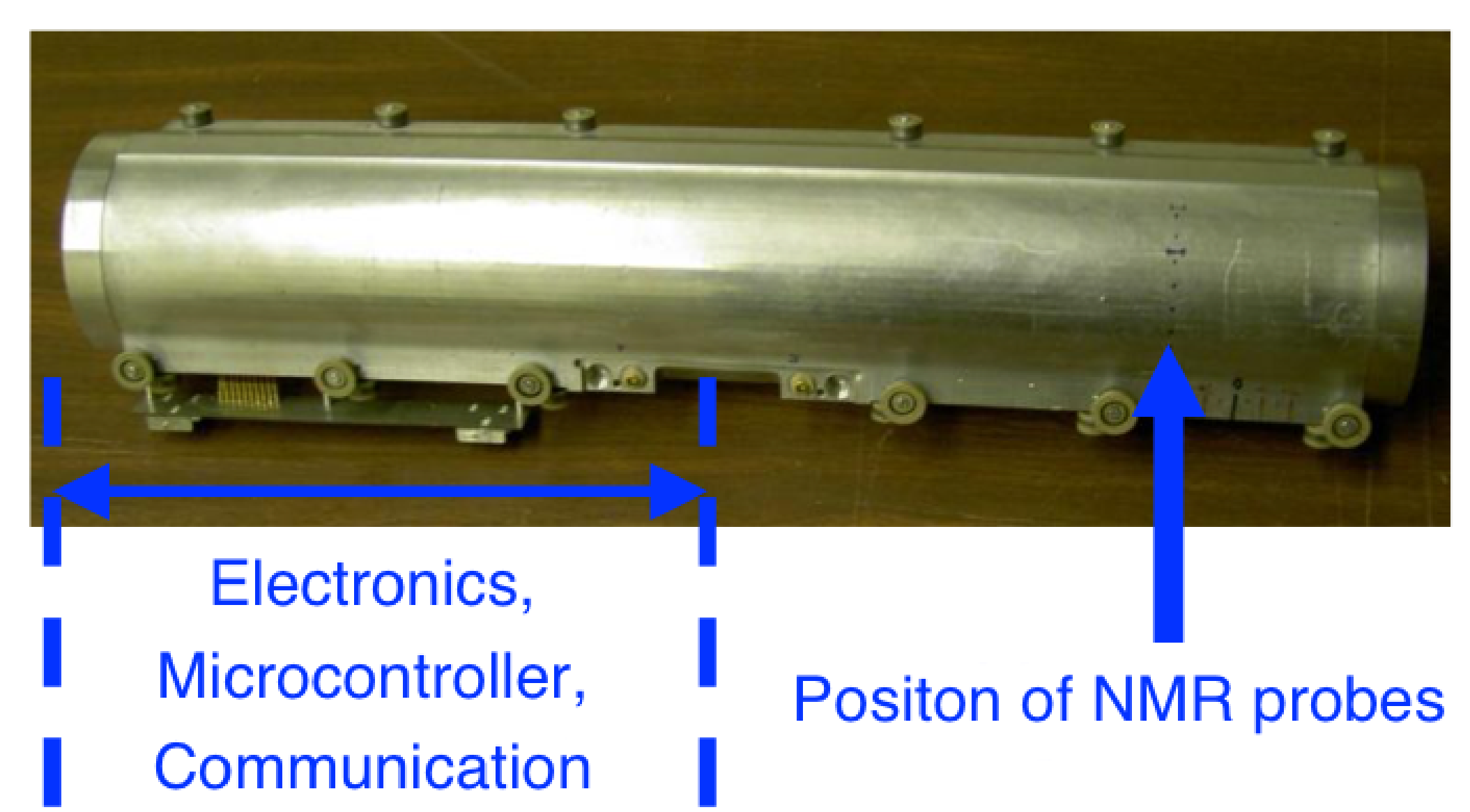

- Measure field in storage region during specialized runs when muons are not being stored 


\section{Monitoring and Mapping the Magnetic Field}
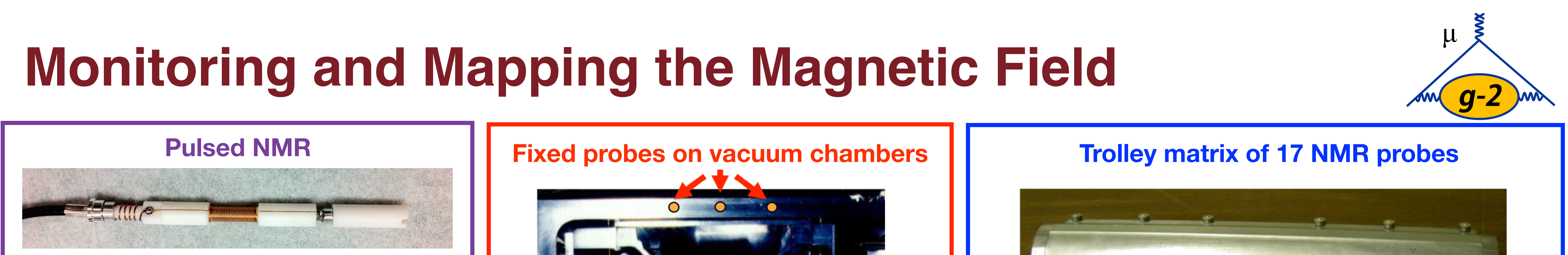

- Deliver $\pi / 2$ pulse to probe, induce \& record the free-induction decay (FID)

- Extracted frequency precision: 10 $\mathrm{ppb} / \mathrm{FID}$

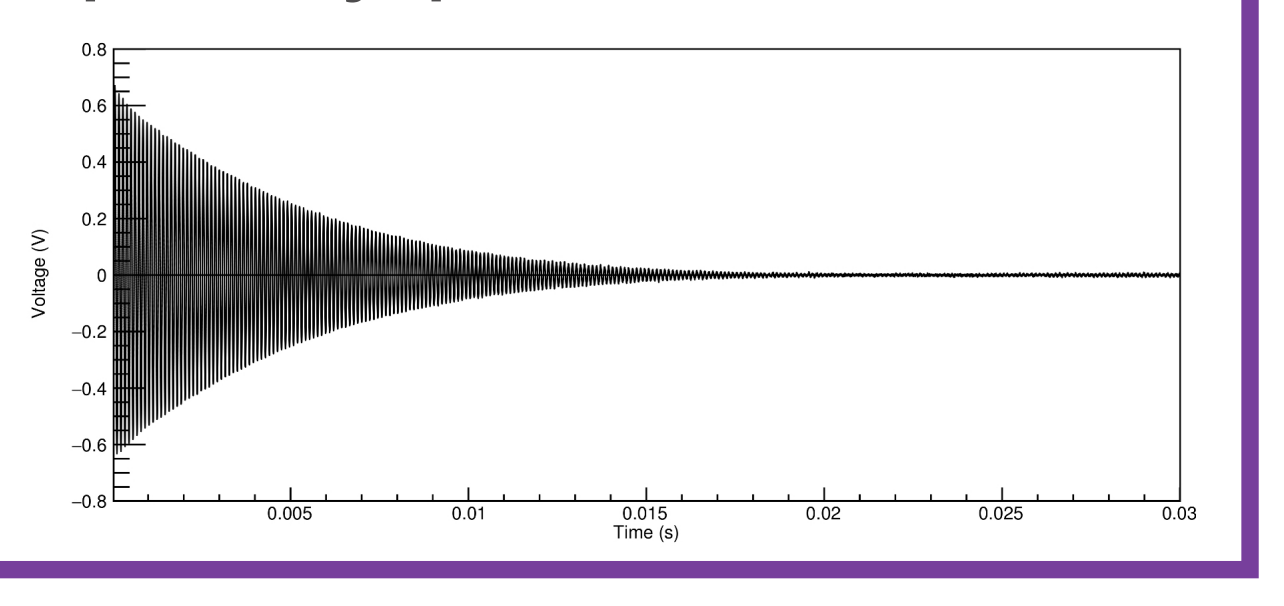

Fixed probes on vacuum chambers

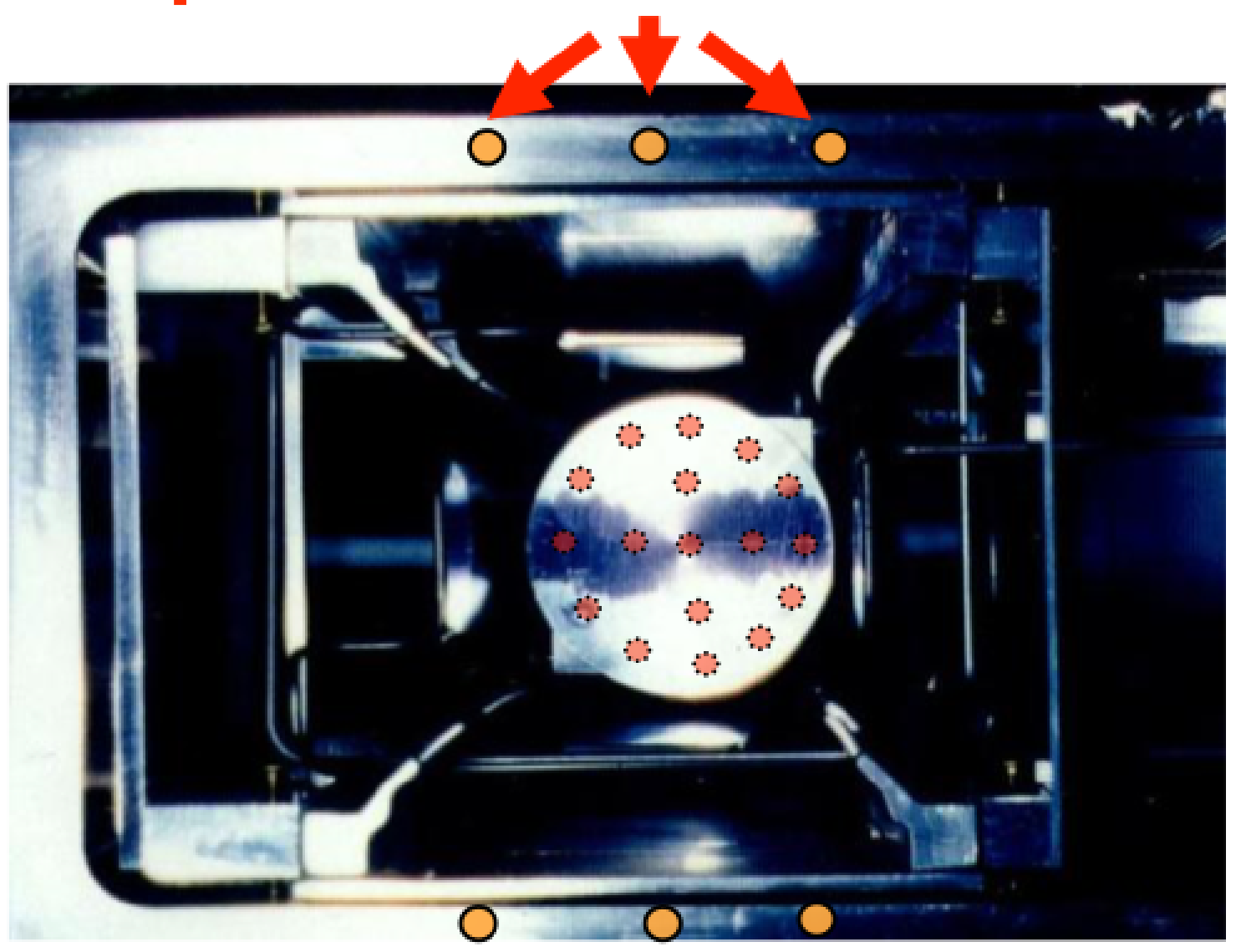

- Measure field while muons are in ring - 378 probes outside storage region

\section{Trolley matrix of 17 NMR probes}

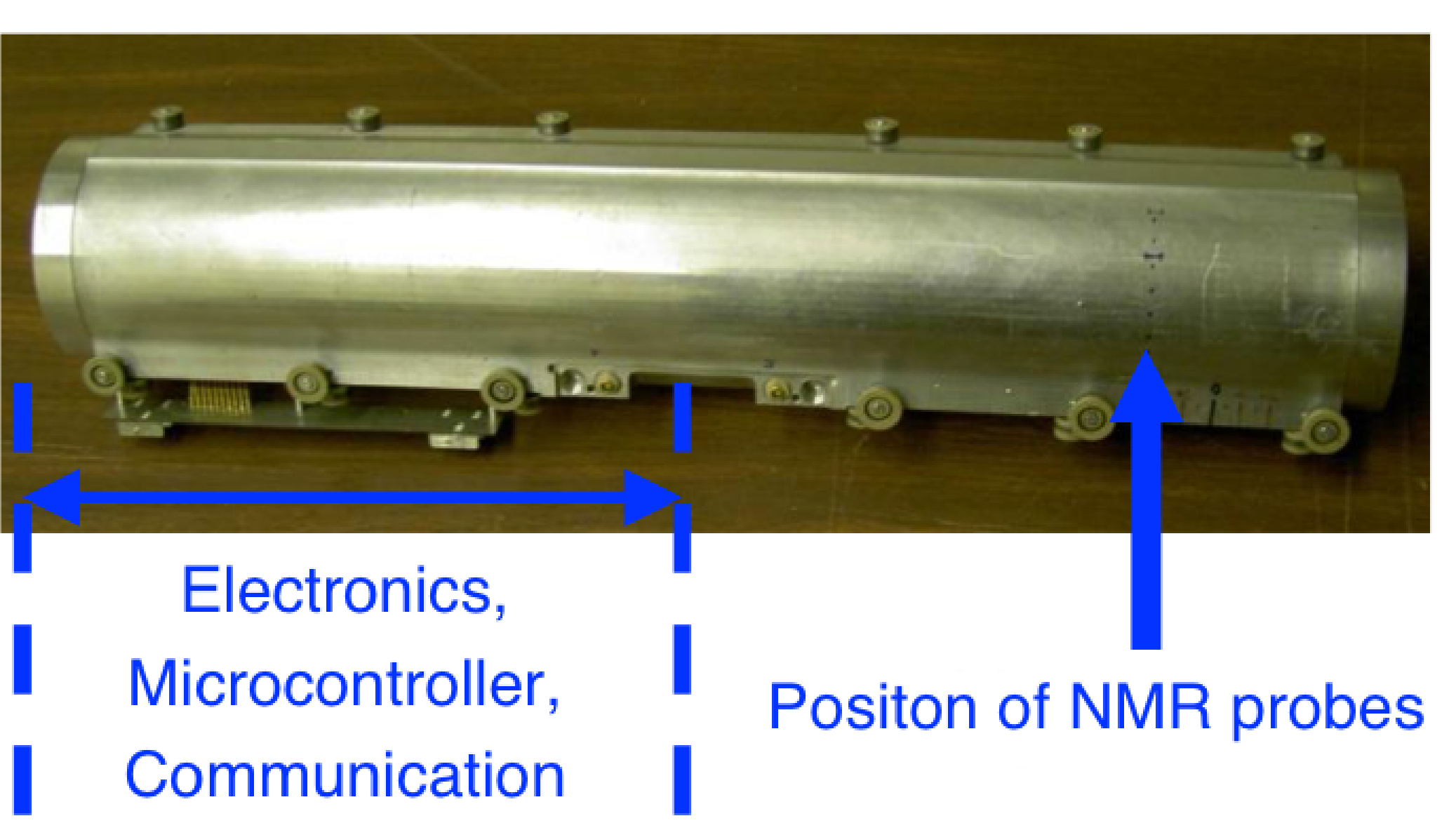

- Measure field in storage region during specialized runs when muons are not being stored

- Trolley probes calibrated to free-proton Larmor frequency

- Calibrate trolley probes using a special probe that uses a water sample

- Measurements in specially-shimmed region of ring
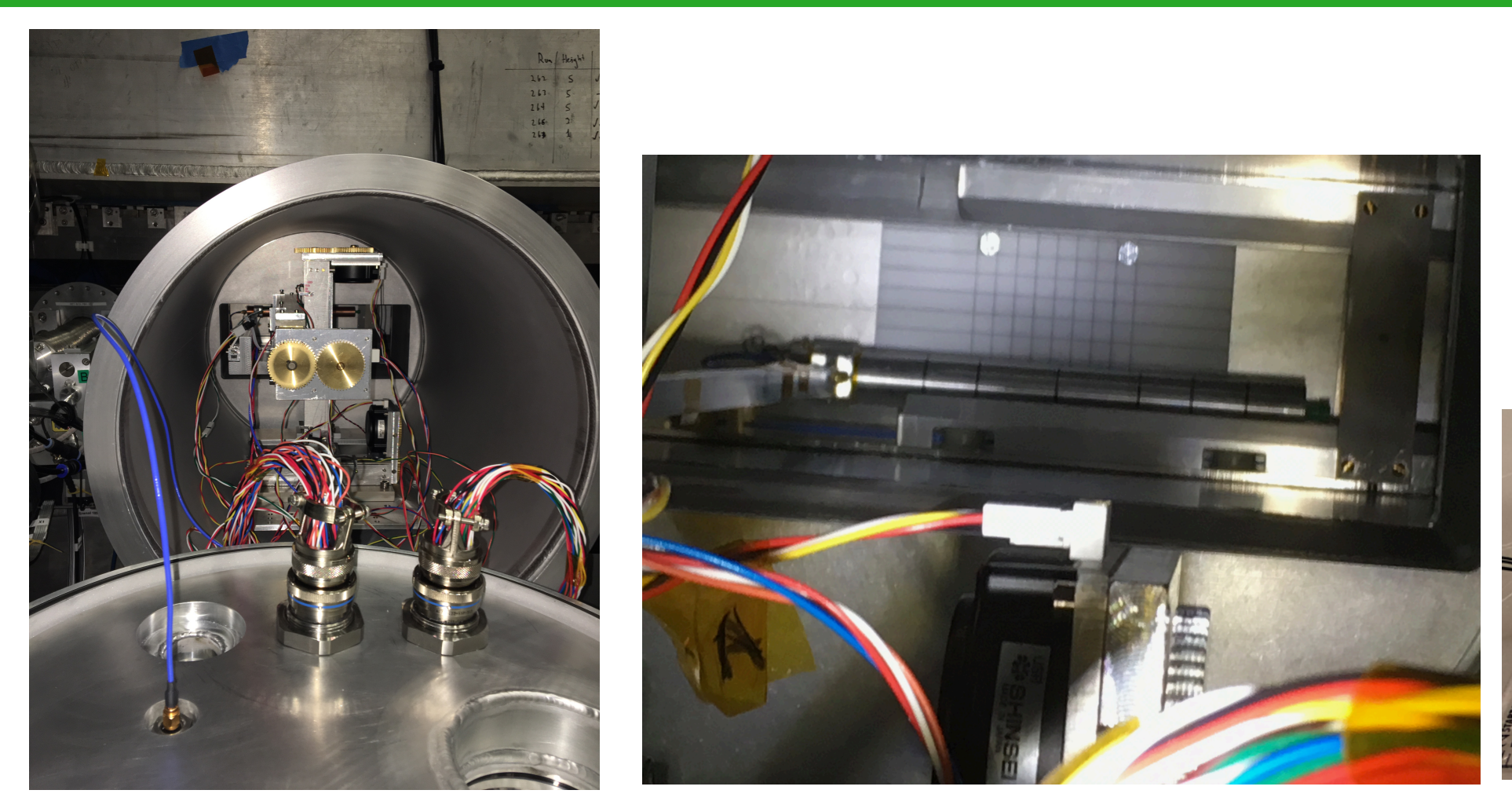

Plunging Probe

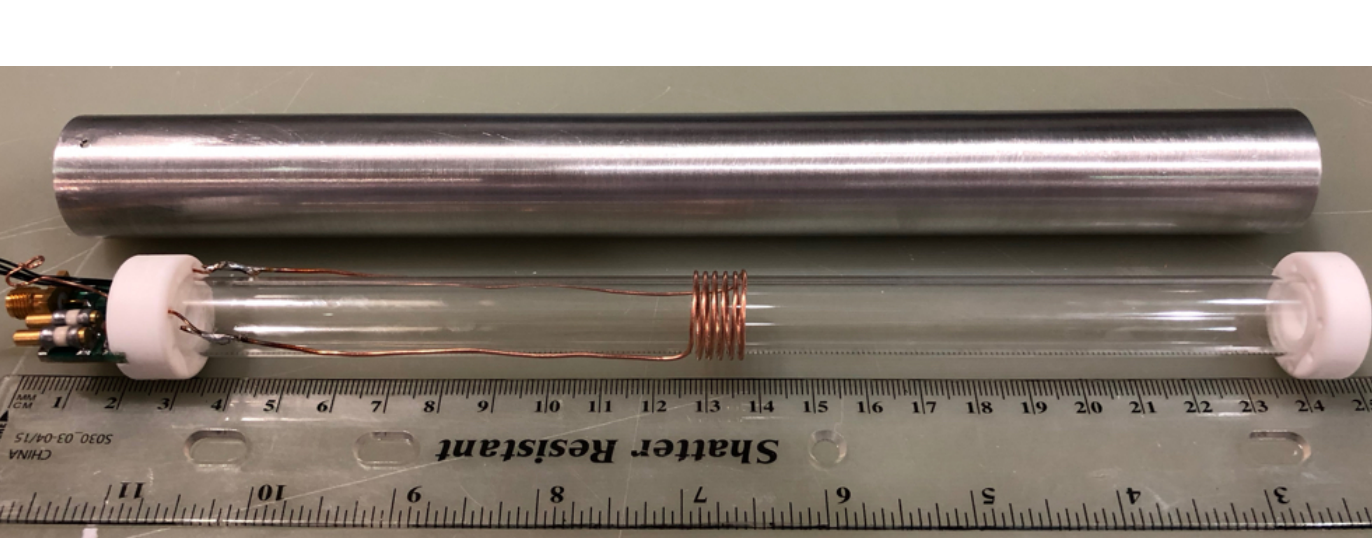

34 David Flay I Measuring the Muon Anomalous Magnetic Moment to High Precision 


\section{Systematic Uncertainty Comparison: E821 and E989}

$$
a_{\mu}=\frac{\omega_{a}}{\tilde{\omega}_{p}} \frac{\mu_{p}}{\mu_{e}} \frac{m_{\mu}}{m_{e}} \frac{g_{e}}{2}
$$

\begin{tabular}{|c|c|c|}
\hline \multicolumn{3}{|c|}{$\omega_{a}$ Goal: Factor of 3 Improvement } \\
\hline Category & E821 (ppb) & E989 Goal (ppb) \\
Gain Changes & 120 & 20 \\
Lost Muons & 90 & 20 \\
Pileup & 80 & 40 \\
Horizontal CBO & 70 & $<30$ \\
E-field/pitch & 110 & 30 \\
\hline Quadrature Sum & $\mathbf{2 1 4}$ & $\mathbf{7 0}$ \\
\hline
\end{tabular}

- New hardware (calorimeters, trackers, NMR)

- Improved analysis techniques

- Reduce uncertainties by at least a factor of 2.5

\begin{tabular}{|c|c|c|}
\hline \multicolumn{3}{|c|}{$\omega_{\mathrm{p}}$ Goal: Factor of 2.5 Improvement } \\
Category & $\mathbf{E 8 2 1}$ (ppb) & E989 Goal (ppb) \\
\hline Field Calibration & 50 & 35 \\
Trolley Measurements & 50 & 30 \\
Fixed Probe Interpolation & 70 & 30 \\
Muon Convolution & 30 & 10 \\
Time-Dependent Fields & - & 5 \\
\hline Others & 100 & 50 \\
\hline Quadrature Sum & $\mathbf{1 7 0}$ & $\mathbf{7 0}$ \\
\hline
\end{tabular}

UMassAmherst 


\section{Run 1 Overview}

- Data taking period: April-July 2018

- A number of changing conditions as we optimized hardware

- Accumulated $\sim 1.1 \times$ BNL statistics (after data quality cuts) $-\delta \omega_{a}($ stat $) \sim 410 \mathrm{ppb}$

- Field uniformity $\sim 2 x$ better than BNL
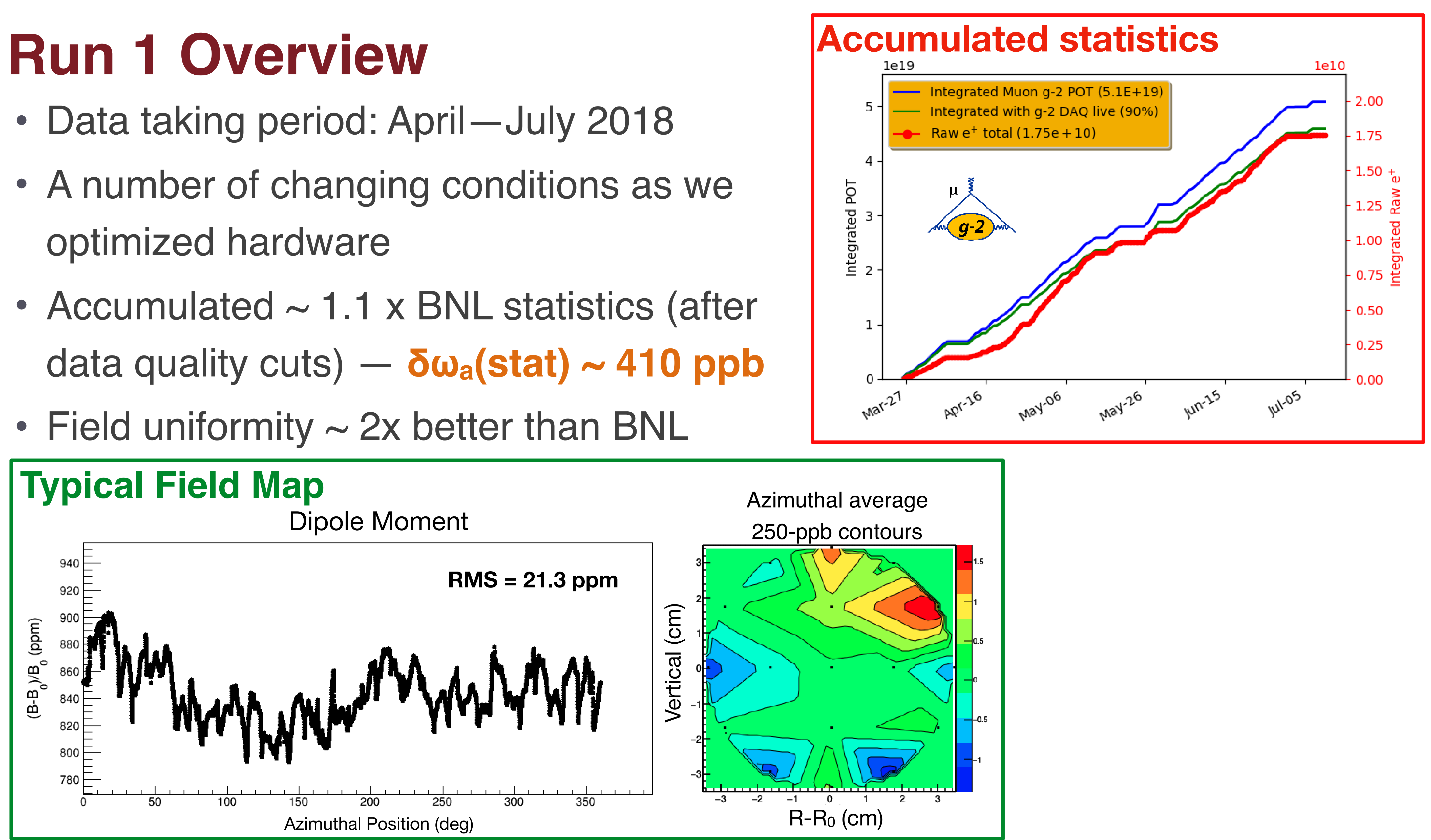

36 David Flay I Measuring the Muon Anomalous Magnetic Moment to High Precision 


\section{Run 2 Overview}

- Data taking period: March-July 2019

- Contiguous data set

- Accumulated $1.9 \times$ BNL statistics (before data quality cuts)

- Field uniformity in very good condition
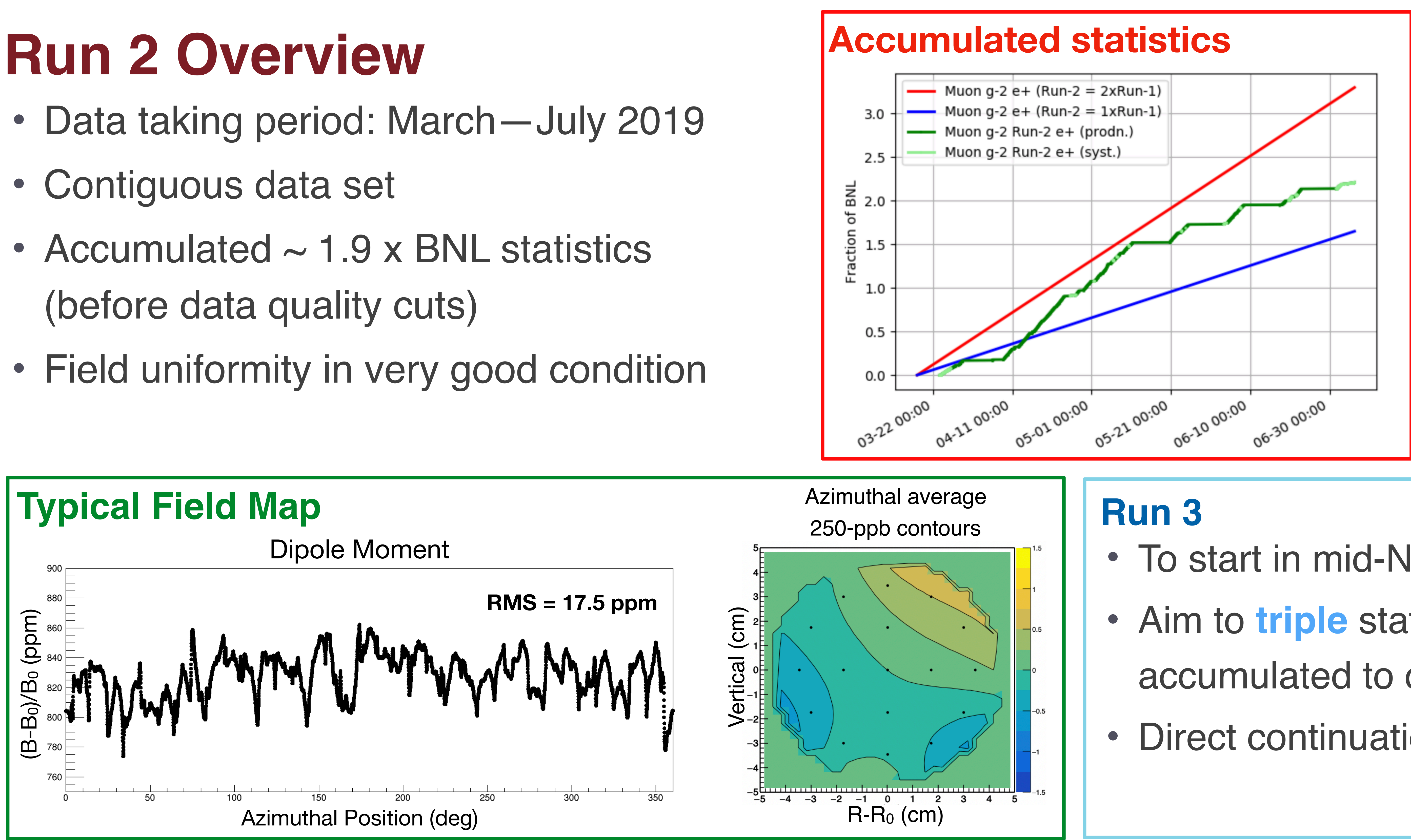

\section{Run 3}

- To start in mid-November

- Aim to triple statistics accumulated to date

- Direct continuation of Run 2 


\section{Run 1 Analysis Status - $\omega_{a}$}

$$
a_{\mu}=\frac{\omega_{a}}{\tilde{\omega}_{p}} \frac{\mu_{p}}{\mu_{e}} \frac{m_{\mu}}{m_{e}} \frac{g_{e}}{2}
$$




\section{Run 1 Analysis Status: $\omega_{a}$}

- Account for a number of effects that can affect the extraction of $\omega_{a}$

$$
N(t)=N_{0} e^{-t / \tau}\left[1-A \cos \left(\omega_{a} t+\phi\right)\right]
$$




\section{Run 1 Analysis Status: $\omega_{a}$}

- Account for a number of effects that can affect the extraction of $\omega_{a}$

$$
N(t)=N_{0} e^{-t / \tau}\left[1-A \cos \left(\omega_{a} t+\phi\right)\right]
$$

\section{Detector effects}

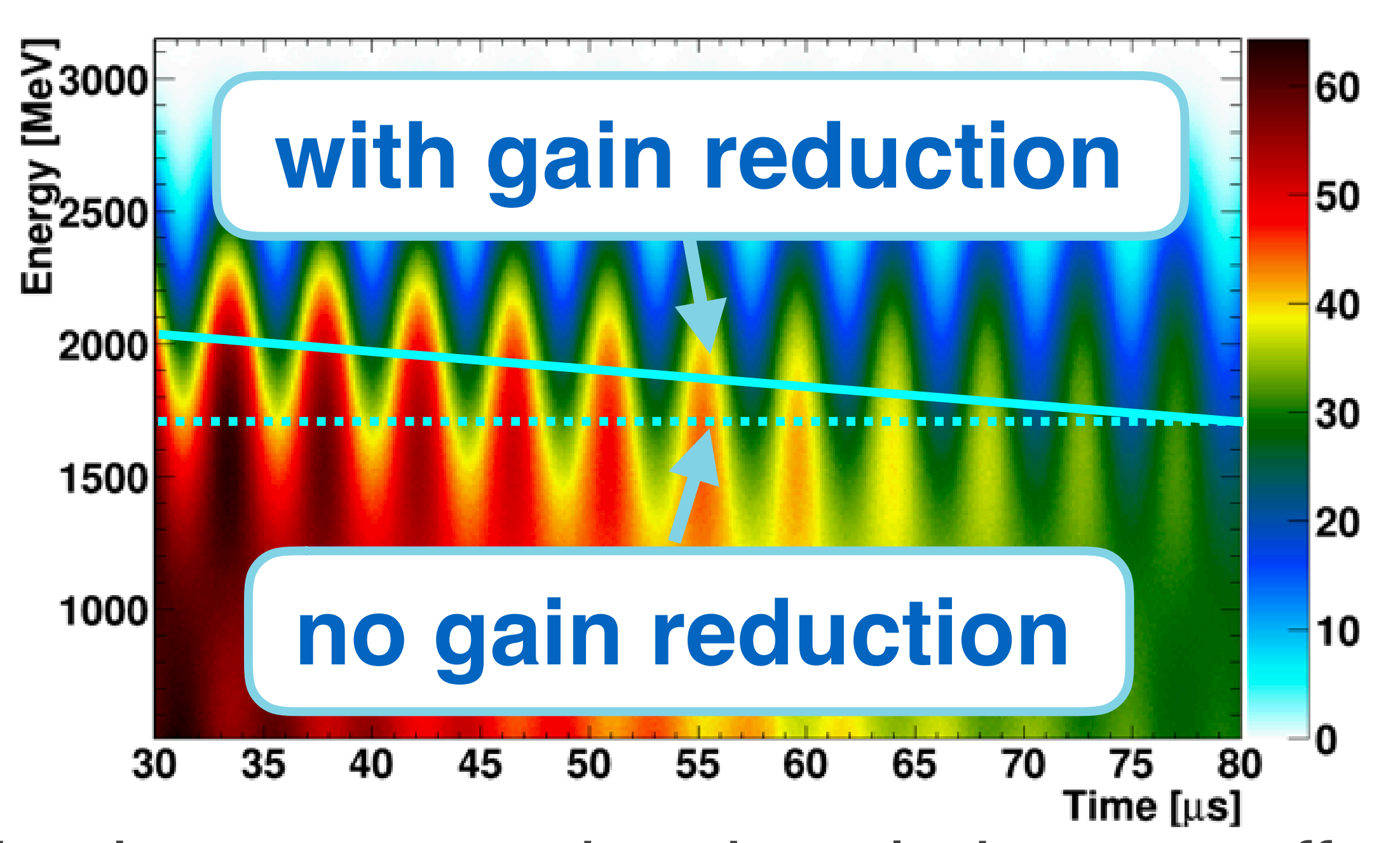

- Gain changes over time in calorimeters affects phase of signal: $N \rightarrow N(t), A \rightarrow A(t), \phi \rightarrow \phi(t)$

- Laser system provides corrections 


\section{Run 1 Analysis Status: $\omega_{a}$}

- Account for a number of effects that can affect the extraction of $\omega_{a}$

$$
N(t)=N_{0} e^{-t / \tau}\left[1-A \cos \left(\omega_{a} t+\phi\right)\right]
$$

\section{Detector effects}

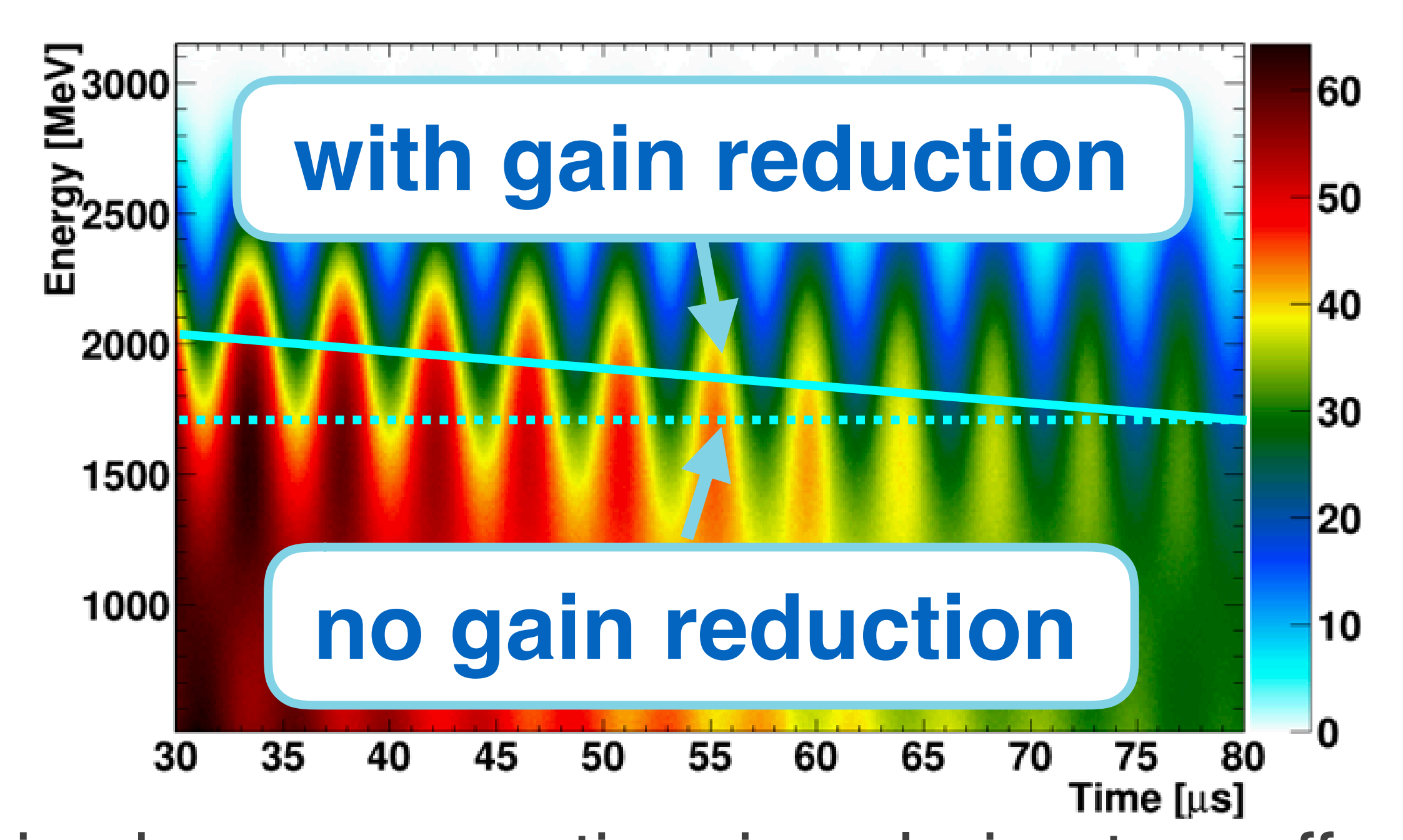

- Gain changes over time in calorimeters affects phase of signal: $N \rightarrow N(t), A \rightarrow A(t), \phi \rightarrow \phi(t)$

- Laser system provides corrections

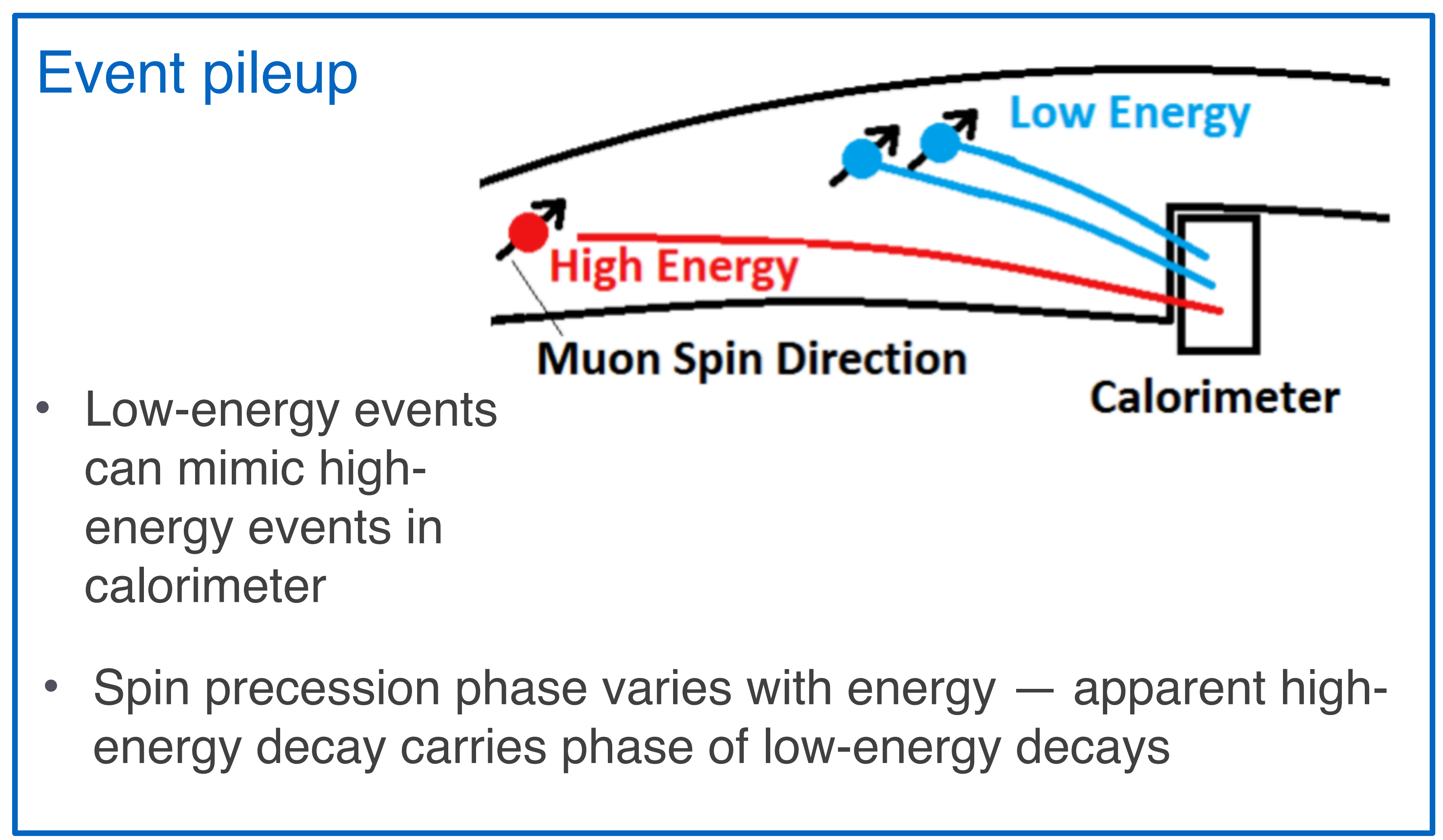




\section{Run 1 Analysis Status: $\omega_{a}$}

- Account for a number of effects that can affect the extraction of $\omega_{a}$

$$
N(t)=N_{0} e^{-t / \tau}\left[1-A \cos \left(\omega_{a} t+\phi\right)\right]
$$

\section{Beam dynamics}

Muon losses

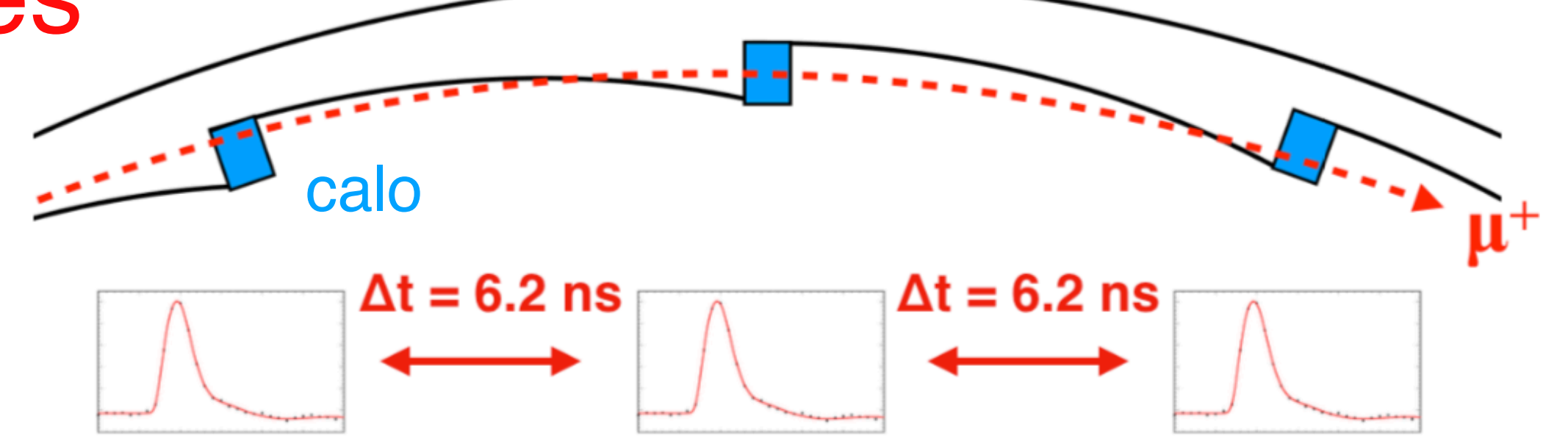

- Muons can leave storage ring by decaying or escaping

- Exhibit specific signature in multiple calorimeters

- Amplitude $N_{0}$ scaled by:

$$
\Lambda(t)=1-K_{\operatorname{loss}} \int_{0}^{t} e^{t^{\prime} / \tau} L\left(t^{\prime}\right) d t^{\prime}
$$




\section{Run 1 Analysis Status: $\omega_{a}$}

- Account for a number of effects that can affect the extraction of $\omega_{\mathrm{a}}$

$$
N(t)=N_{0} e^{-t / \tau}\left[1-A \cos \left(\omega_{a} t+\phi\right)\right]
$$

\section{Beam dynamics}

Muon losses

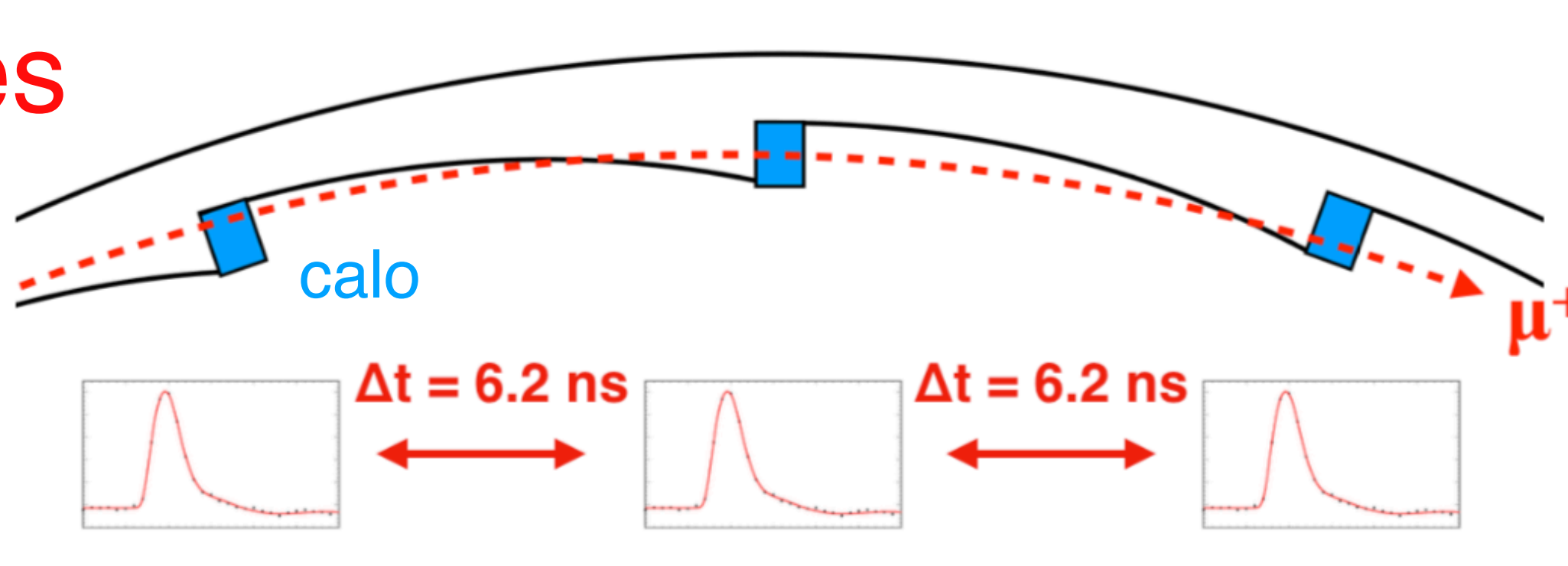

- Muons can leave storage ring by decaying or escaping

- Exhibit specific signature in multiple calorimeters

- Amplitude $\mathrm{N}_{0}$ scaled by:

$$
\Lambda(t)=1-K_{\operatorname{loss}} \int_{0}^{t} e^{t^{\prime} / \tau} L\left(t^{\prime}\right) d t^{\prime}
$$

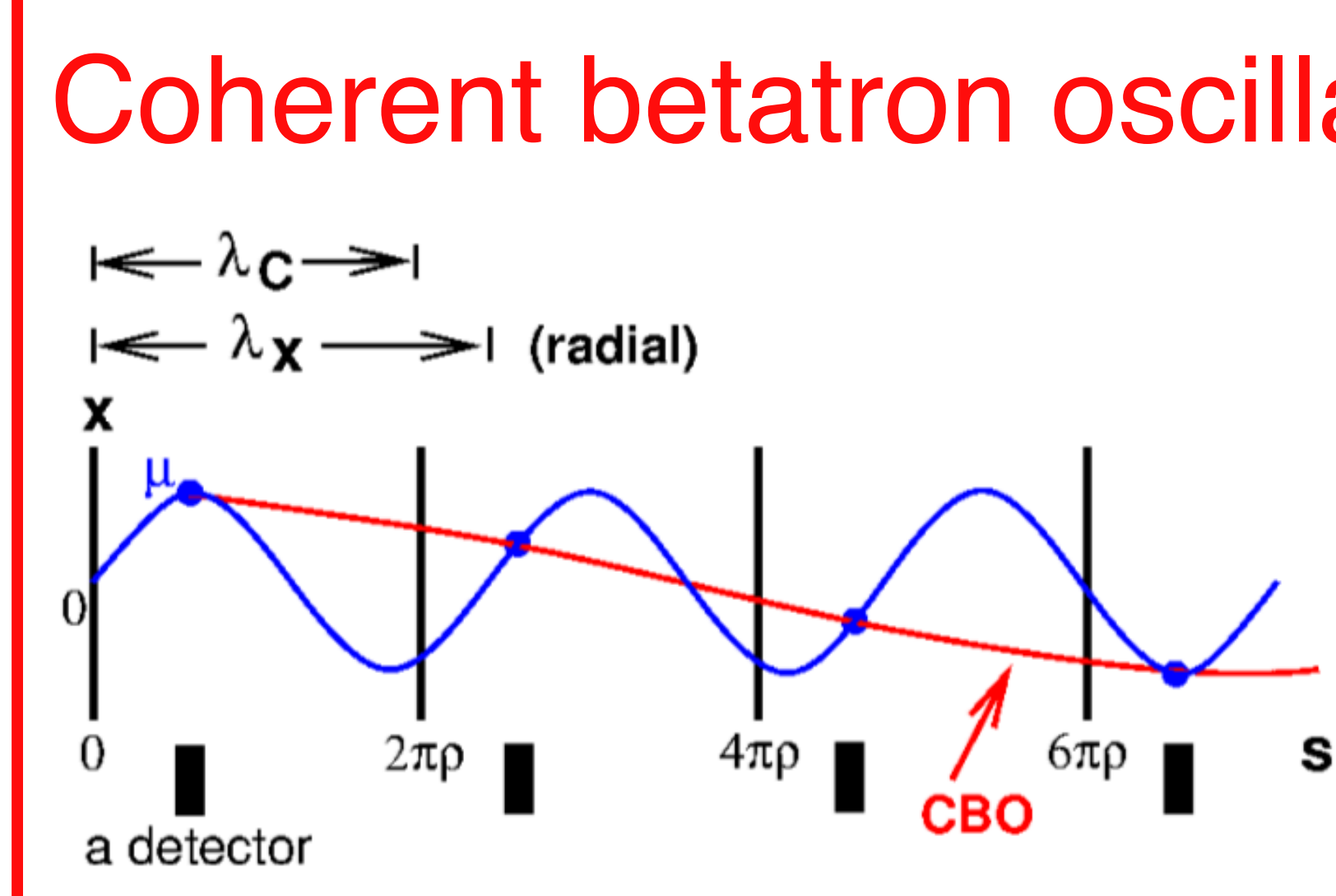

- Acceptance of calorimeters affected by coherent radial beam motion

- Amplitude $\mathrm{N}_{0}$ scaled by:

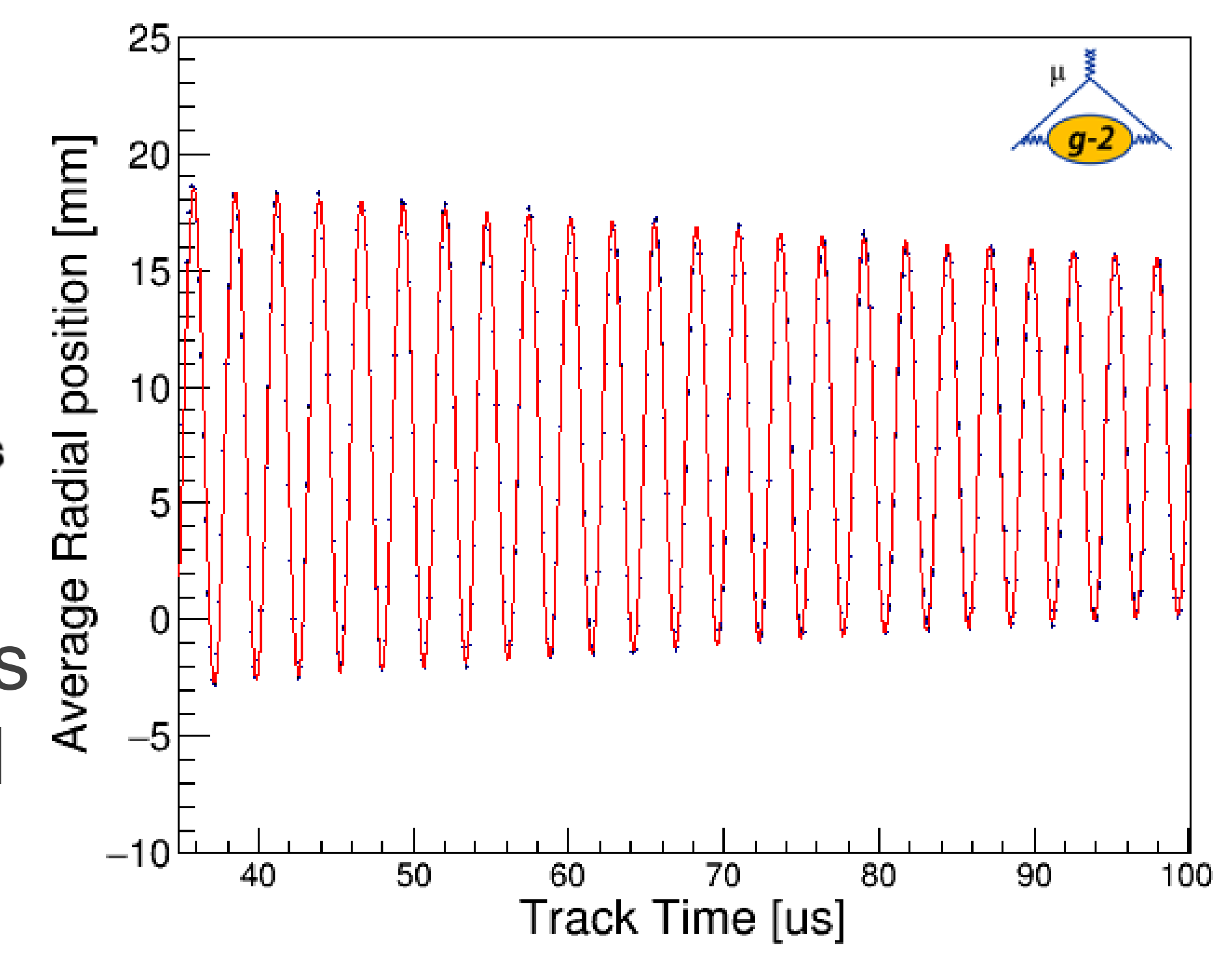

$$
C(t)=1-e^{-t / \tau_{\mathrm{CBO}}} A_{1} \cos \left(\omega_{\mathrm{CBO}} t+\phi_{1}\right)
$$




\section{Run 1 Analysis Status: $\omega_{a}$}

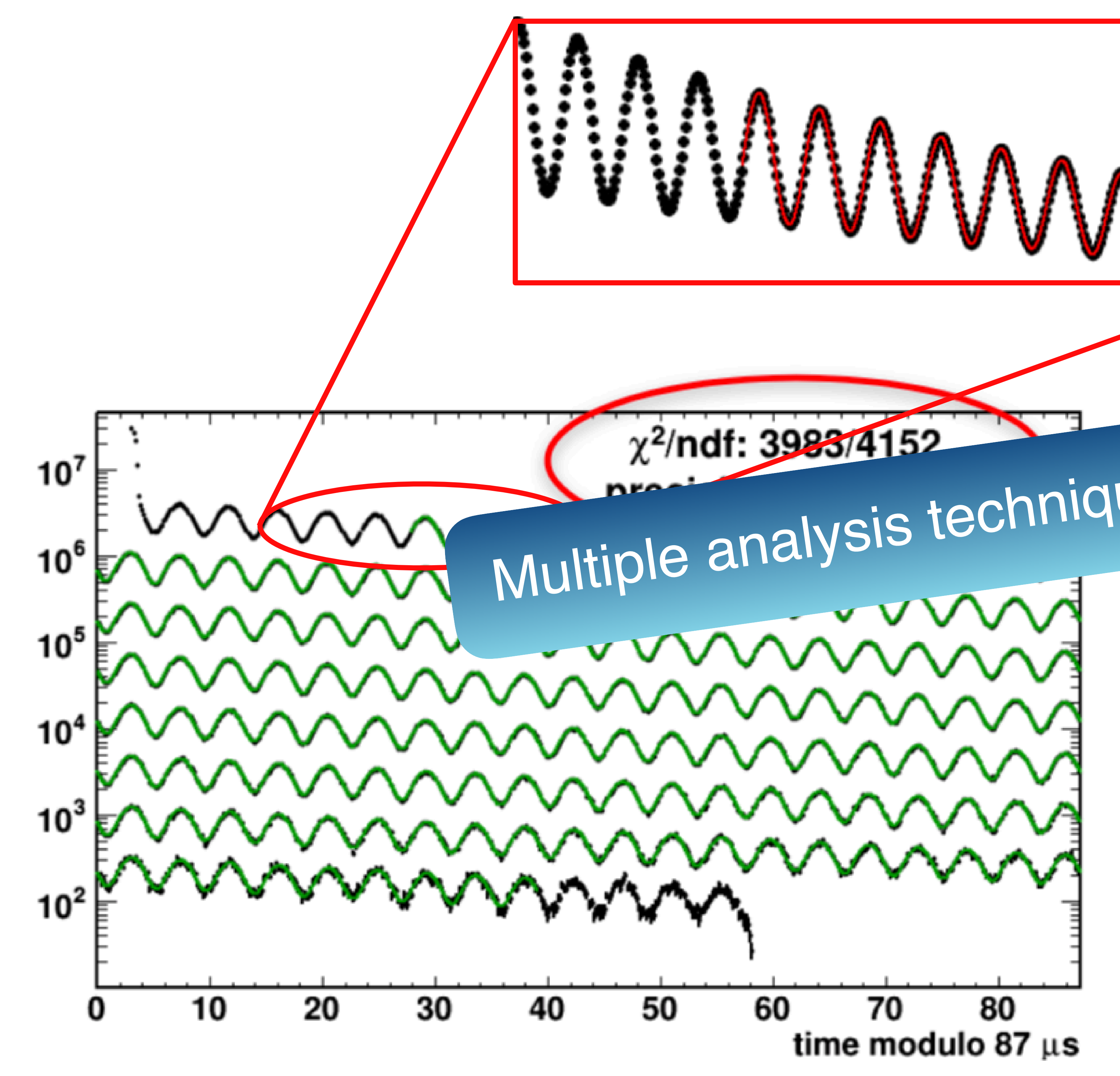

Simple five-parameter fit

FFT of fit residuals

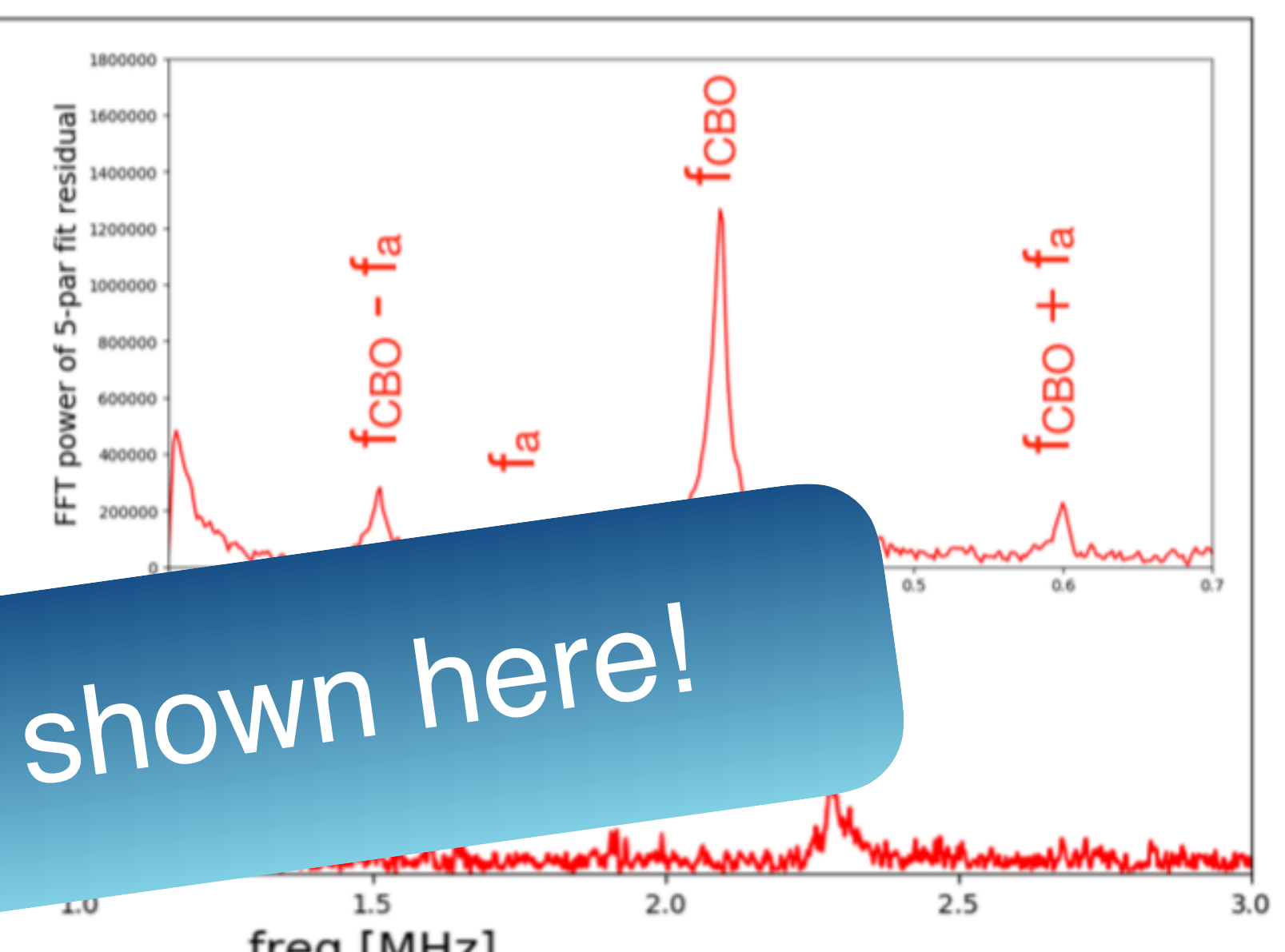

Big improvements when accounting for $\mathrm{CBO}$, lost muons,...

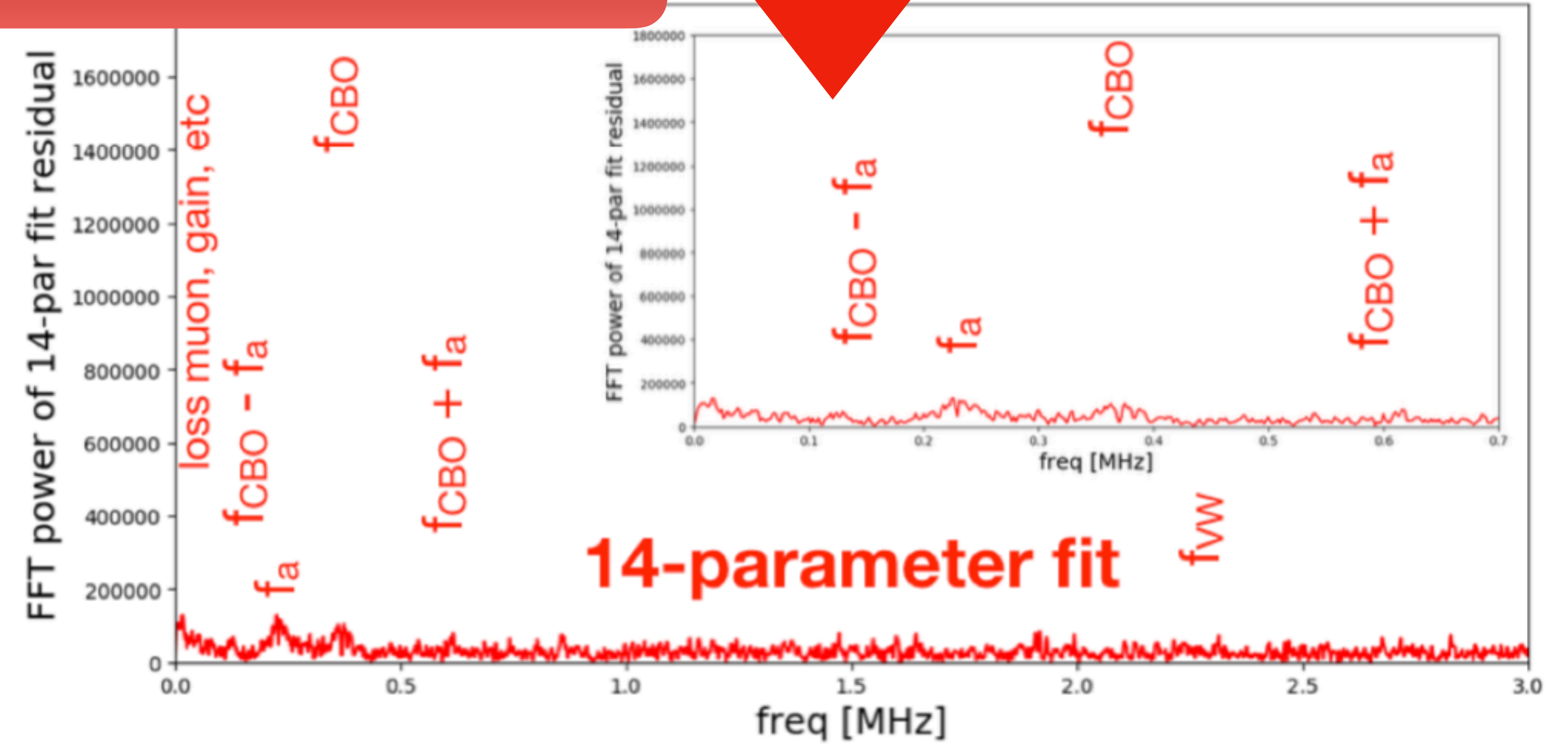

UMassAmherst 


\section{Run 1 Analysis Status: Relative Unblinding for $\omega_{a}$}

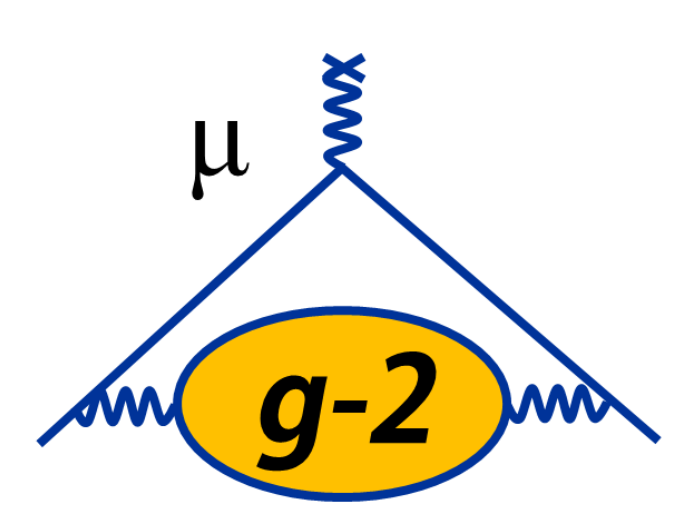

- Doubly-blinded in $\omega_{\mathrm{a}}$ measurement: Clock tuned to $40 \mathrm{MHz} \pm 25 \mathrm{ppm}$

- Analyzers' results come with random frequency offset $\omega_{a} \rightarrow \omega_{a} \pm 25$ ppm

- Recently compared results on subset of data at a common blinded value

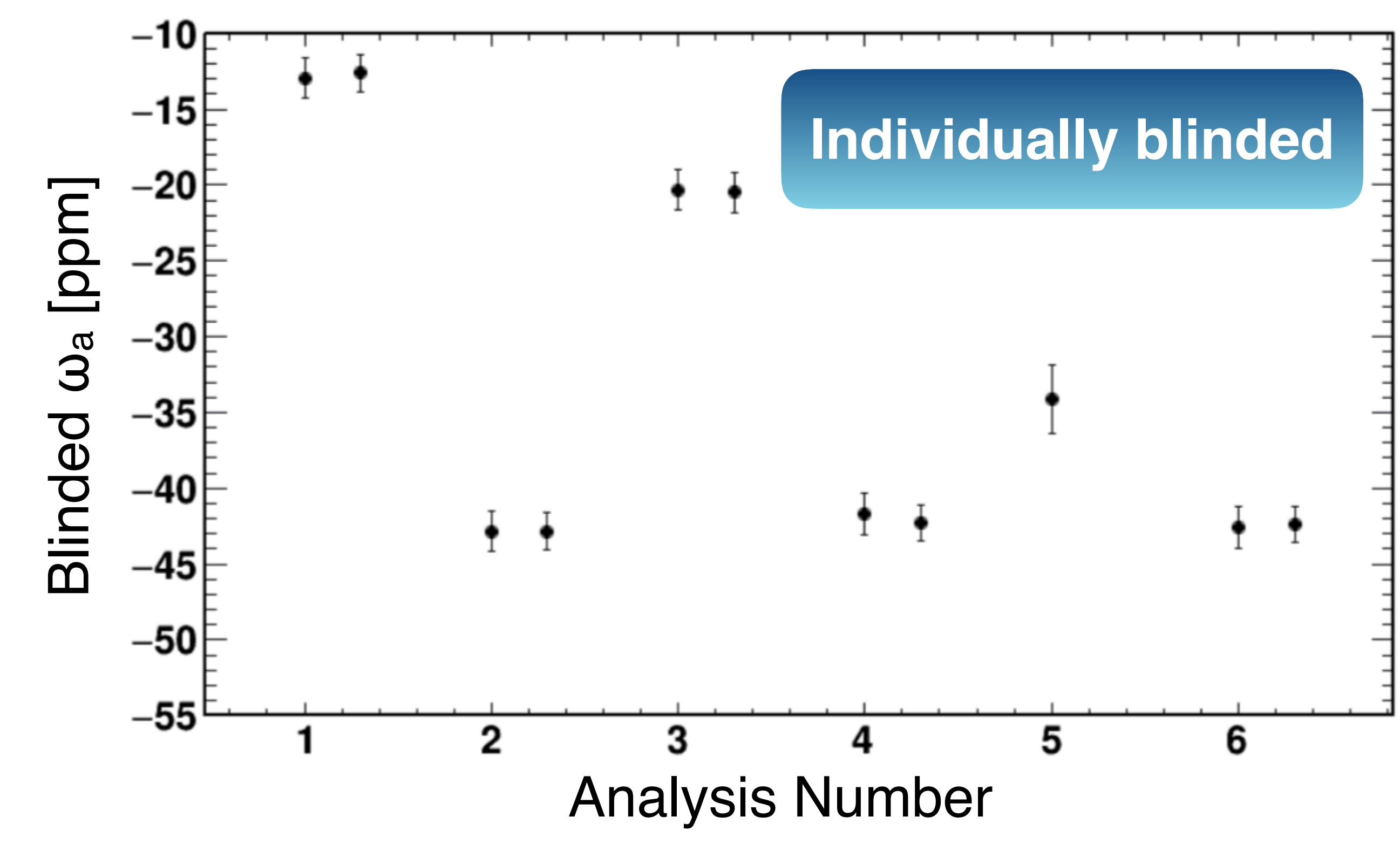




\section{Run 1 Analysis Status $-\omega_{p}$}

$$
a_{\mu}=\frac{\omega_{a}}{\sqrt[\tilde{\omega}_{p}]{\tilde{\omega}_{e}}} \frac{\mu_{p}}{\mu_{e}} \frac{m_{\mu}}{m_{e}} \frac{g_{e}}{2}
$$




\section{Run 1 Analysis Status: $\omega_{p}-$ Field Calibration}

- In the experiment, need to extract $\omega_{p}$; however, don't have free protons

- Need a calibration

- Field at the proton differs from the applied field

$\omega_{p}^{\text {meas }} \approx \omega_{p}^{\text {free }}$

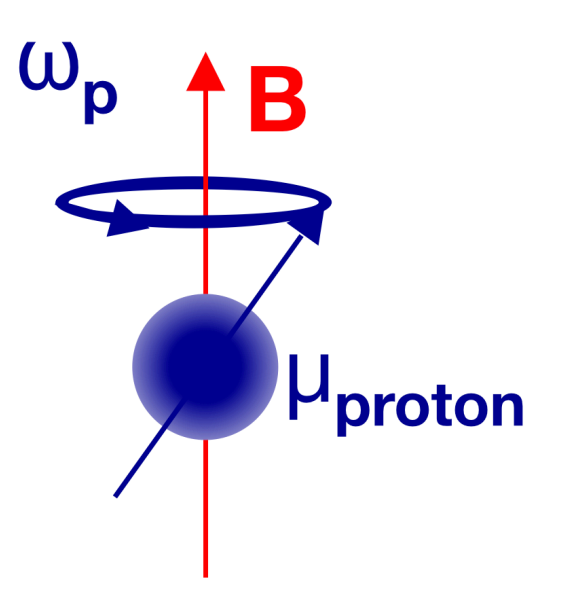
$\omega_{p}, \quad$ A
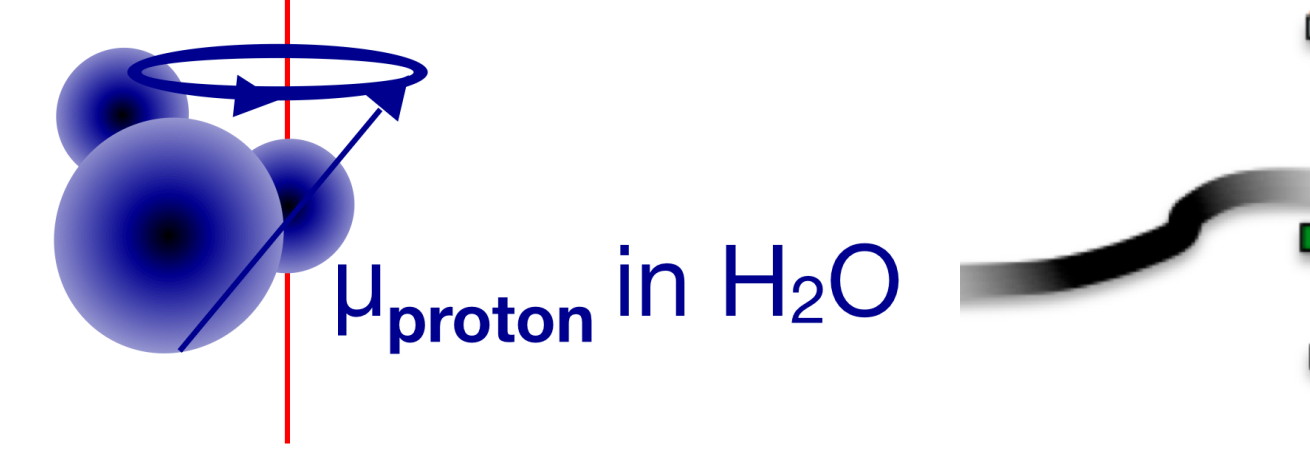


\section{Run 1 Analysis Status: $\omega_{p}-$ Field Calibration}

- In the experiment, need to extract $\omega_{\mathrm{p}} ; \quad \omega_{\mathrm{p}} \uparrow \mathbf{B} \quad \omega_{\mathrm{p}}, \uparrow \mathrm{B}$ however, don't have free protons

- Need a calibration

- Field at the proton differs from the

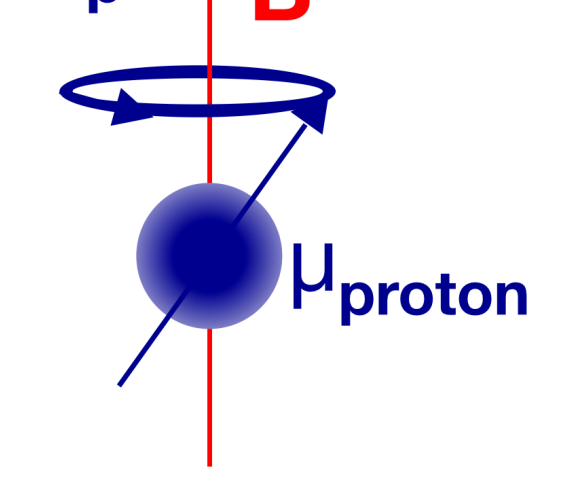
applied field

$\omega_{p}^{\text {meas }}=\omega_{p}^{\text {free }}\left[1-\sigma\left(\mathrm{H}_{2} \mathrm{O}, T\right)\right.$

Protons in $\mathrm{H}_{2} \mathrm{O}$ molecules, diamagnetism of electrons screens protons $=>$ local $\mathrm{B}$ changes

- $\sigma=25691(11) \times 10^{-9}$ at $25 \operatorname{deg}$ C [P.J. Mohr et al, Rev. Mod. Phys. 84, 1527 (2012)] 


\section{Run 1 Analysis Status: $\omega_{p}-$ Field Calibration}

- In the experiment, need to extract $\omega_{p} ; \omega_{p} \uparrow \mathbf{B} \quad \omega_{p}, \quad$ A however, don't have free protons

- Need a calibration

- Field at the proton differs from the
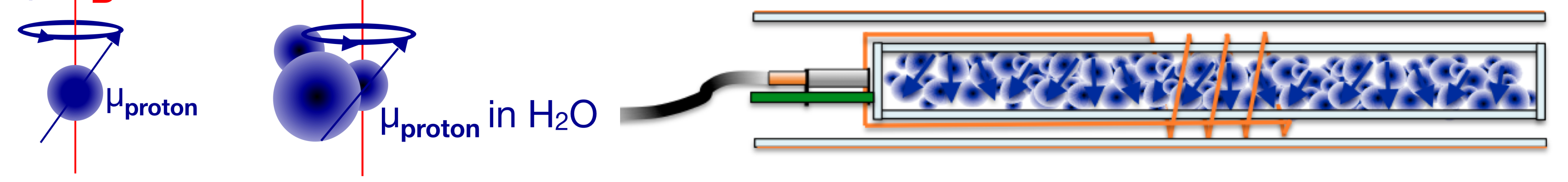
applied field

$\omega_{p}^{\text {meas }}=\omega_{p}^{\text {free }}[1-\underbrace{\sigma}\left(\mathrm{H}_{2} \mathrm{O}, T\right)-\left(\frac{\varepsilon}{4 \pi}-\frac{1}{3}\right) \chi\left(\mathrm{H}_{2} \mathrm{O}, T\right)$

Protons in $\mathrm{H}_{2} \mathrm{O}$ molecules, diamagnetism of electrons screens protons $=>$ local $B$ changes

- $\sigma=25691(11) \times 10^{-9}$ at $25 \mathrm{deg}$ C [P.J. Mohr et al, Rev. Mod. Phys. 84, 1527 (2012)]

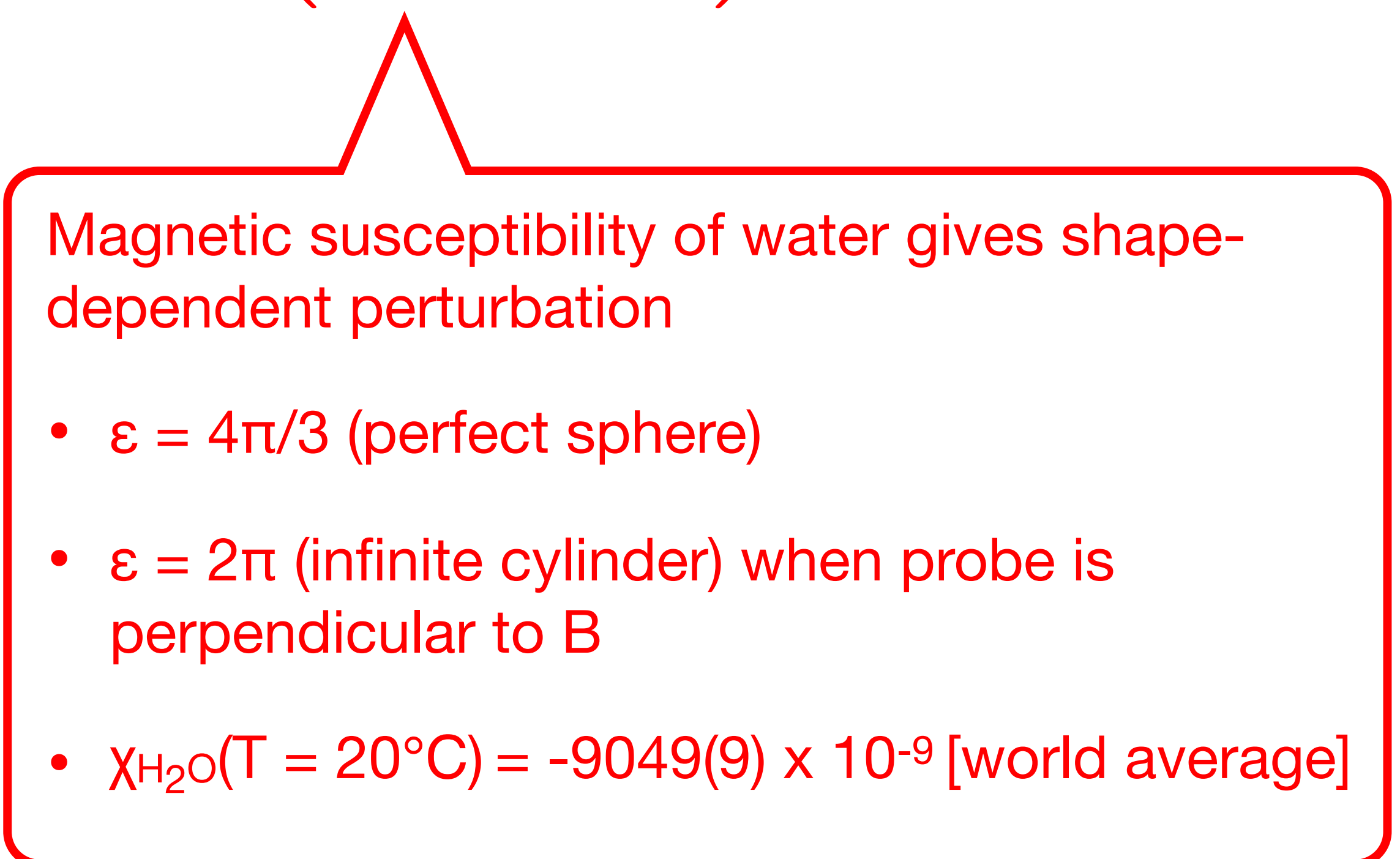




\section{Run 1 Analysis Status: $\omega_{p}-$ Field Calibration}

- In the experiment, need to extract $\omega_{p} ;$ however, don't have free protons

- Need a calibration

- Field at the proton differs from the

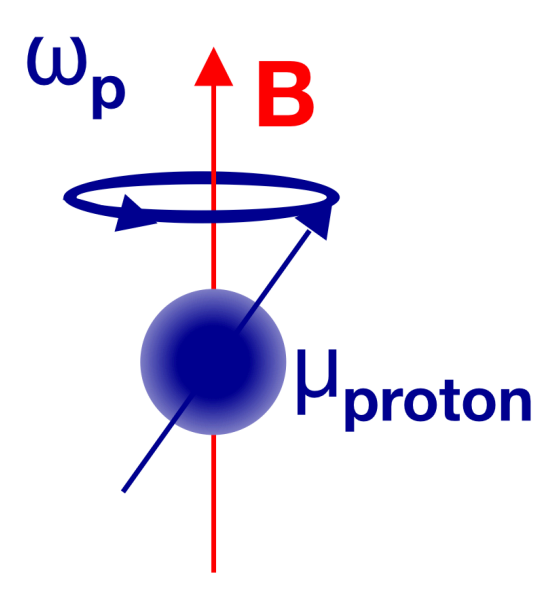
$\omega_{\mathbf{p}}, \quad \boldsymbol{A}$ applied field
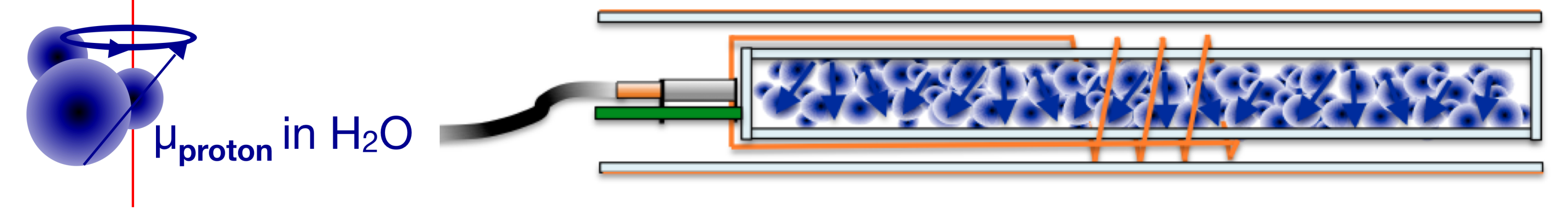

$\omega_{p}^{\text {meas }}=\omega_{p}^{\text {free }}[1-\underbrace{\sigma}\left(\mathrm{H}_{2} \mathrm{O}, T\right)-\left(\frac{\varepsilon}{4 \pi}-\frac{1}{3}\right) \chi\left(\mathrm{H}_{2} \mathrm{O}, T\right)-\delta_{s}$

Protons in $\mathrm{H}_{2} \mathrm{O}$ molecules, diamagnetism of electrons screens protons $=>$ local $B$ changes

- $\sigma=25691(11) \times 10^{-9}$ at $25 \operatorname{deg}$ C [P.J. Mohr et al, Rev. Mod. Phys. 84, 1527 (2012)]

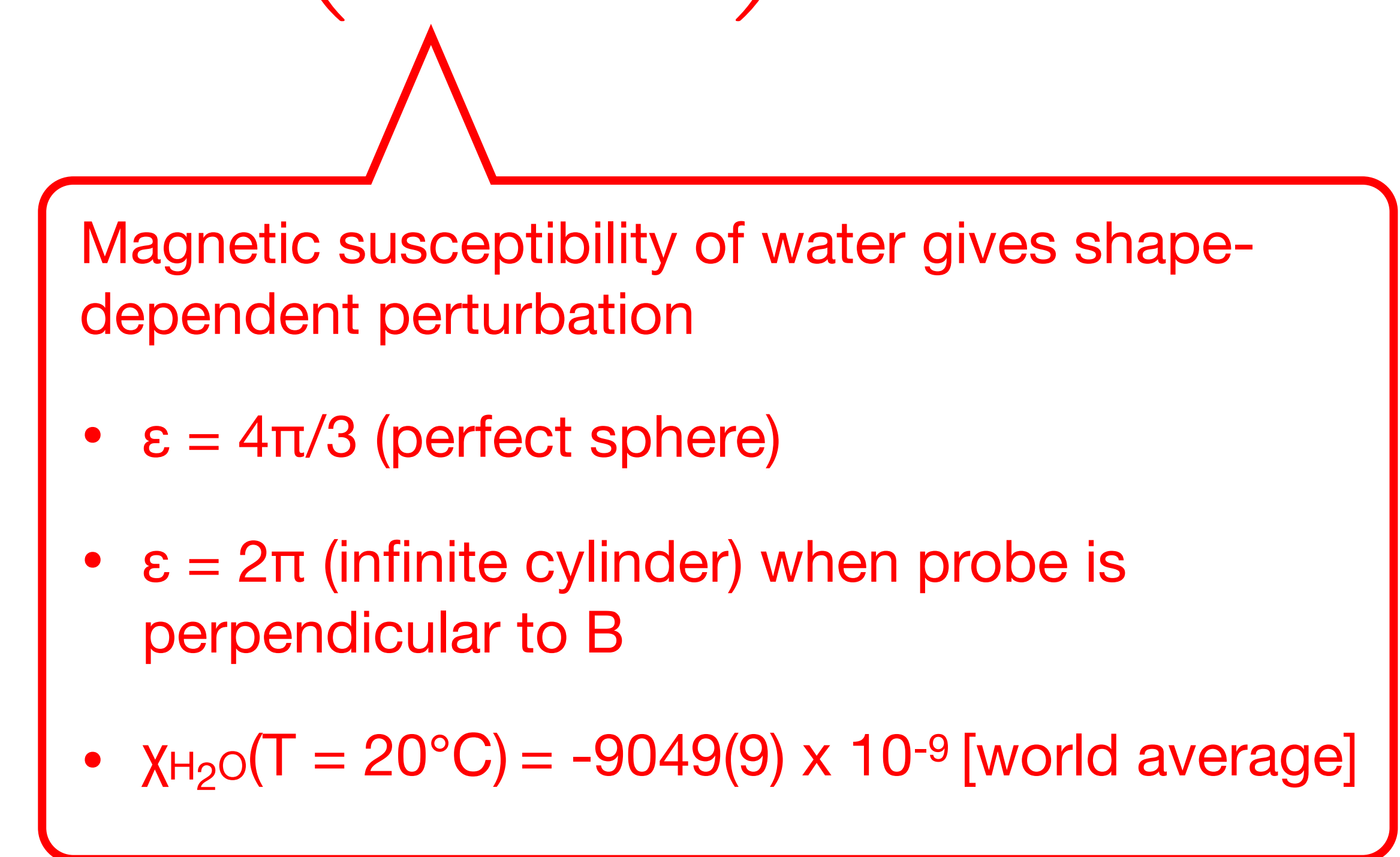

Magnetization of probe materials, geometry perturbs field experienced by protons 


\section{Run 1 Analysis Status: $\omega_{p}-$ Field Calibration}

- In the experiment, need to extract $\omega_{p}$; however, don't have free protons

- Need a calibration

- Field at the proton differs from the applied field

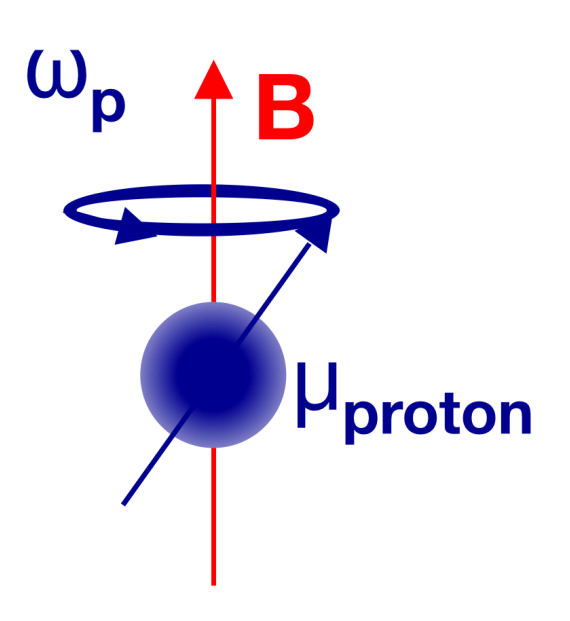

$\omega_{p}, \quad \hat{T} \mathbf{B}$
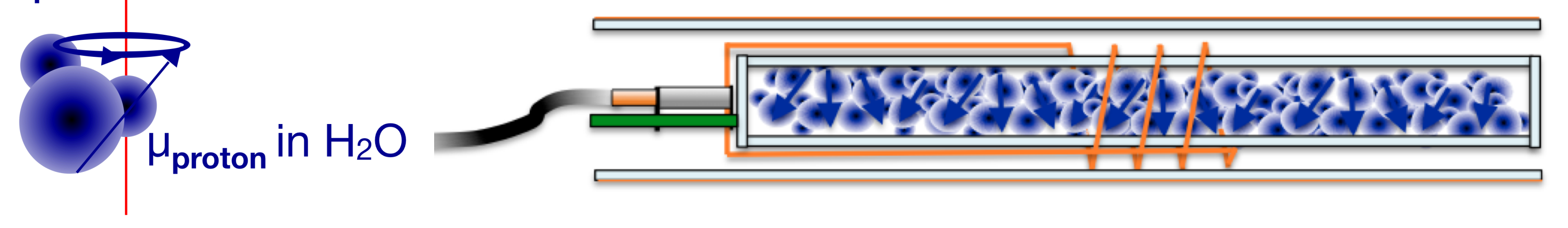

paramagnetic impurities in water sample

$\omega_{p}^{\text {meas }}=\omega_{p}^{\text {free }}[1-\underbrace{\sigma}\left(\mathrm{H}_{2} \mathrm{O}, T\right)-\left(\frac{\varepsilon}{4 \pi}-\frac{1}{3}\right) \chi\left(\mathrm{H}_{2} \mathrm{O}, T\right)-\delta_{s}-\delta_{p}$

Protons in $\mathrm{H}_{2} \mathrm{O}$ molecules, diamagnetism of electrons screens protons $=>$ local $B$ changes

- $\sigma=25691(11) \times 10^{-9}$ at $25 \operatorname{deg}$ C [P.J. Mohr et al, Rev. Mod. Phys. 84, 1527 (2012)]

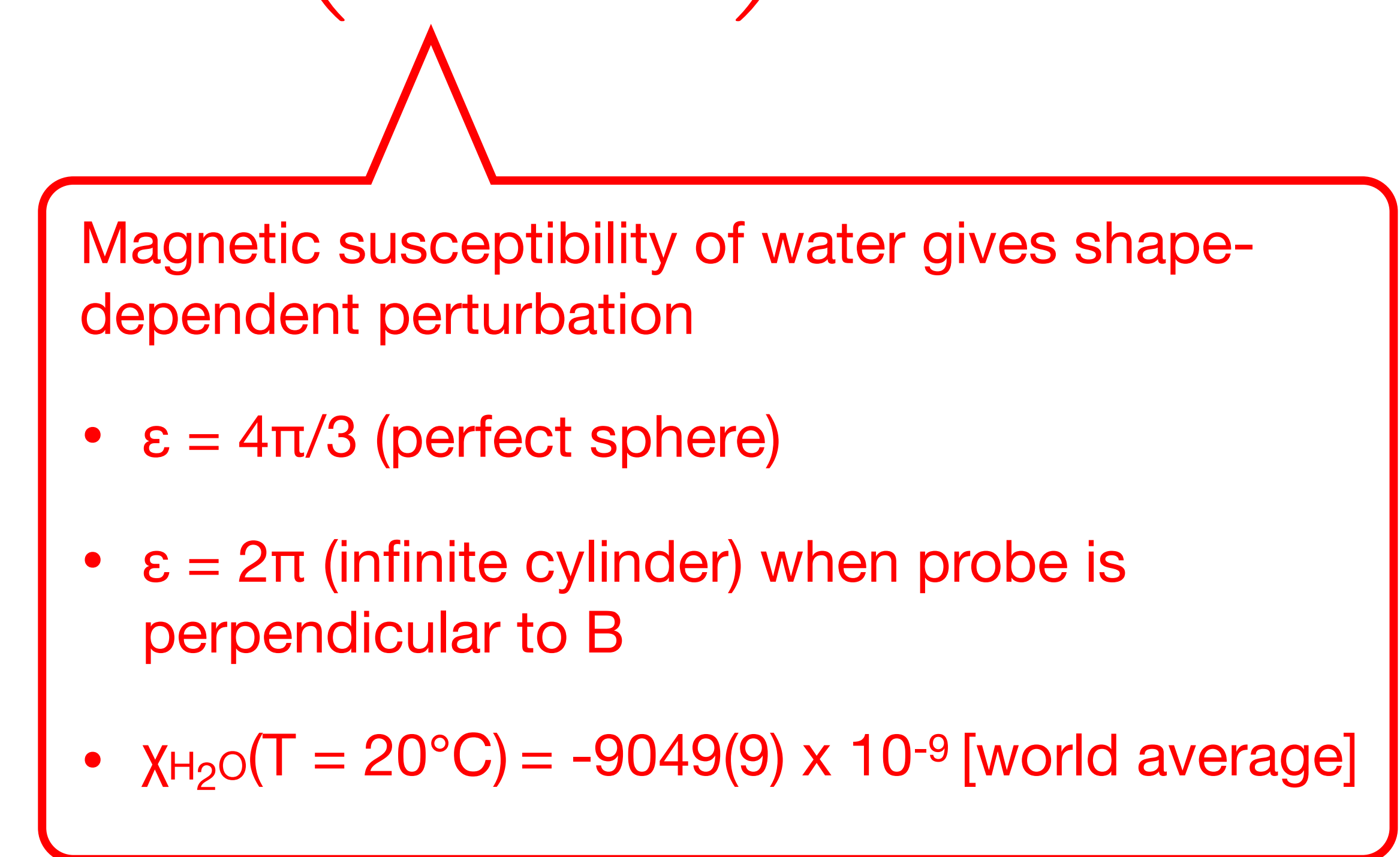




\section{Run 1 Analysis Status: $\omega_{p}-$ Field Calibration}

- In the experiment, need to extract $\omega_{p}$; however, don't have free protons

- Need a calibration

- Field at the proton differs from the applied field

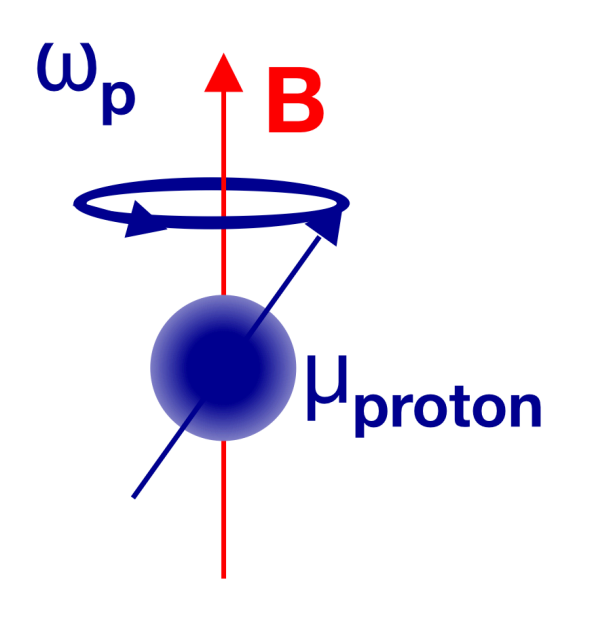

$\omega_{p}, \quad \hat{T} B$
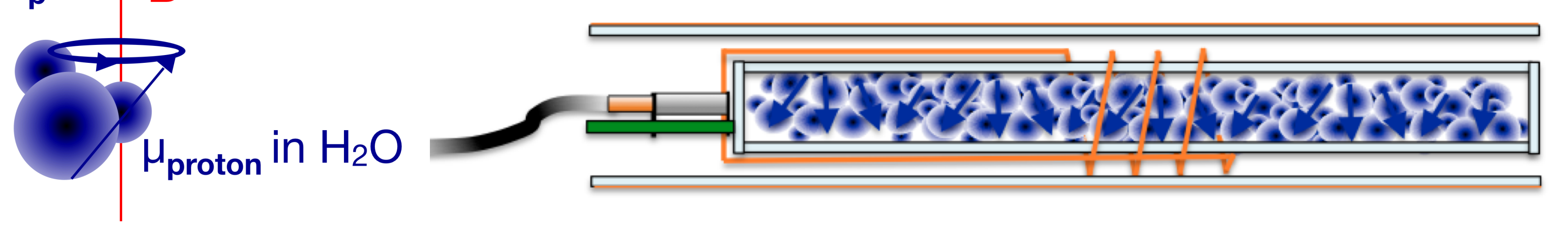

paramagnetic impurities in water sample

$\omega_{p}^{\text {meas }}=\omega_{p}^{\text {free }}[1-\underbrace{\sigma}\left(\mathrm{H}_{2} \mathrm{O}, T\right)-\left(\frac{\varepsilon}{4 \pi}-\frac{1}{3}\right) \chi\left(\mathrm{H}_{2} \mathrm{O}, T\right)-\delta_{s}-\delta_{p}-\delta_{\mathrm{RD}}-\delta_{d}]$

Protons in $\mathrm{H}_{2} \mathrm{O}$ molecules, diamagnetism of electrons screens protons $=>$ local $B$ changes

- $\sigma=25691(11) \times 10^{-9}$ at $25 \operatorname{deg}$ C [P.J. Mohr et al, Rev. Mod. Phys. 84, 1527 (2012)]

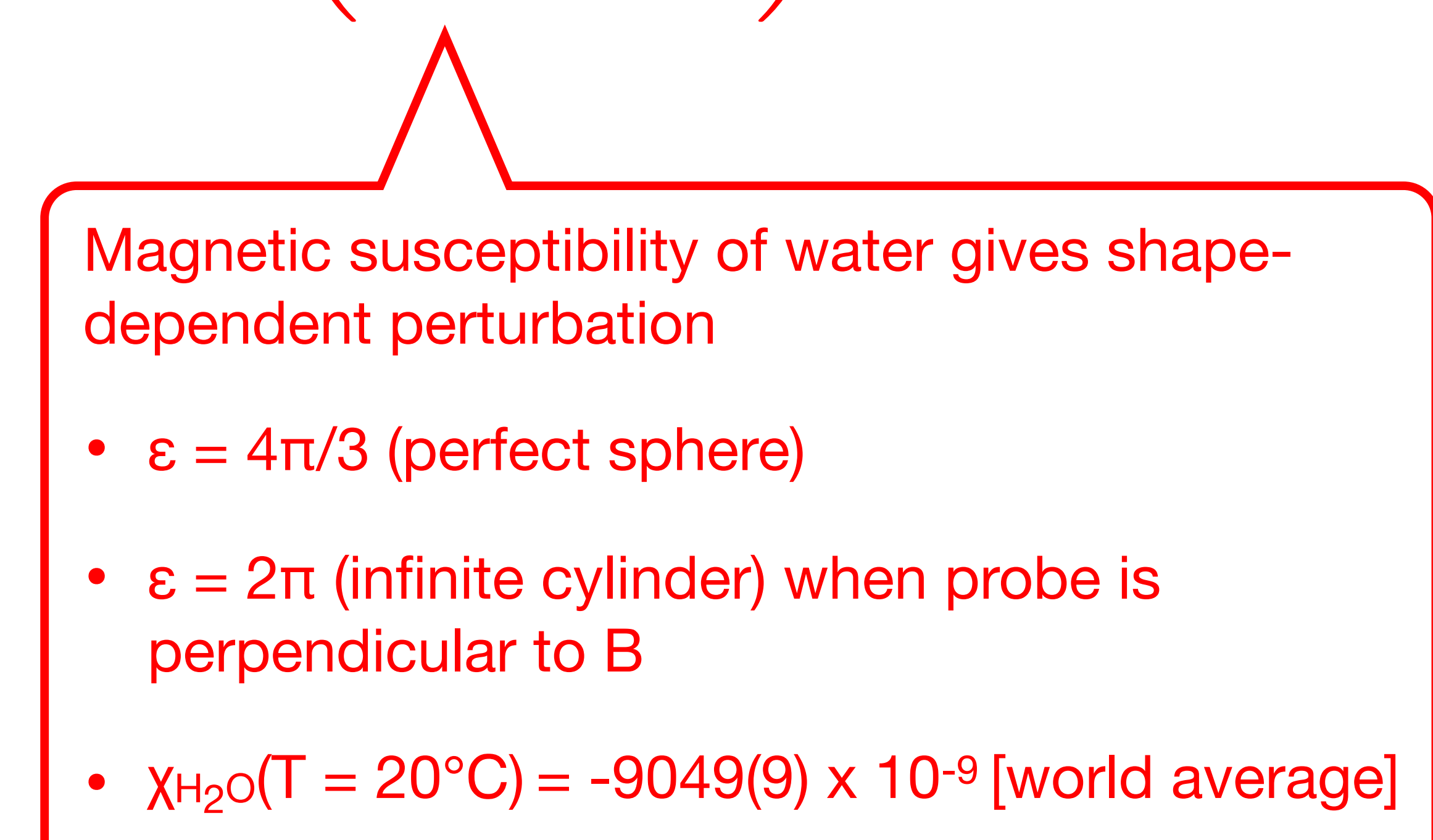

Magnetization of probe materials, geometry perturbs field experienced by protons

Dynamic effects: radiation damping, dipolar field from protons 


\section{Run 1 Analysis Status: $\omega_{p}-$ Field Calibration}

- In the experiment, need to extract $\omega_{p}$; however, don't have free protons

- Need a calibration

- Field at the proton differs from the applied field

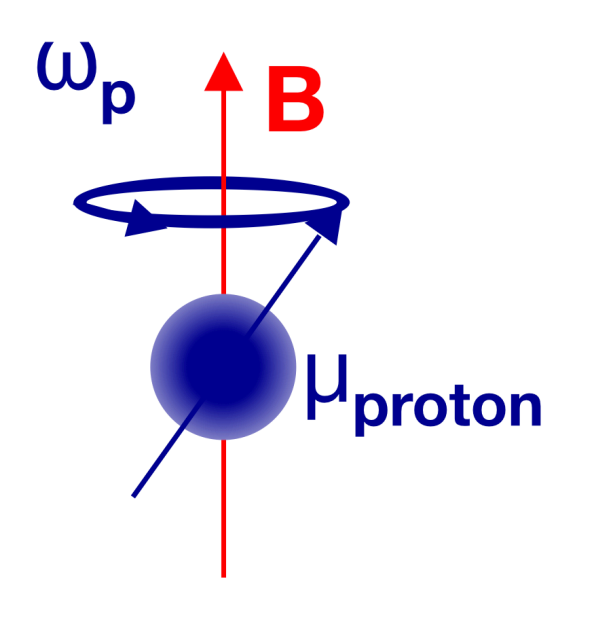

$\omega_{p}, \quad \hat{T} B$
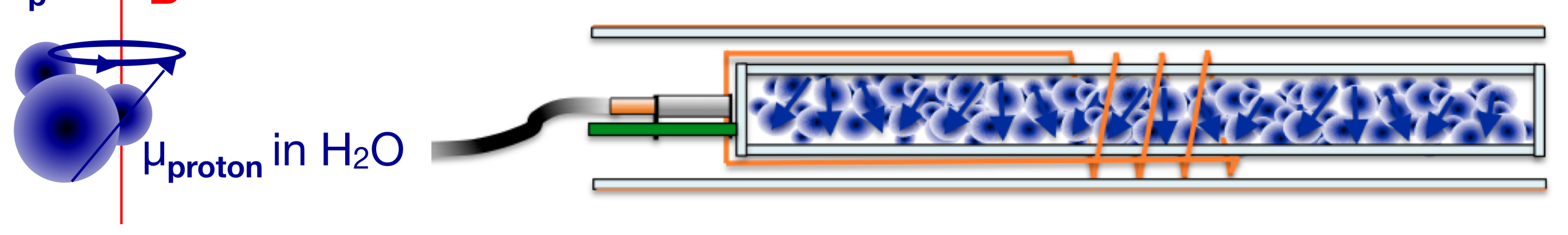

paramagnetic impurities in water sample

$\omega_{p}^{\text {meas }}=\omega_{p}^{\text {free }}\left[1-\sigma\left(\mathrm{H}_{2} \mathrm{O}, T\right)-\left(\frac{\varepsilon}{4 \pi}-\frac{1}{3}\right) \chi\left(\mathrm{H}_{2} \mathrm{O}, T\right)-\delta_{s}-\delta_{p}-\delta_{\mathrm{RD}}-\delta_{d}\right]$

Protons in $\mathrm{H}_{2} \mathrm{O}$ molecules, diamagnetism of electrons screens protons $=>$ local $\mathrm{B}$ changes

- $\sigma=25691(11) \times 10^{-9}$ at $25 \mathrm{deg}$ C [P.J. Mohr et al, Rev. Mod. Phys. 84, 1527 (2012)]

Goal: Determine total correction to $\leq 35 \mathrm{ppb}$ accuracy

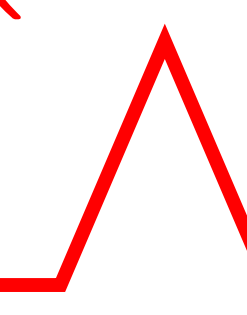

Magnetic susceptibility of water gives shapedependent perturbation

- $\varepsilon=4 \pi / 3$ (perfect sphere)

- $\varepsilon=2 \pi$ (infinite cylinder) when probe is perpendicular to $B$

- $\mathrm{X}_{2} \mathrm{O}\left(\mathrm{T}=20^{\circ} \mathrm{C}\right)=-9049(9) \times 10^{-9}[$ world average $]$

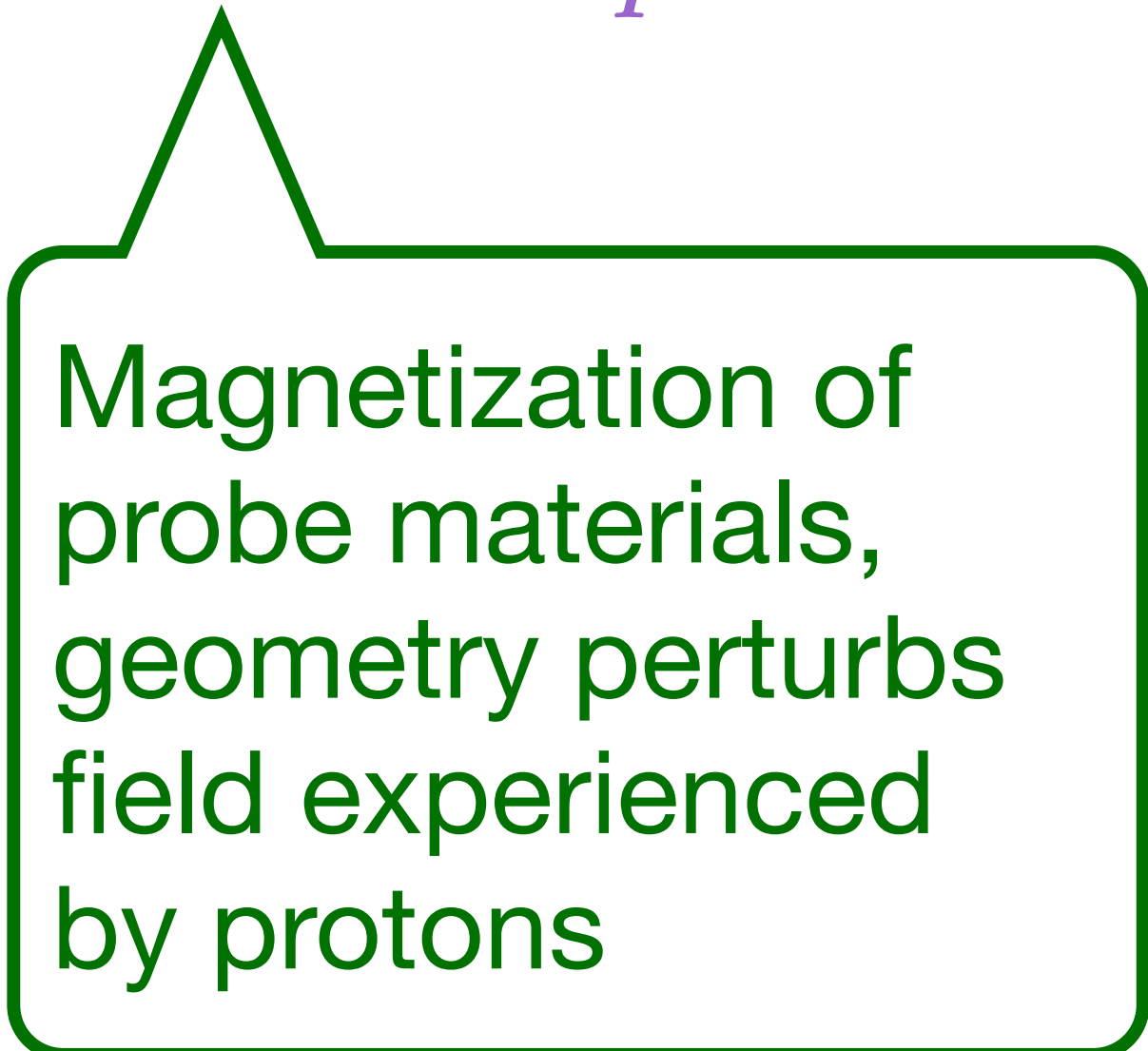

Dynamic effects: radiation damping, dipolar field from protons 


\section{Run 1 Analysis Status: $\omega_{p}-$ Field Calibration}

\section{Plunging Probe}

- Achieved small perturbation of plunging probe $\left(\delta_{s}+\delta_{p}+\delta_{R D}+\delta_{d}\right):(-0.2 \pm 11.4) p p b$

- Quantified uncertainties on plunging probe material, dynamic effects - under budget of $35 \mathrm{ppb}$ by a factor of $>2$

\begin{tabular}{|ccc|}
\hline \multicolumn{2}{|c|}{ Plunging Probe Perturbations } \\
Quantity & Symbol & Uncertainty $(\mathrm{ppb})$ \\
\hline Material Perturbation & $\delta_{\mathrm{s}}$ & 10.9 \\
Paramagnetic Impurities & $\delta_{\mathrm{p}}$ & 1.1 \\
Radiation Damping & $\delta_{\mathrm{RD}}$ & 2 \\
Proton Dipolar Fields & $\delta_{\mathrm{d}}$ & 2.3 \\
Bulk Magnetic Susceptibility & $\delta_{\mathrm{b}}$ & 2 \\
Water Diamagnetic Shielding & $\sigma$ & 11 \\
\hline TOTAL & & $\mathbf{1 5 . 9}$ \\
\hline
\end{tabular}




\section{Run 1 Analysis Status: $\omega_{p}-$ Field Calibration}

\section{Plunging Probe}

- Achieved small perturbation of plunging probe $\left(\delta_{s}+\delta_{p}+\delta_{R D}+\delta_{d}\right):(-0.2 \pm 11.4) p p b$

- Quantified uncertainties on plunging probe material, dynamic effects - under budget of $35 \mathrm{ppb}$ by a factor of $>2$

\begin{tabular}{|c|c|c|}
\hline \multicolumn{3}{|c|}{ Plunging Probe Perturbations } \\
\hline Quantity & Symbol & Uncertainty (ppb) \\
\hline Material Perturbation & $\delta$ & 10.9 \\
\hline Paramagnetic Impurities & $\delta_{p}$ & 1.1 \\
\hline Radiation Damping & $\delta_{\mathrm{RD}}$ & 2 \\
\hline Proton Dipolar Fields & $\delta_{d}$ & 2.3 \\
\hline Bulk Magnetic Susceptibility & $\delta_{b}$ & 2 \\
\hline Water Diamagnetic Shielding & $\sigma$ & 11 \\
\hline TOTAL & & 15.9 \\
\hline
\end{tabular}

Trolley Calibration

- Calibration of trolley probes under control

- Factor of $\geq 2$ improvement on uncertainties for nearly all probes compared to E821

- Uncertainty is $<\sim 40 \mathrm{ppb}$ on average per probe - on budget

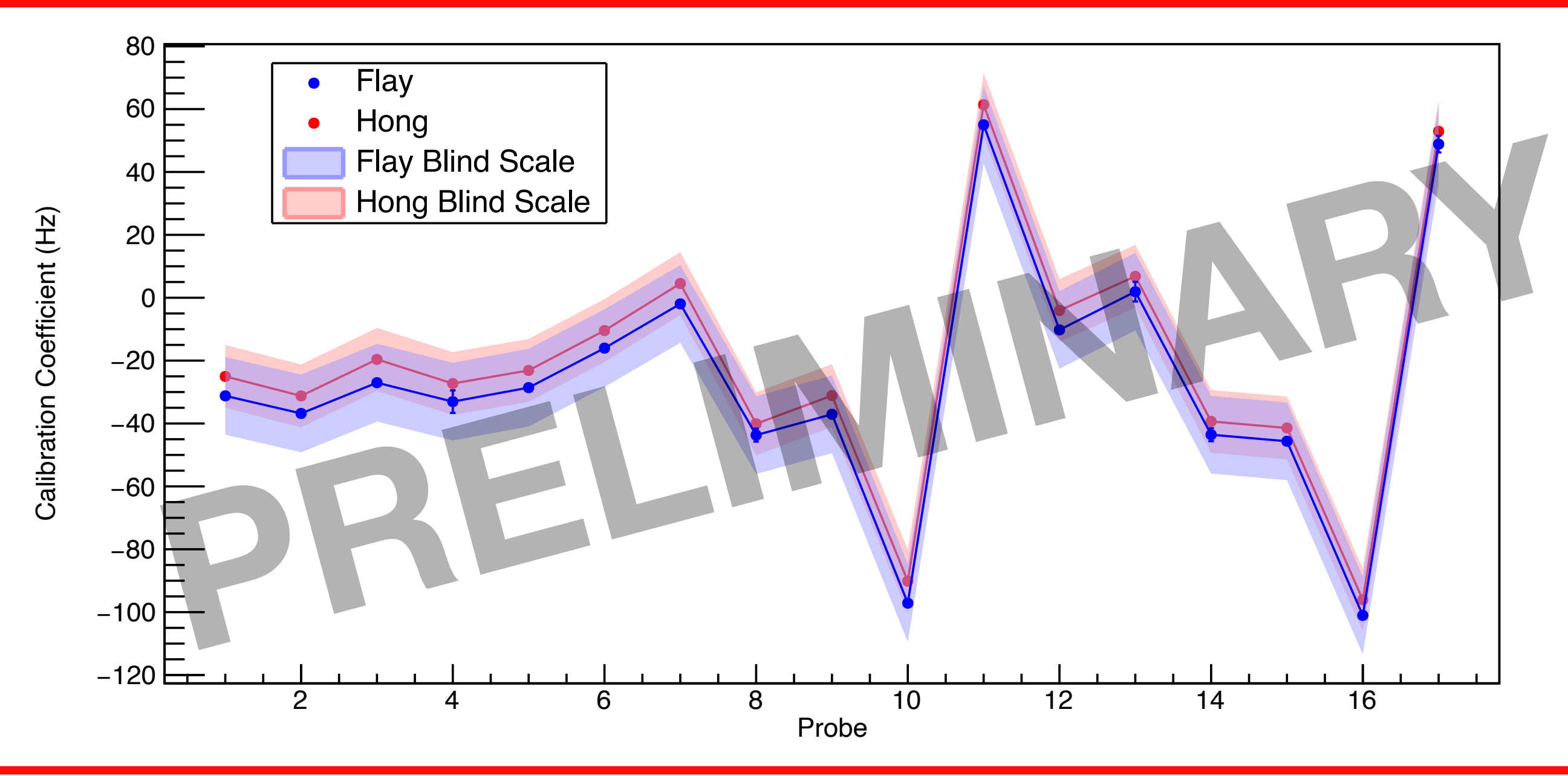

UMassAmherst 


\section{Run 1 Analysis Status: $\omega_{p}-$ Field Interpolation}

- Need to determine $\omega_{p}$ at all times while storing muons $=>$ interpolate between trolley maps using fixed probe data

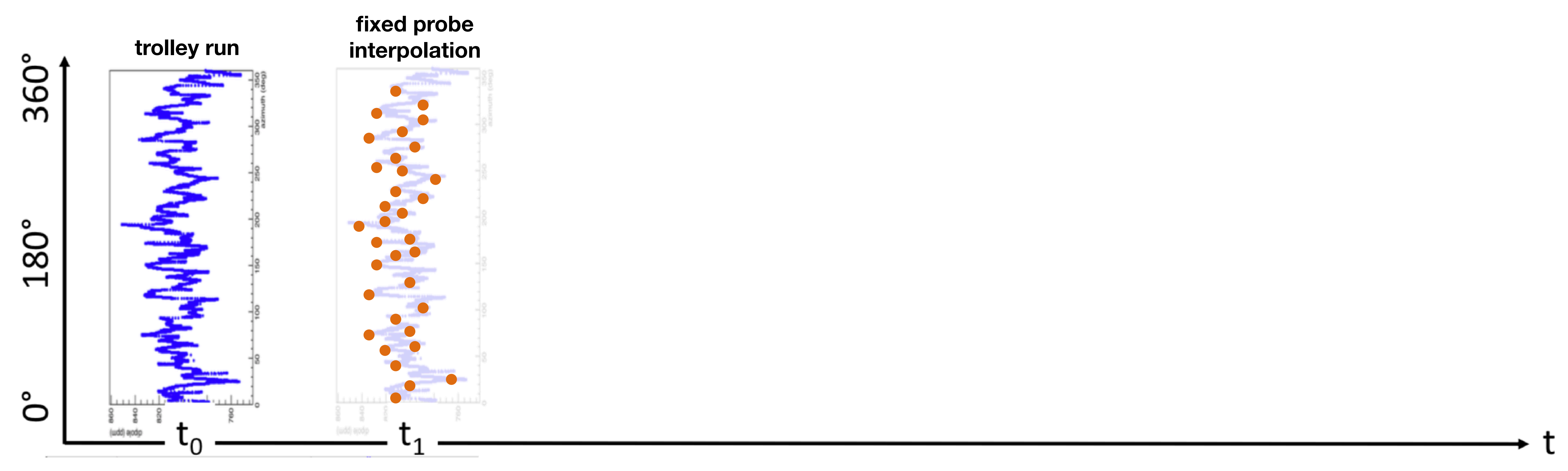




\section{Run 1 Analysis Status: $\omega_{p}-$ Field Interpolation}

- Need to determine $\omega_{p}$ at all times while storing muons $=>$ interpolate between trolley maps using fixed probe data

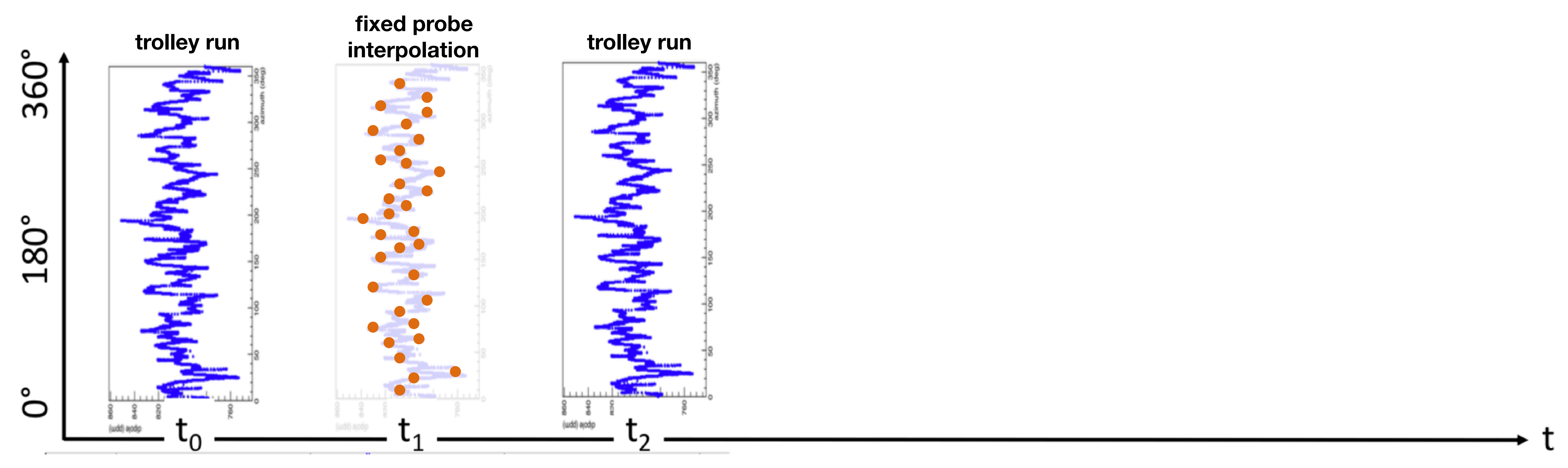




\section{Run 1 Analysis Status: $\omega_{p}-$ Field Interpolation}

- Need to determine $\omega_{p}$ at all times while storing muons $=>$ interpolate between trolley maps using fixed probe data

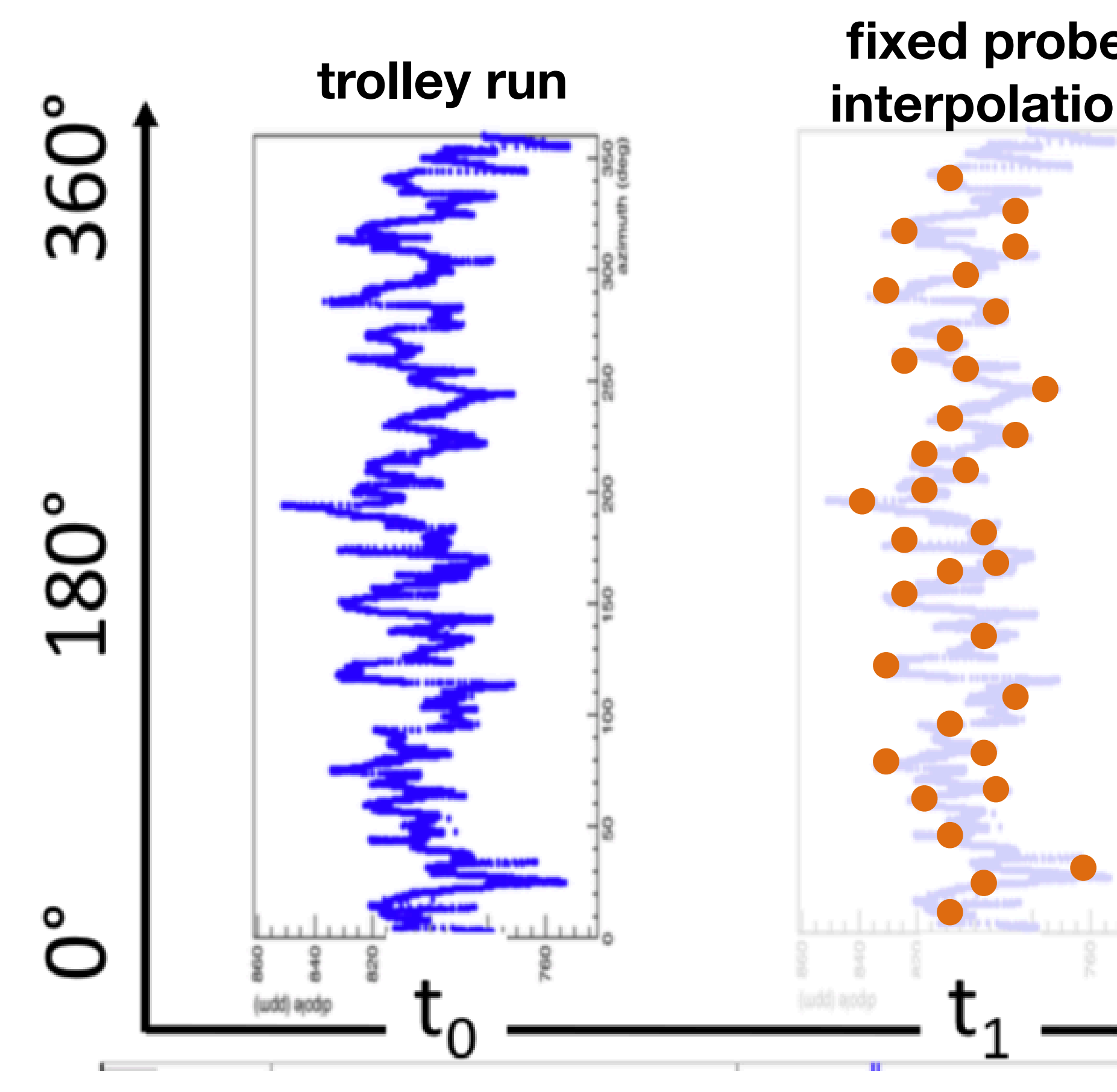

fixed probe

interpolation

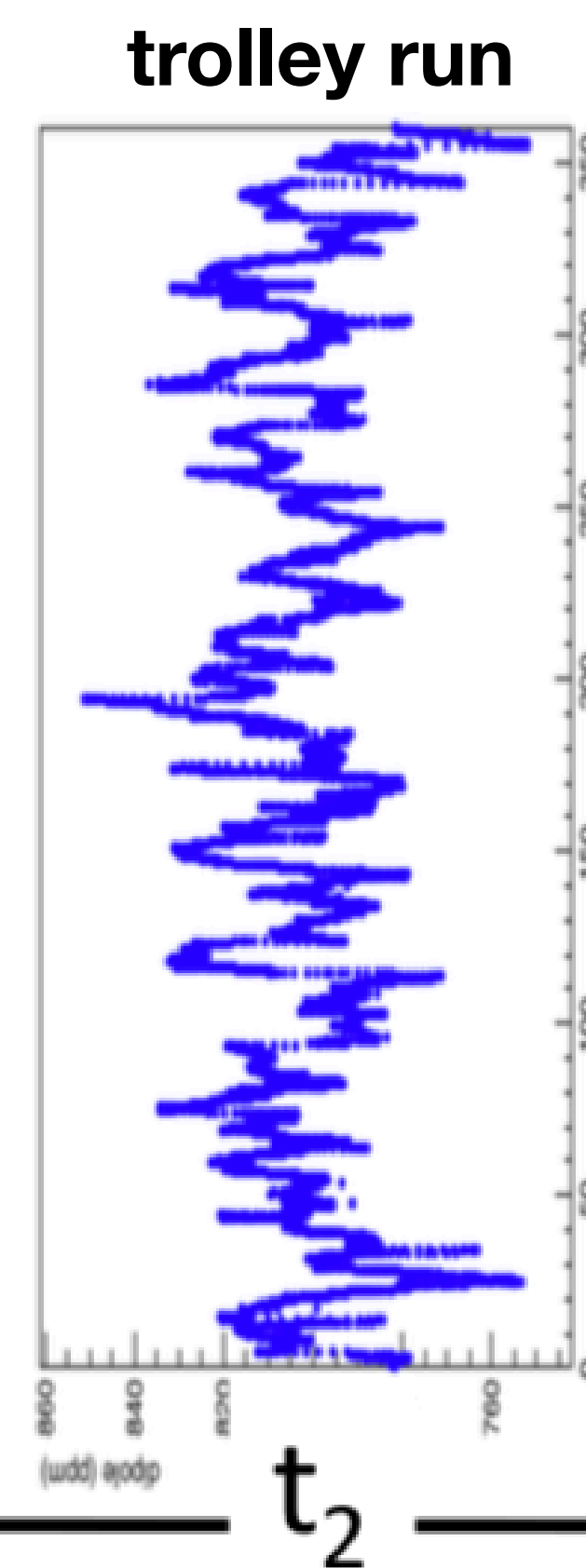

fixed probe

interpolation

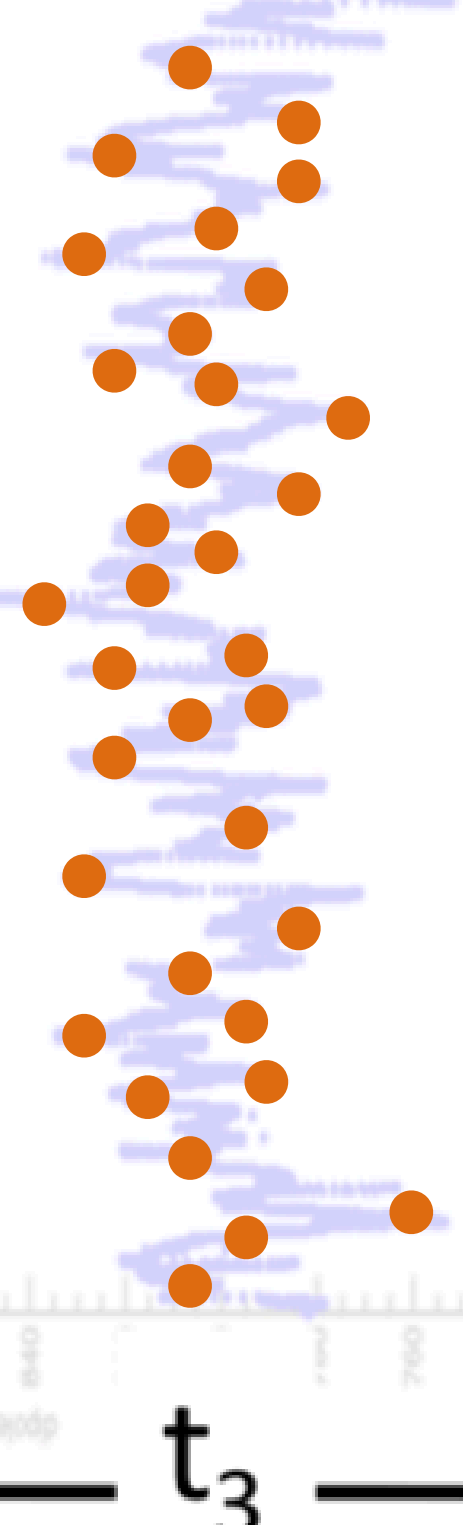




\section{Run 1 Analysis Status: $\omega_{p}-$ Field Interpolation}

- Example from subset of data

- Tracking algorithms showing good agreement with trolley runs

- Also tracking higher-order multipole moments important for extracting muon-weighted field $\tilde{\omega}_{p}$

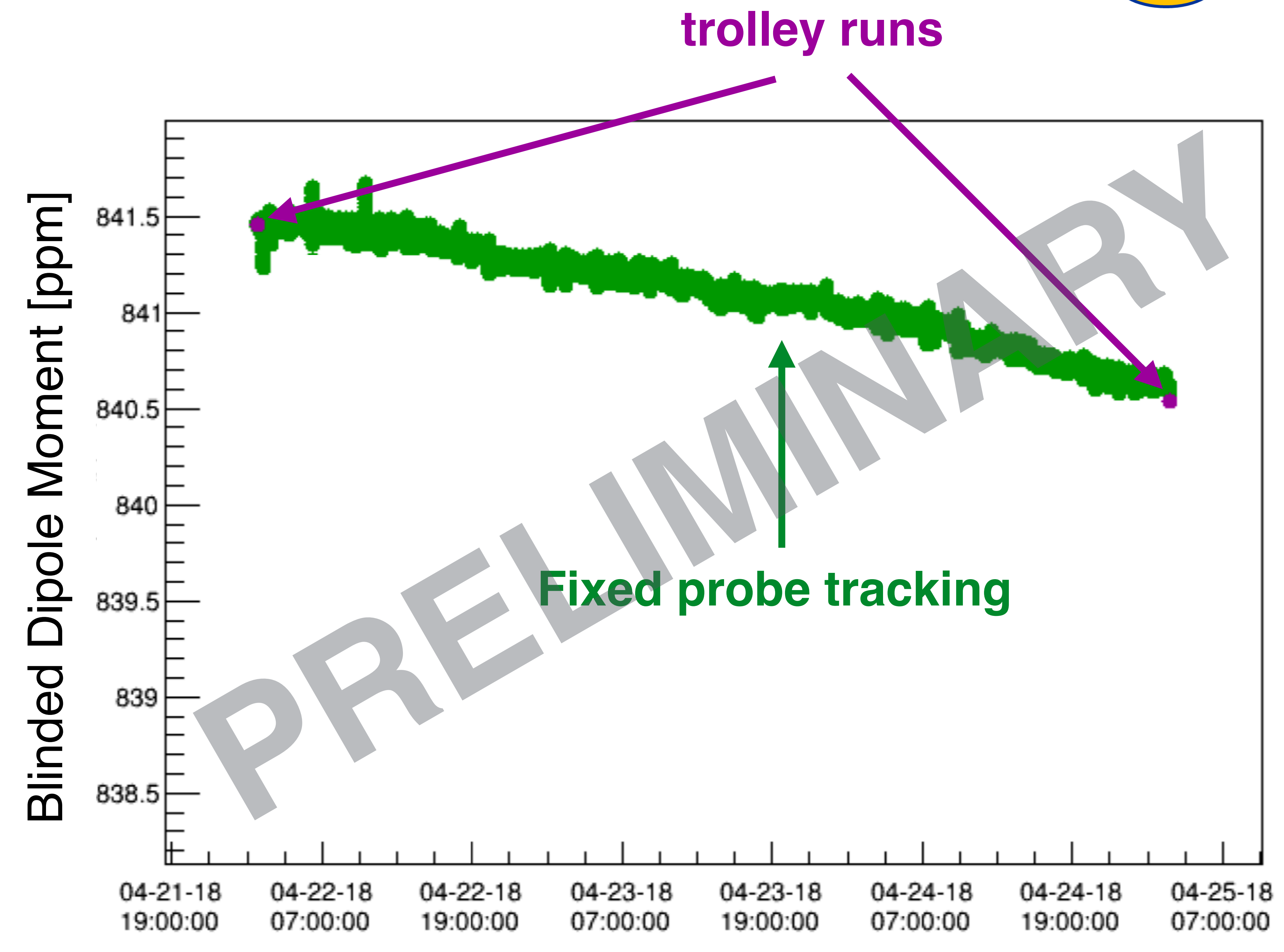




\section{Summary}

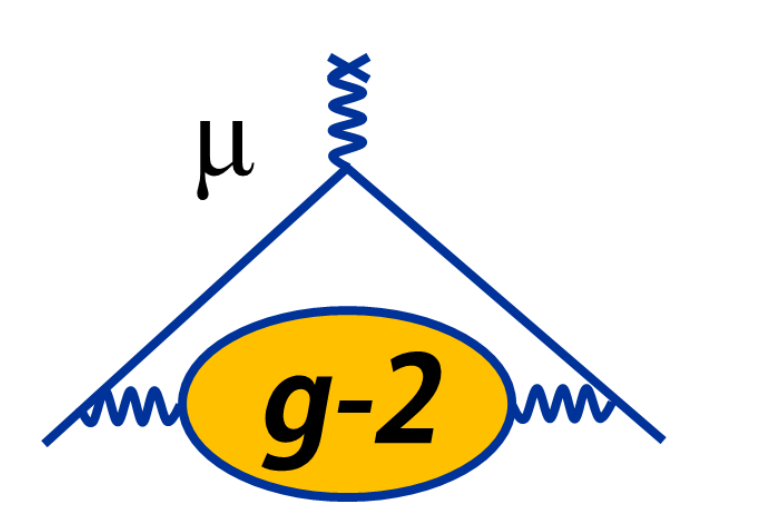

- The Muon g-2 Experiment is a highly sensitive test of the SM

- Discrepancy between theory and experiment for $a_{\mu}>\sim 3 \sigma$

Completed Run 1 in July 2018 (1.1x BNL statistics)

Analyses are mature and progressing towards a result in early 2020

Completed Run 2 in July 2019 (1.9x BNL statistics)

- Starting to organize analysis efforts

- Run 3 starting this November: aiming to triple statistics to date 


\section{Thank You!}

tor

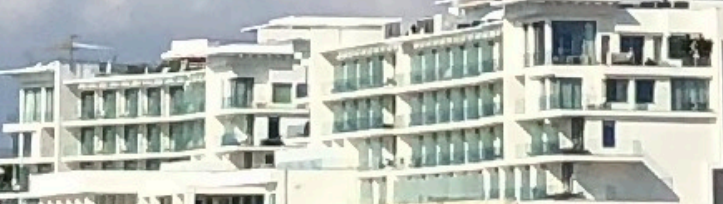

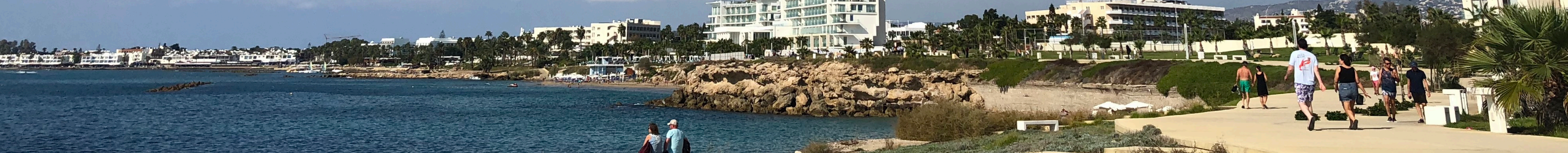

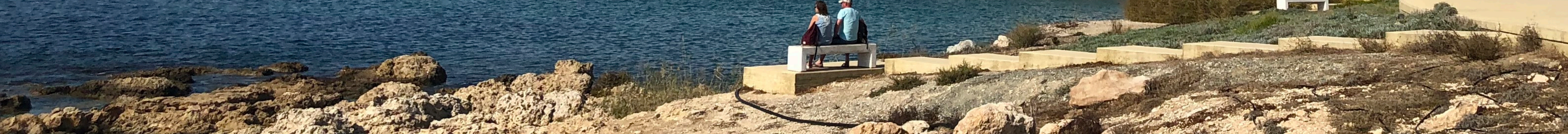

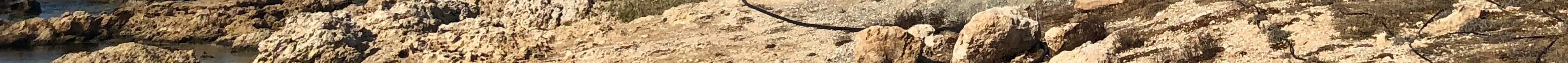

6.

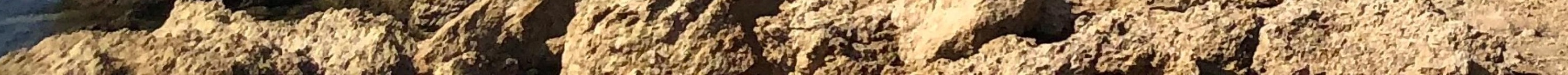

re 3 - 


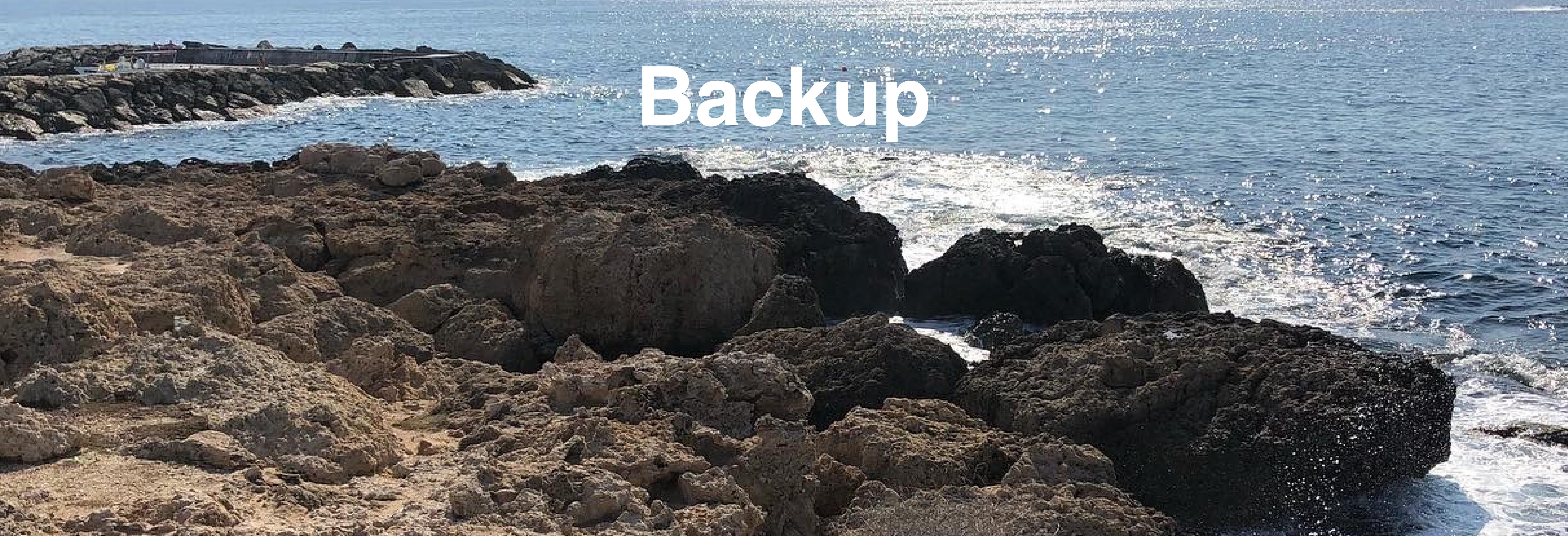




\section{The Big Move: Transporting the Ring from BNL to FNAL}

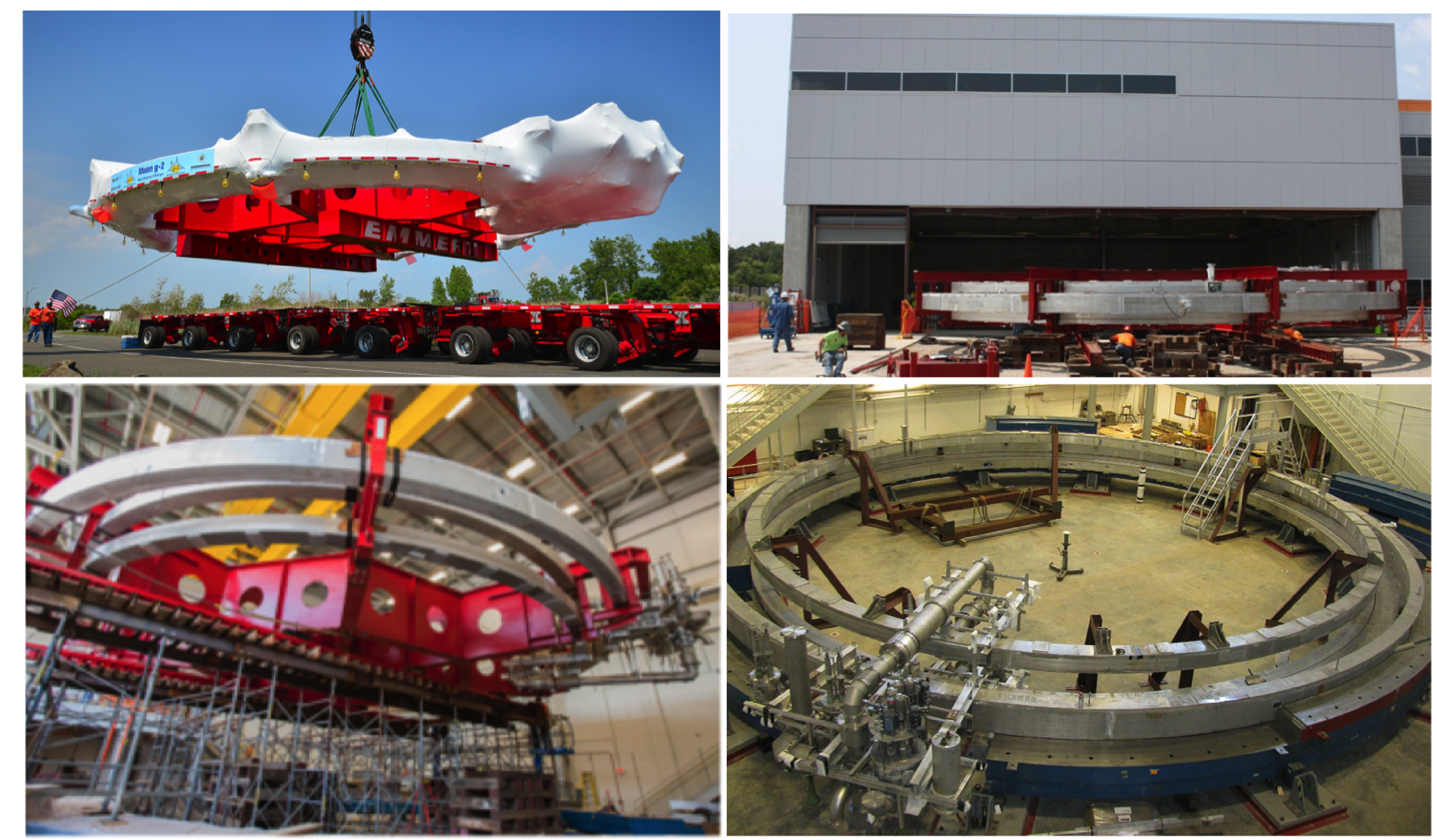

- June 2013-June 2015

- Ring deconstructed at BNL, transported by barge/ flatbed trailer

- Reassembled at FNAL

- Ring successfully cooled and powered to $1.45 \mathrm{~T}$ in September 2015 remarkable achievement! 


\section{Getting Muons Into the Ring: Inflector Magnet}

- Outside ring: $\mathrm{B}=0 \mathrm{~T}$, inside: $\mathrm{B}=1.45 \mathrm{~T}$

- Need to cancel field in order to get muons in (strong deflection otherwise)

- No perturbation to field outside shield

- New inflector design with higher transmission under development

- Improve injection by $\mathbf{4 0 \%}$

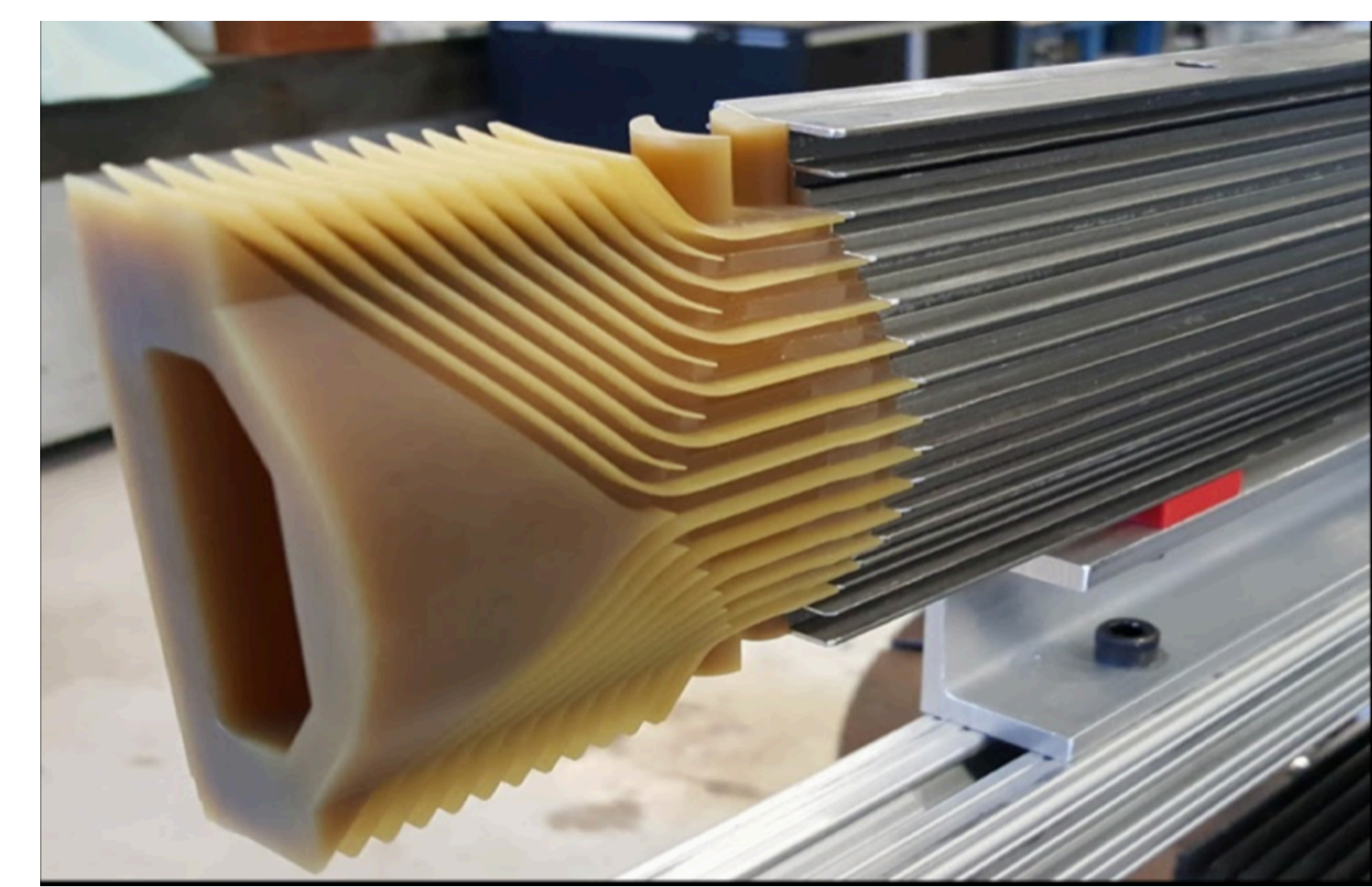

New inflector coil winding mount

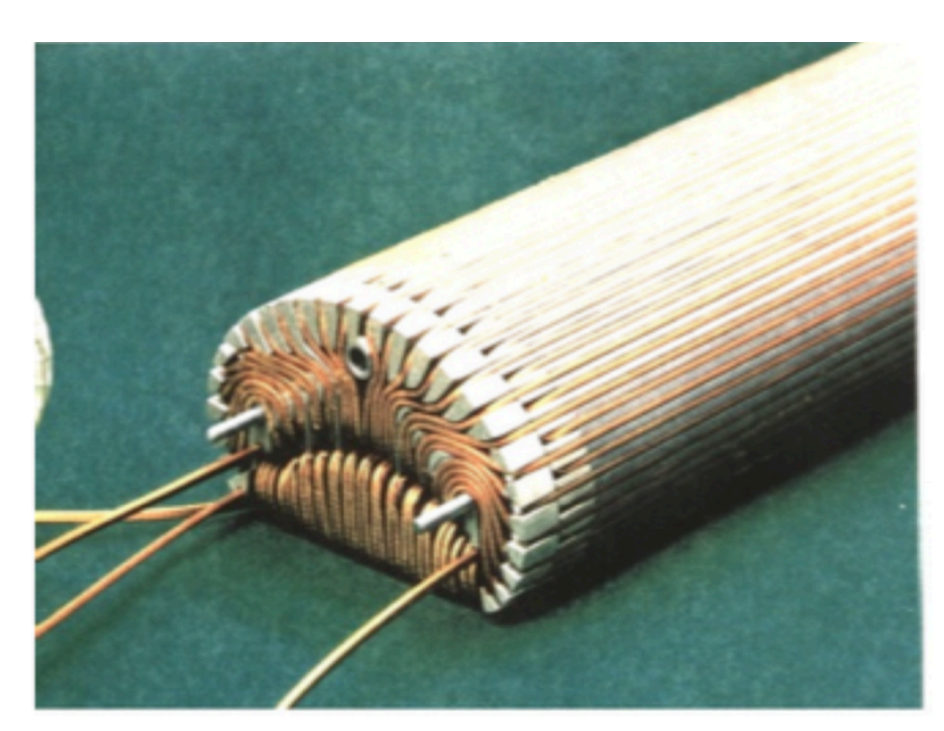

Present inflector

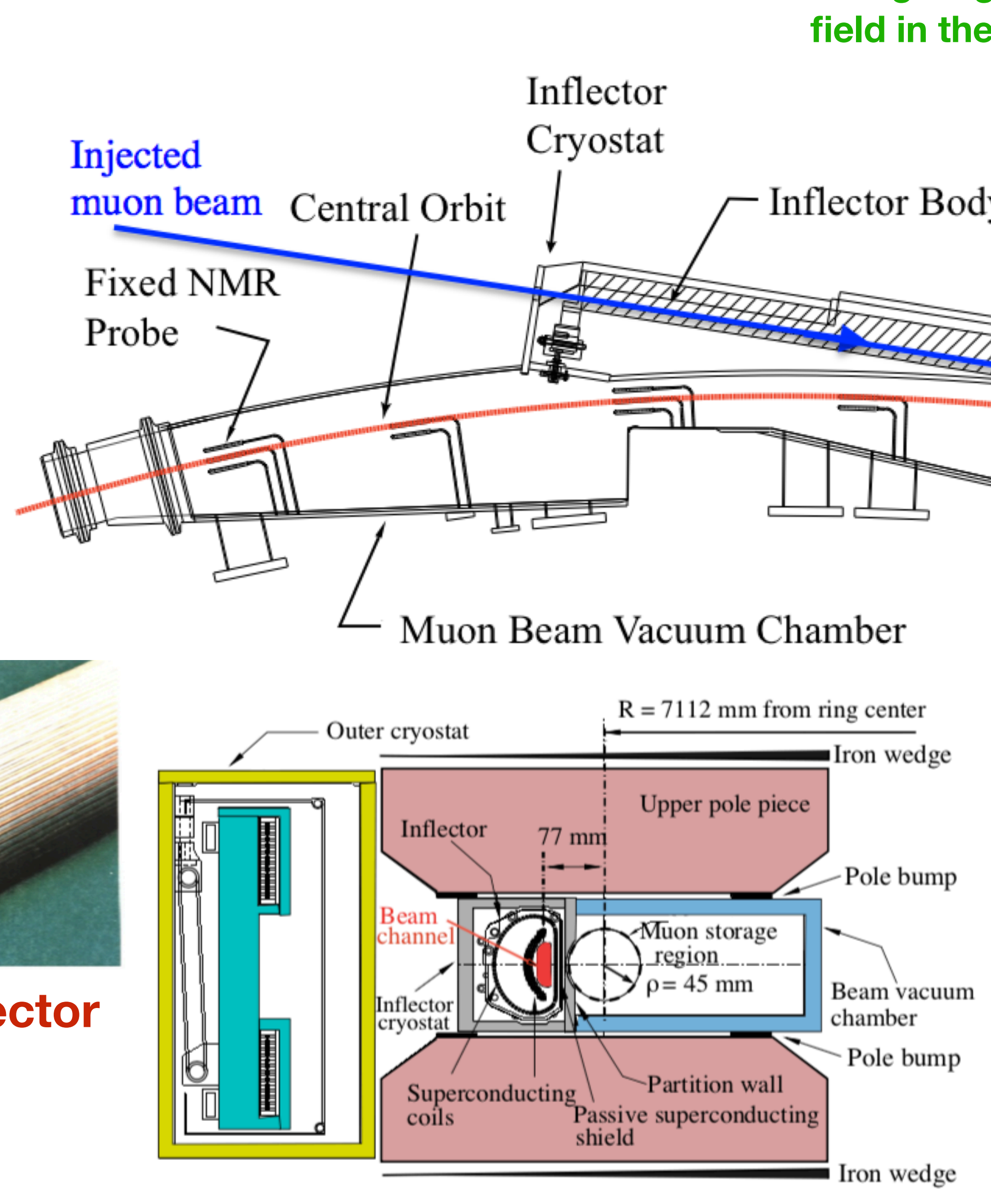

Super currents in passive sup shield prevents flux leakage
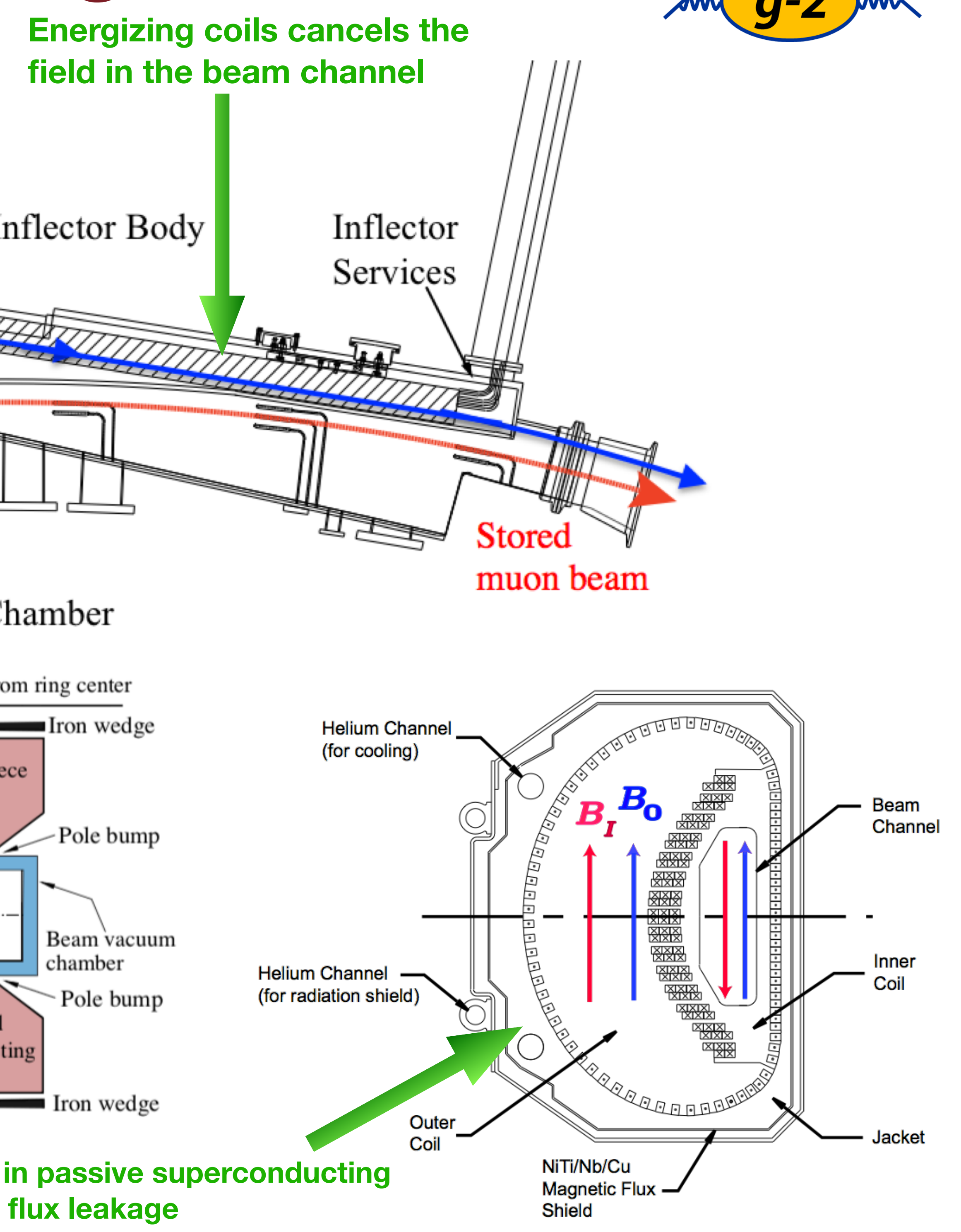

UMassAmherst

67 David Flay I Measuring the Muon Anomalous Magnetic Moment to High Precision 


\section{Theory Status of Hadronic Contribution to $a_{\mu}$}

honnomi<smiles>CC(C)(C)C(C)(C)C(C)(C)C</smiles>

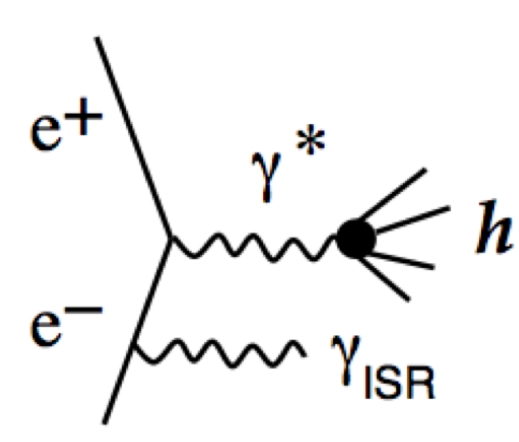

$$
\begin{aligned}
a_{\mu}^{\mathrm{had} ; \mathrm{LO}} & =\left(\frac{\alpha m_{\mu}}{3 \pi}\right)^{2} \int_{m_{\pi}^{2}}^{\infty} \frac{d s}{s^{2}} K(s) R(s) \\
R & \equiv \frac{\sigma_{\mathrm{tot}}\left(e^{+} e^{-} \rightarrow \text { hadrons }\right)}{\sigma\left(e^{+} e^{-} \rightarrow \mu^{+} \mu^{-}\right)}
\end{aligned}
$$

- Critical input to HVP from e+e-colliders (SND, CMD3, BaBar, KLOE, Belle, BESIII)

- BESIII: 3x more data available, luminosity measurement improvements

- VEPP-2000: Aiming for 0.3\% (fractional) uncertainty; radiative return + energy scan

- CMD3: Will measure up to 2 GeV (energy scan, ISR - good cross check)

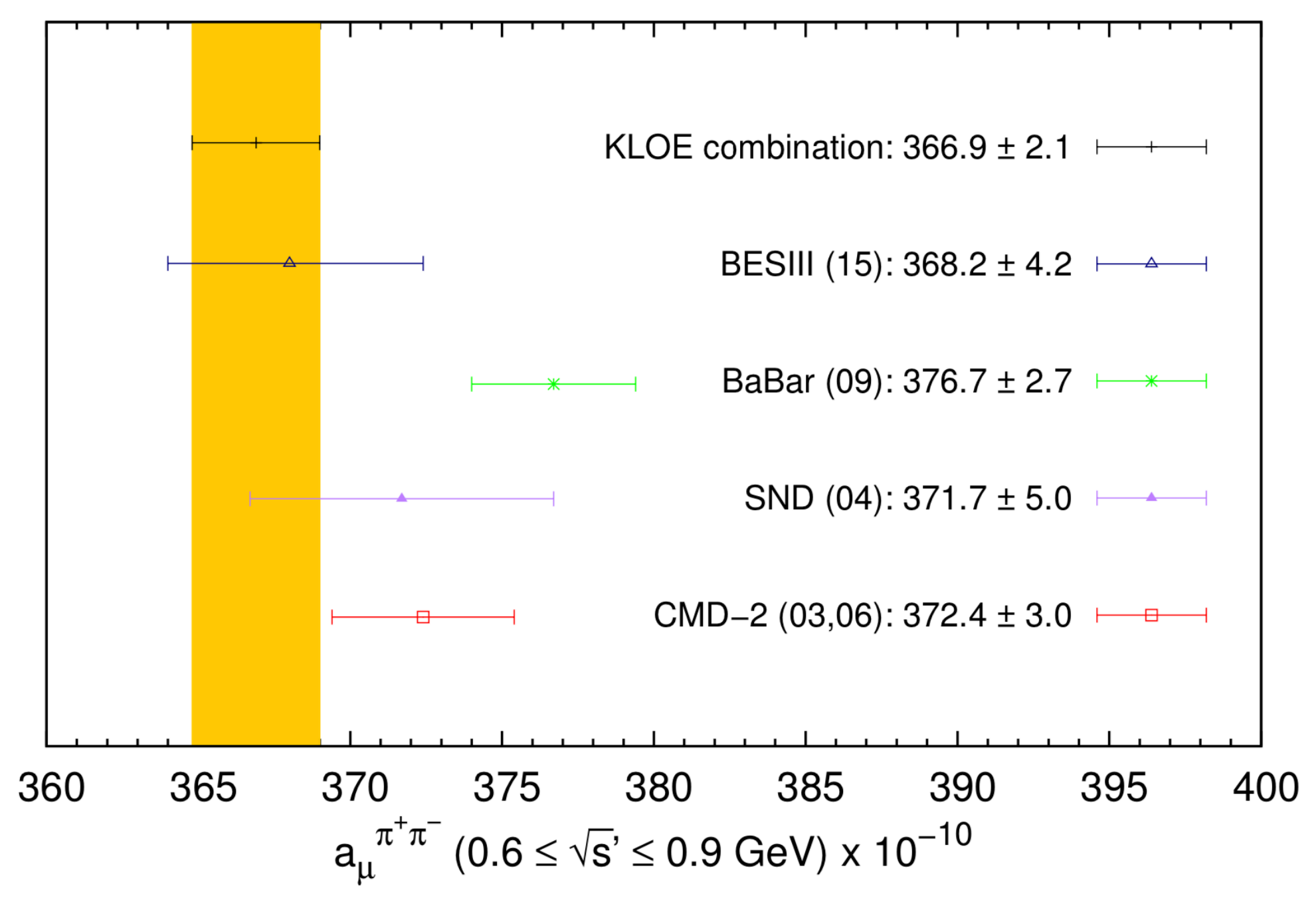

A. Anastasi et al., arXiv:1711.03085 [hep-ex]

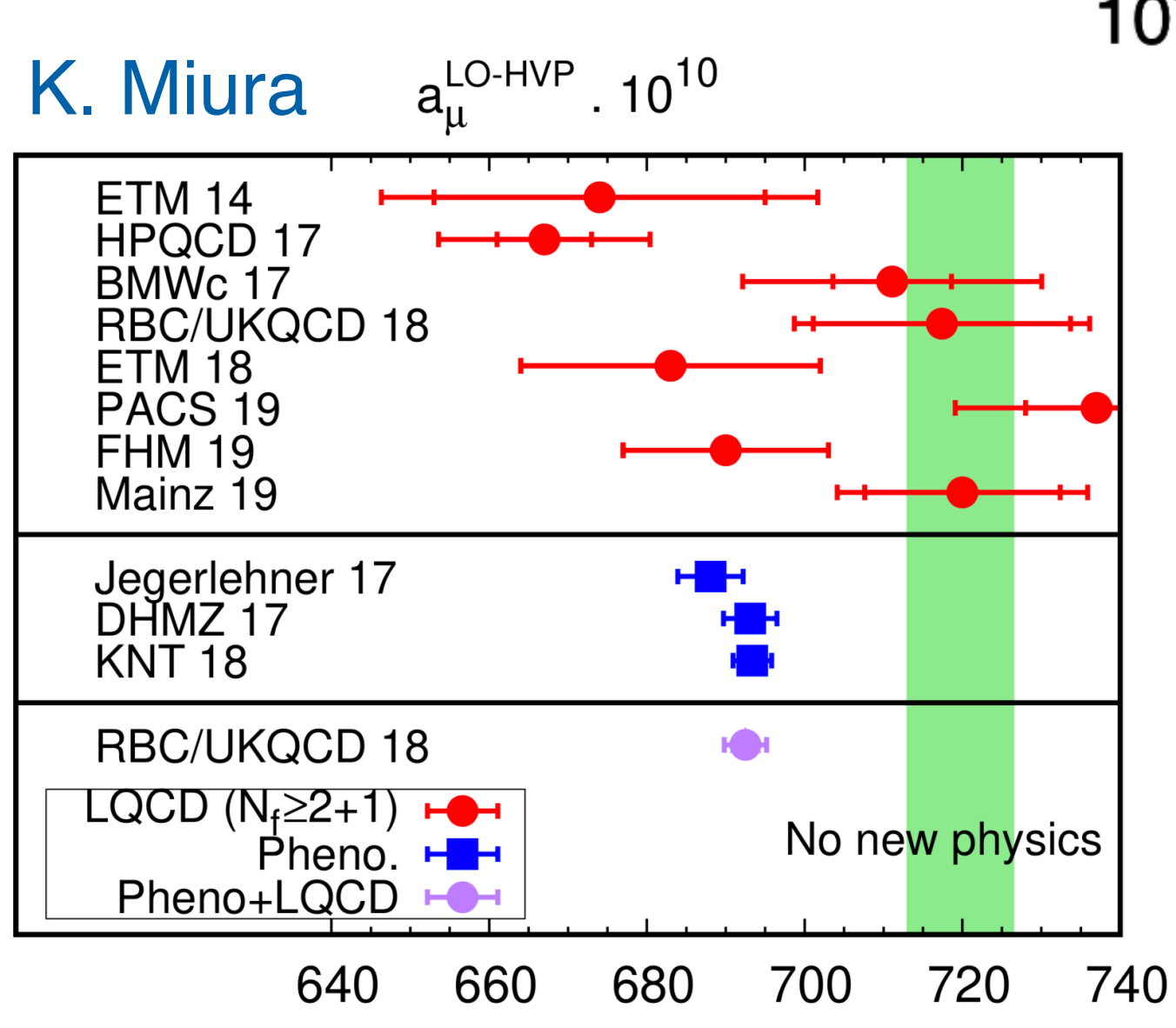

- Lattice calcullations of $a_{\mu} H V P$ to $1 \%$ soon, 30\% for HLbL in 3-5 years
M. Davier et al., arXiv:1706.09436 [hep-ph]

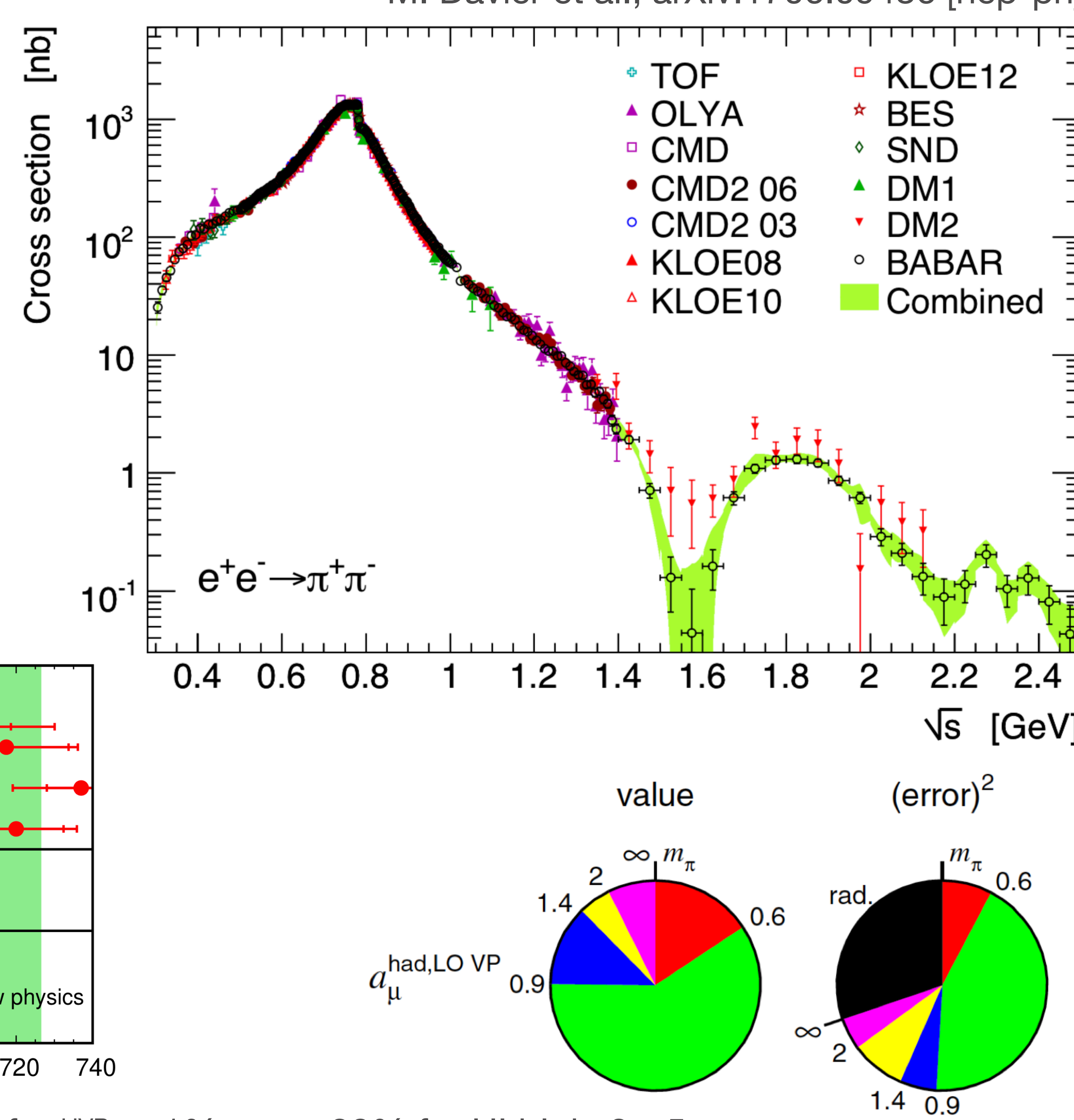

UMassAmherst 


\section{Physics Beyond the Standard Model?}

\section{SUSY, TeV-Scale Models}

- Higgs measured at the LHC to be $125 \mathrm{GeV}$

- Theory: Higgs should acquire much heavier mass from loops with heavy SM particles (e.g., top quark)

- Supersymmetry: new class of particles that enters such loops and cancels this contribution
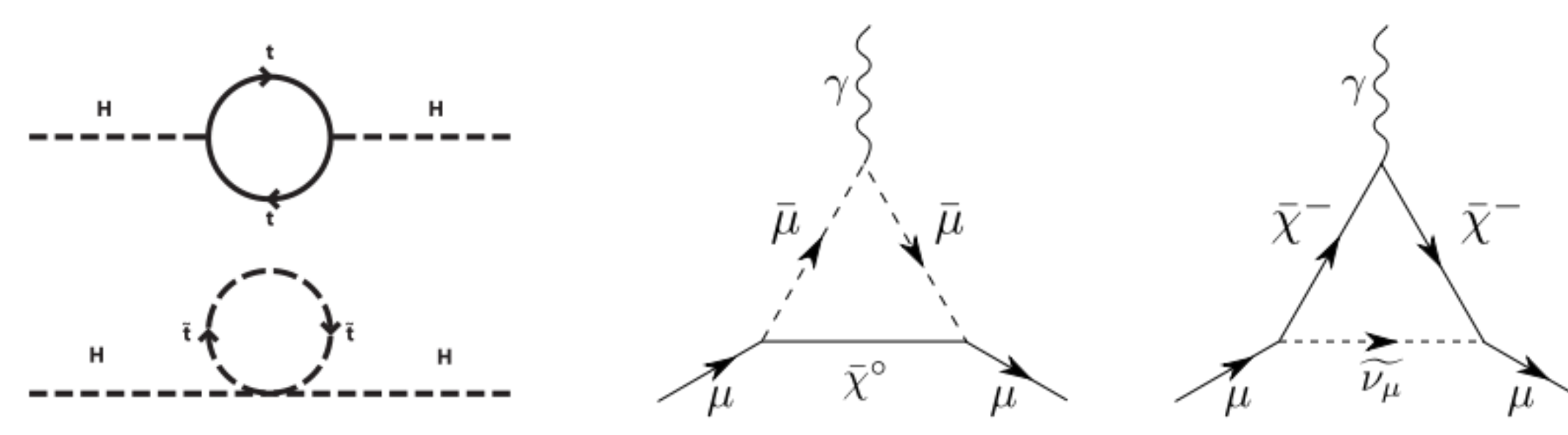

- Complementary to direct searches at the LHC

- Sensitivity to $\operatorname{sgn}(\mu), \tan (\beta)$

- Contributions to $a_{\mu}$ arise from charginos, sleptons

- LHC searches sensitive to squarks, gluinos

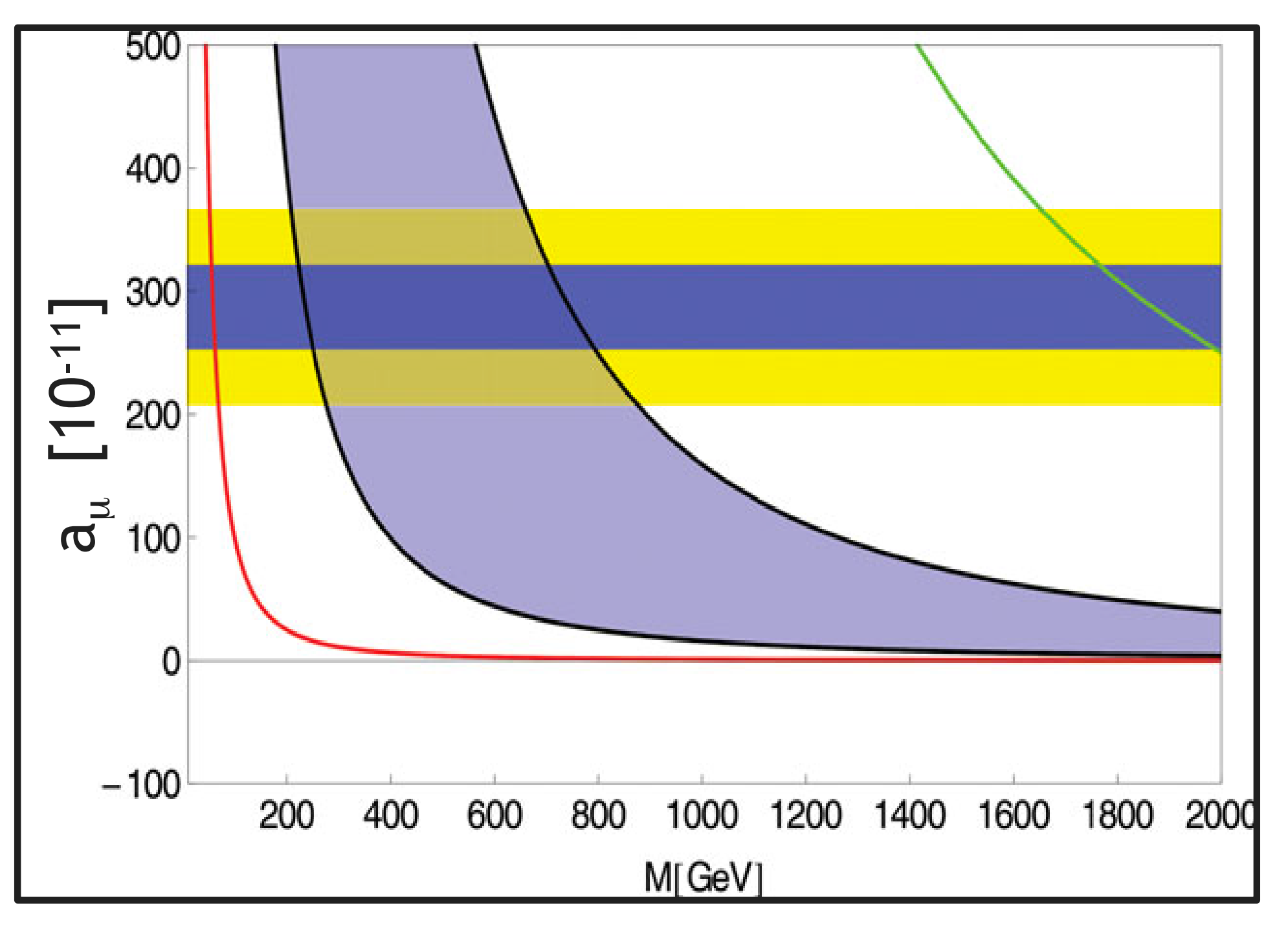

D. Hertzog, Ann. Phys. (Berlin), 2015, courtesy D. Stockinger

Z Z', W', UED, Littlest Higgs

- Assumes typical weak coupling

Radiative muon mass generation

Unparticles, Extra Dimension Models, SUSY ( $\tan \beta=5$ to 50)

\section{Z' Possibilities}
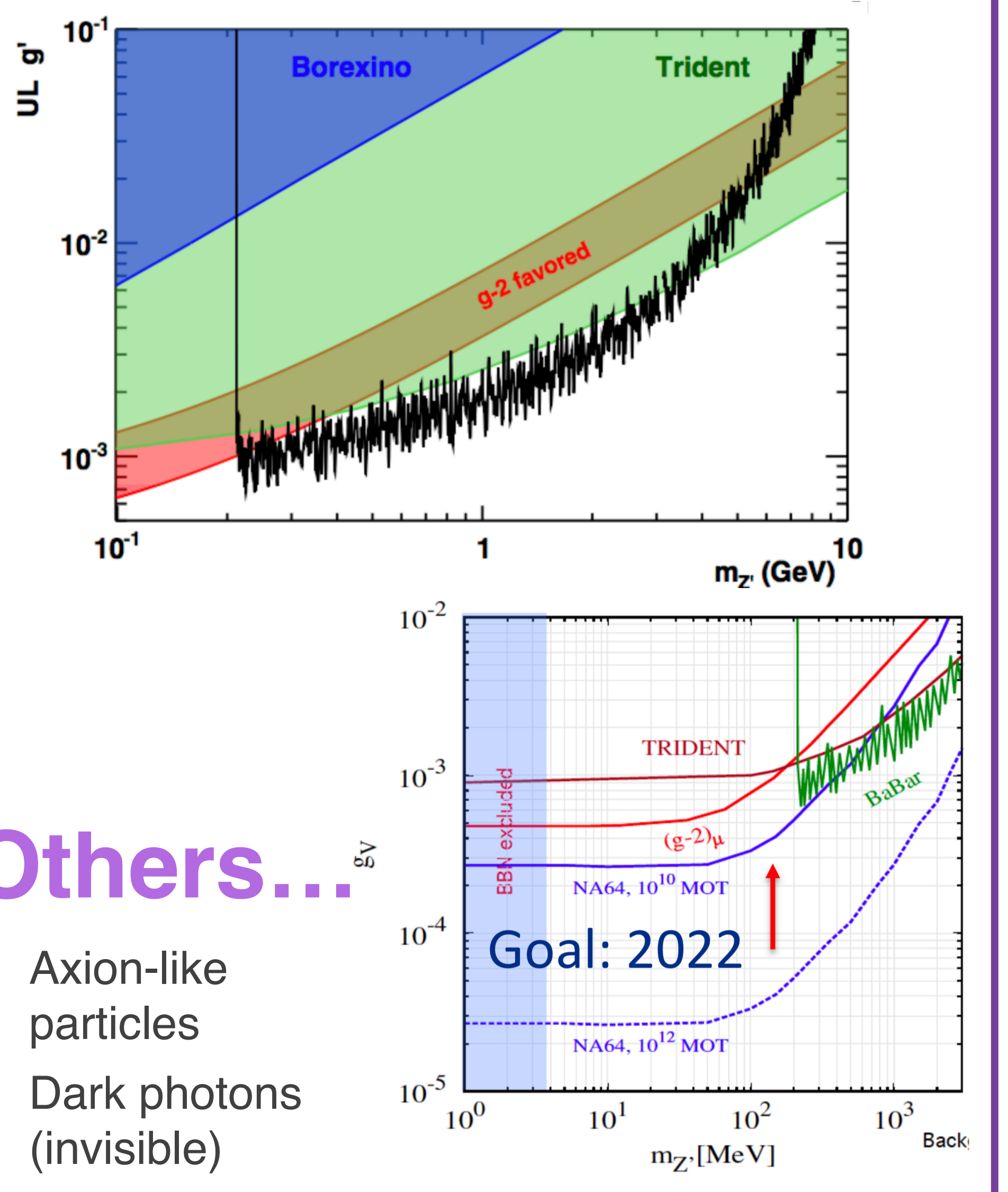

Extended Higgs/ leptoquarks 


\section{Analysis Details: $\omega_{a}$}

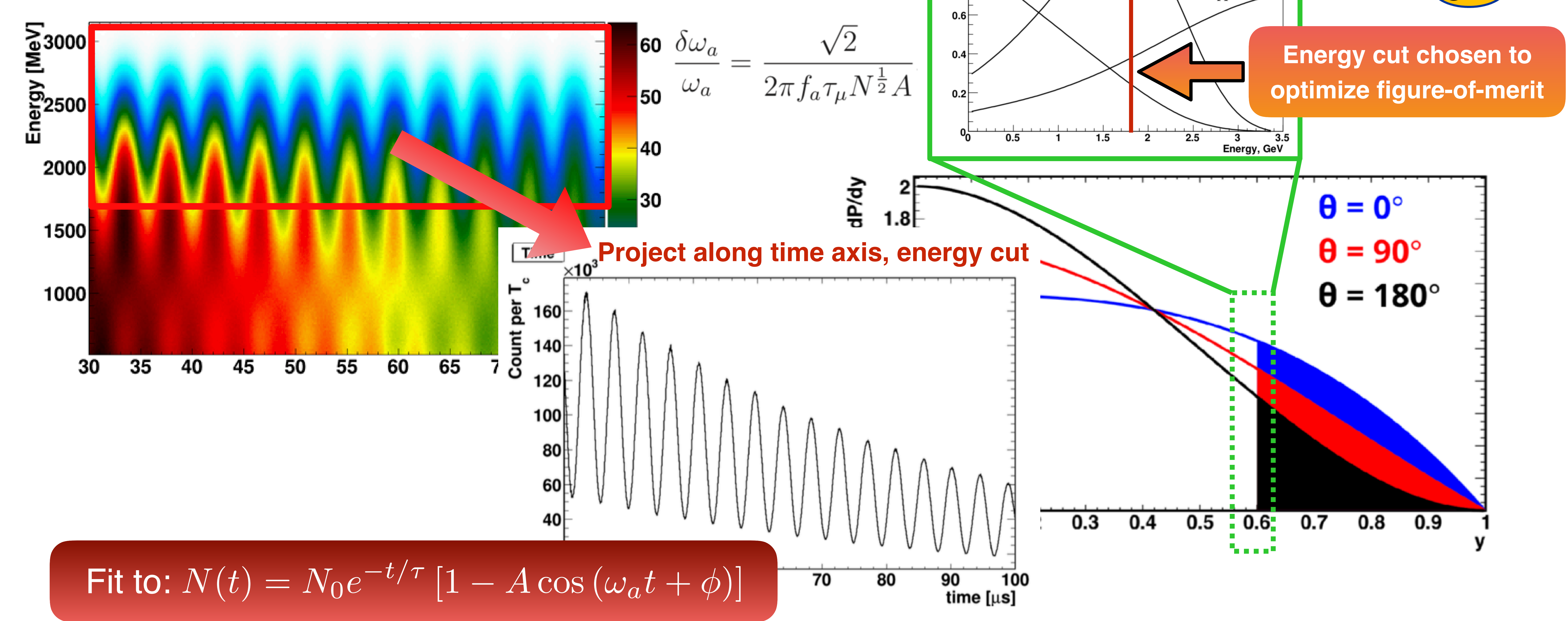




\section{Beam Dynamics Corrections}

- Full expression for $\omega_{a}$ :

$$
\begin{aligned}
& \vec{\omega}_{a}=\vec{\omega}_{S}-\vec{\omega}_{C}=-\frac{e}{m c}\left[a_{\mu} \vec{B}-\left(a_{\mu}-\frac{1}{\gamma^{2}-1}\right) \vec{\beta} \times \vec{E}-a_{\mu}\left(\frac{\gamma}{\gamma+1}\right)(\vec{\beta} \cdot \vec{B}) \vec{\beta}\right. \\
& \quad \text { Not all } \mu^{+} \text {at this } \mathrm{Y} \rightarrow \text { E-field correction }
\end{aligned}
$$

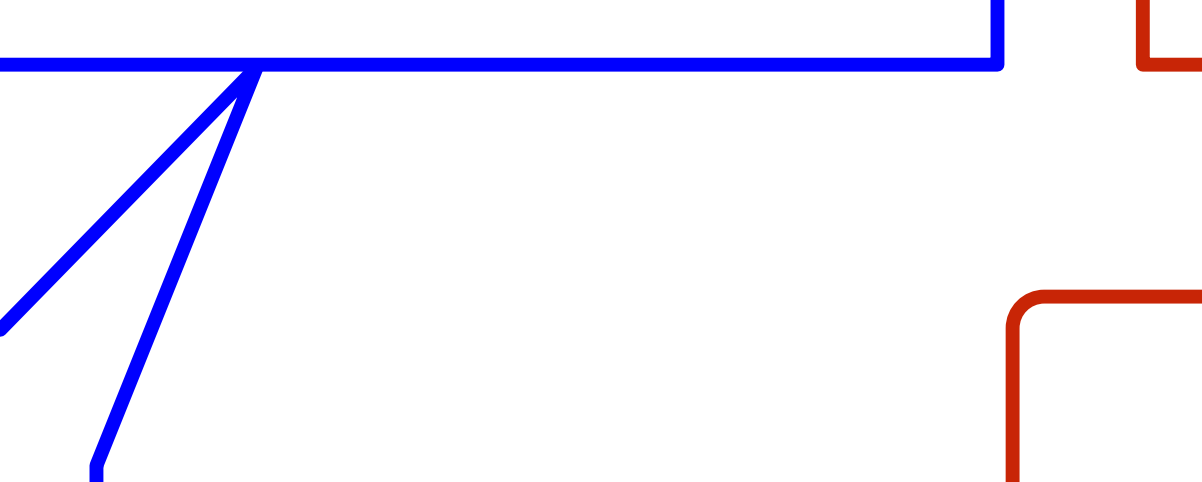

- Choose $Y=29.3\left(p_{\mu}=3.094 \mathrm{GeV} / \mathrm{c}\right)$
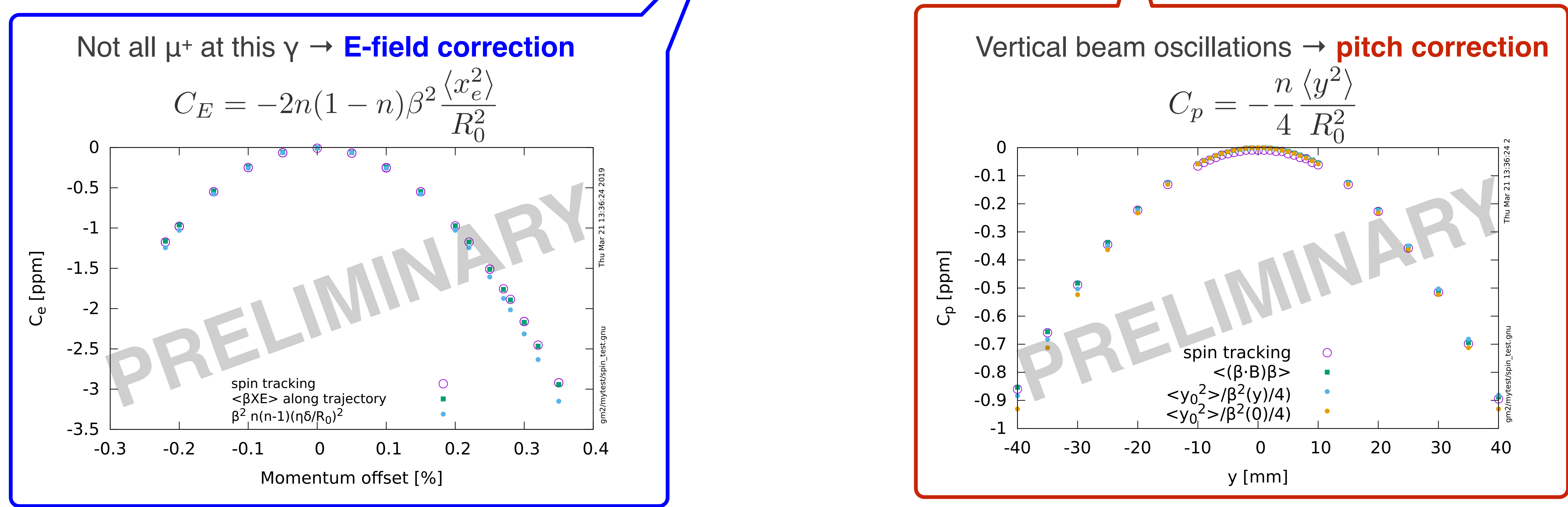


\section{How is a Kick Made?}

- A charging power supply charges up a capacitor bank to $700 \mathrm{~V}$

- Capacitors are discharged through a transformer into a Blumlein (a HV capacitor up to $55 \mathrm{kV}$ )

- Current in Blumlein is discharged into a resistive load $(Z=12.5 \Omega)$

- Current delivered to plates, producing a $200 \mathrm{G}$ magnetic field, rotating muon's momentum vector

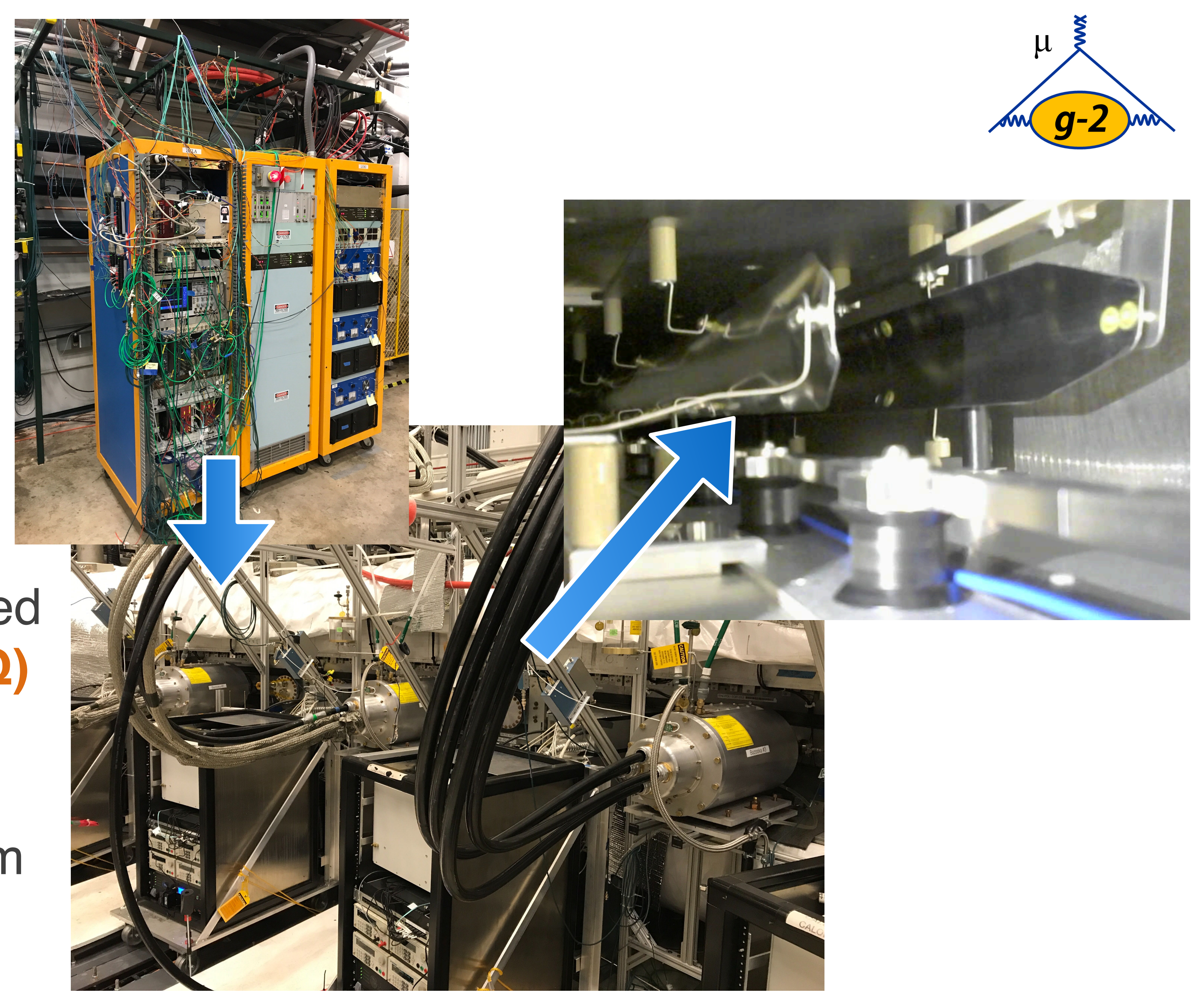




\section{What Affects the Beam Shape?}

- Kicker pulse strength, shape affects structure of beam

- Beam width affected by dynamics

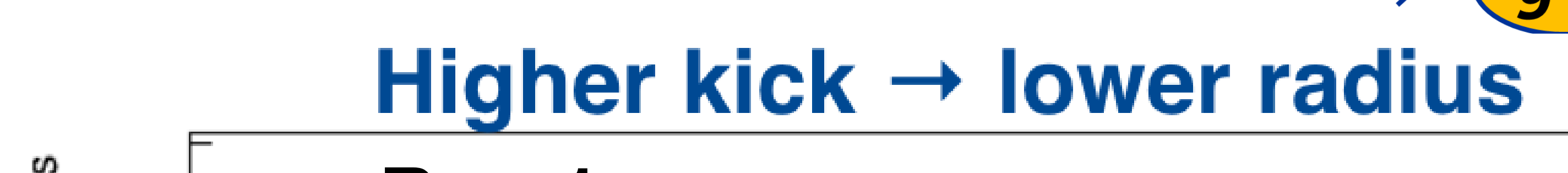

Higher kick $\rightarrow$ lower radius
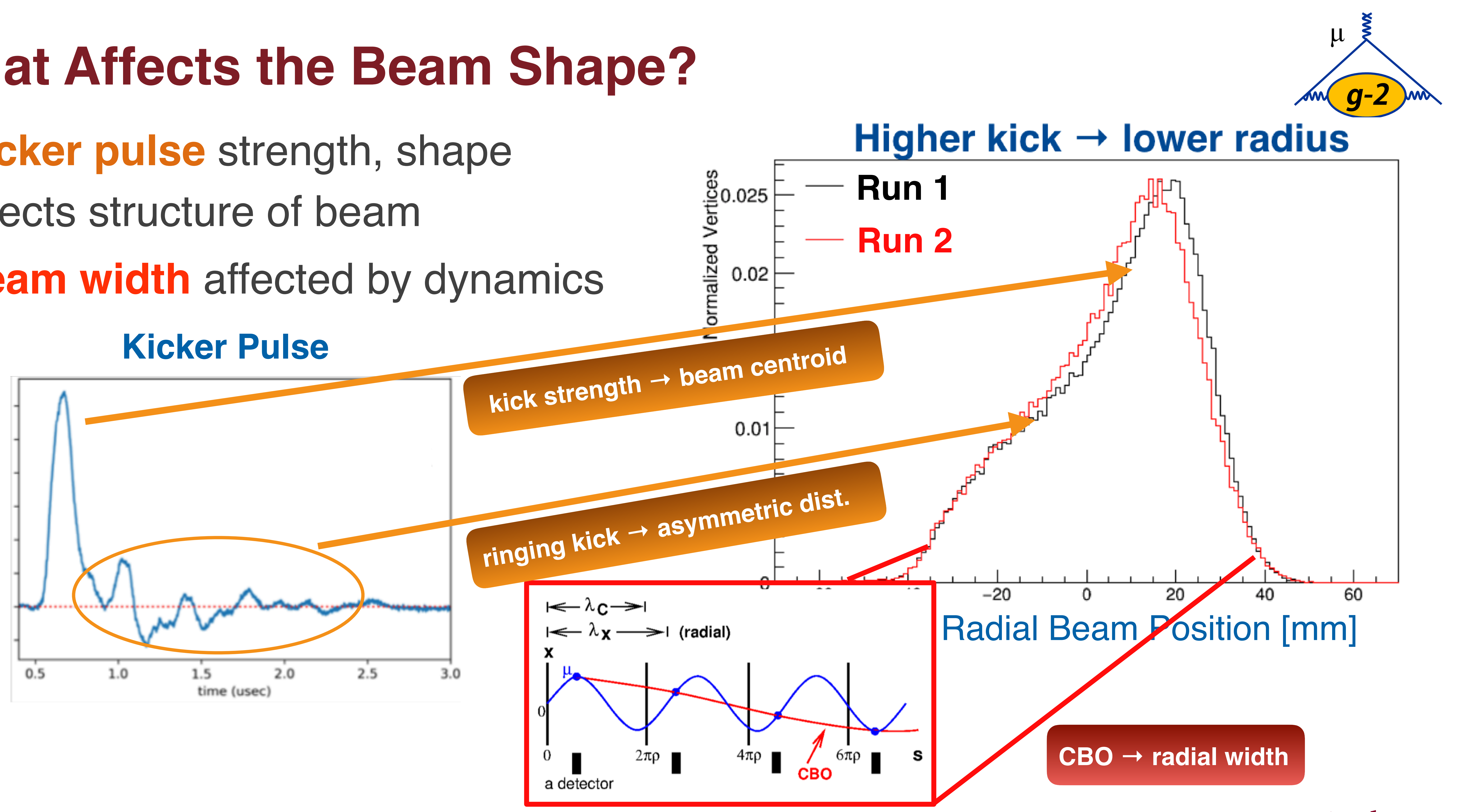

UMassAmherst

76 David Flay I Measuring the Muon Anomalous Magnetic Moment to High Precision 


\section{Pulsed Nuclear Magnetic Resonance}

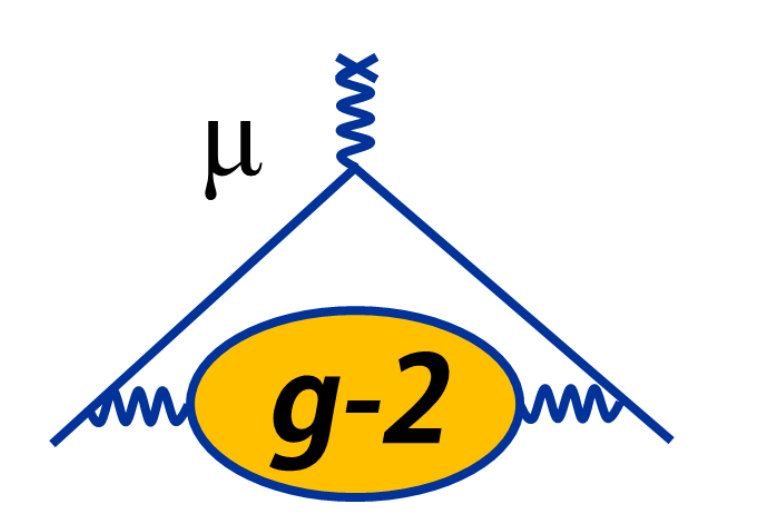

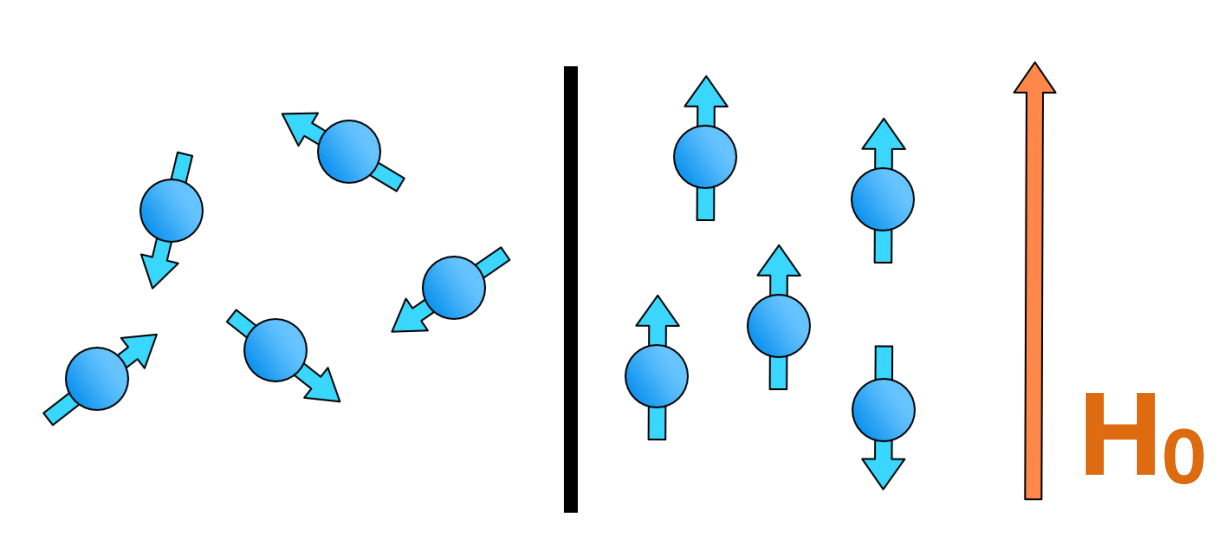

$B=0$

$B \neq 0$
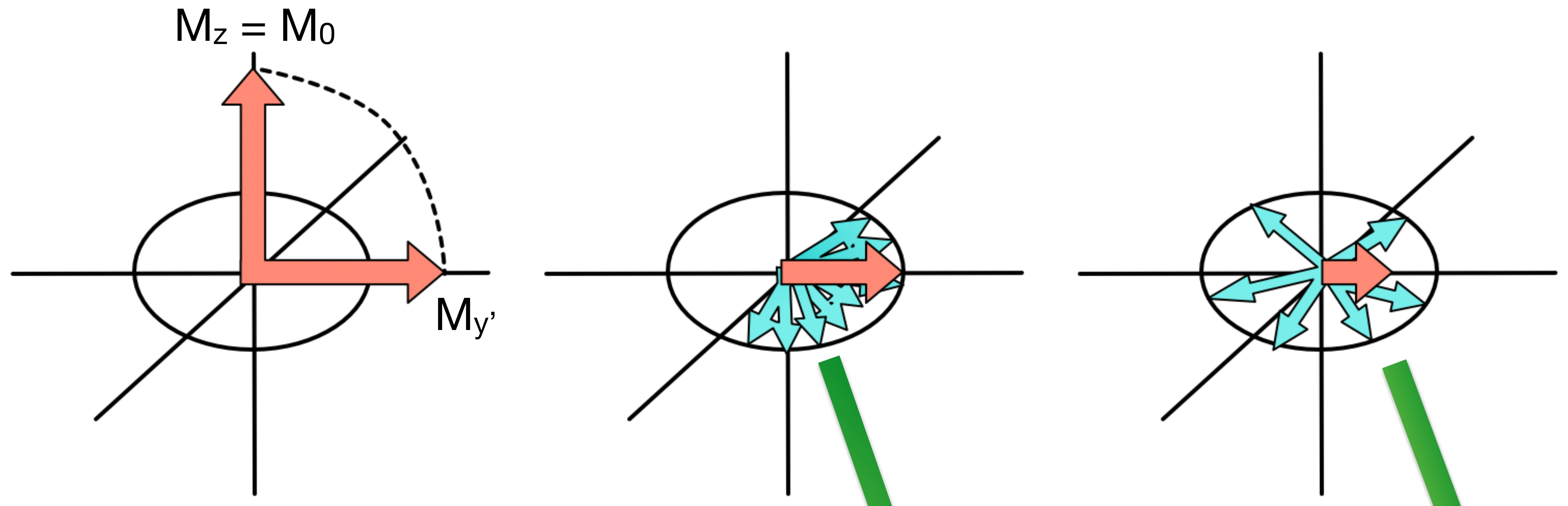

- Apply an RF pulse for a short time to the sample at Larmor frequency - tips spins perpendicular to external B field ( $\pi / 2$ pulse)

- Spin precession induces an EMF in the pickup coil

- So-called Free-Induction Decay (FID)

- Decay of signal driven by:

- Spin-spin interactions (dephasing) (pure $T_{2}$ )

- Field inhomogeneities $\left(\mathrm{T}_{2}{ }^{*}\right)$

- Simultaneously, spins relax back to alignment with holding field (spin-lattice relaxation, $\mathrm{T}_{1}$ )

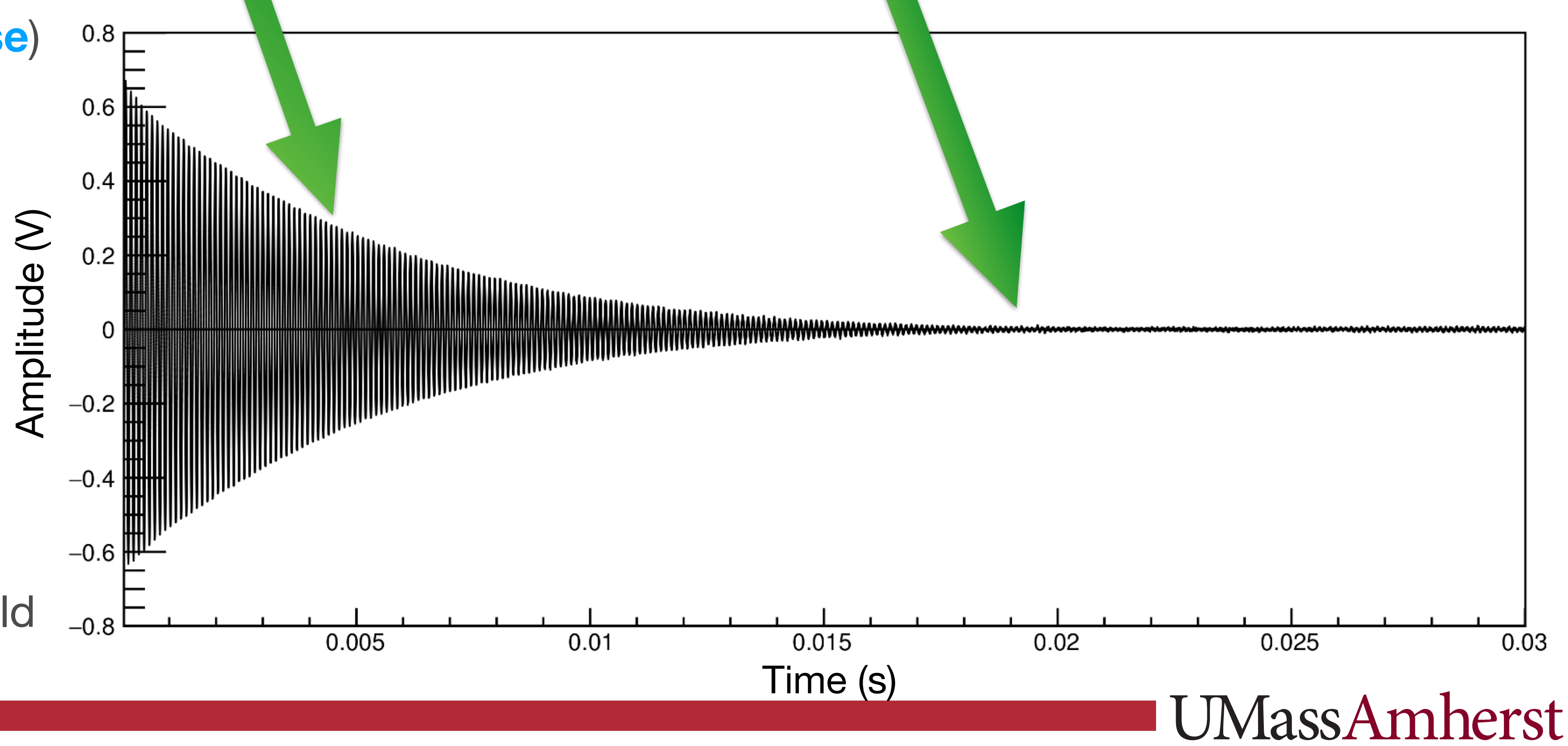

79 David Flay I Measuring the Muon Anomalous Magnetic Moment to High Precision 


\section{Magnetic Circuits}

$$
\begin{aligned}
& \mathcal{E}=\oint \overrightarrow{f_{s}} \cdot d \vec{\ell}=V=I R \quad \begin{array}{l}
\text { Can write a similar } \\
\text { equation for magnets }
\end{array} \\
& \mathcal{F}=\oint \vec{H} \cdot d \vec{\ell}=N I \quad \begin{array}{l}
\text { Magnetomotive Force (mmf) } \\
\vec{B}=\mu_{0}\left(1+\chi_{m}\right) \vec{H}=\mu \vec{H}
\end{array} \\
& \Phi=\vec{B} \cdot \vec{A}=\mu \vec{H} \cdot \vec{A} \quad \text { Consider } \mathbf{H} \text { in terms of B } \\
& \Phi \oint \frac{d \ell}{\mu A}=\mathcal{F} \Rightarrow \mathcal{R}=\oint \frac{d \ell}{\mu A}=\frac{\mathcal{F}}{\Phi}
\end{aligned}
$$

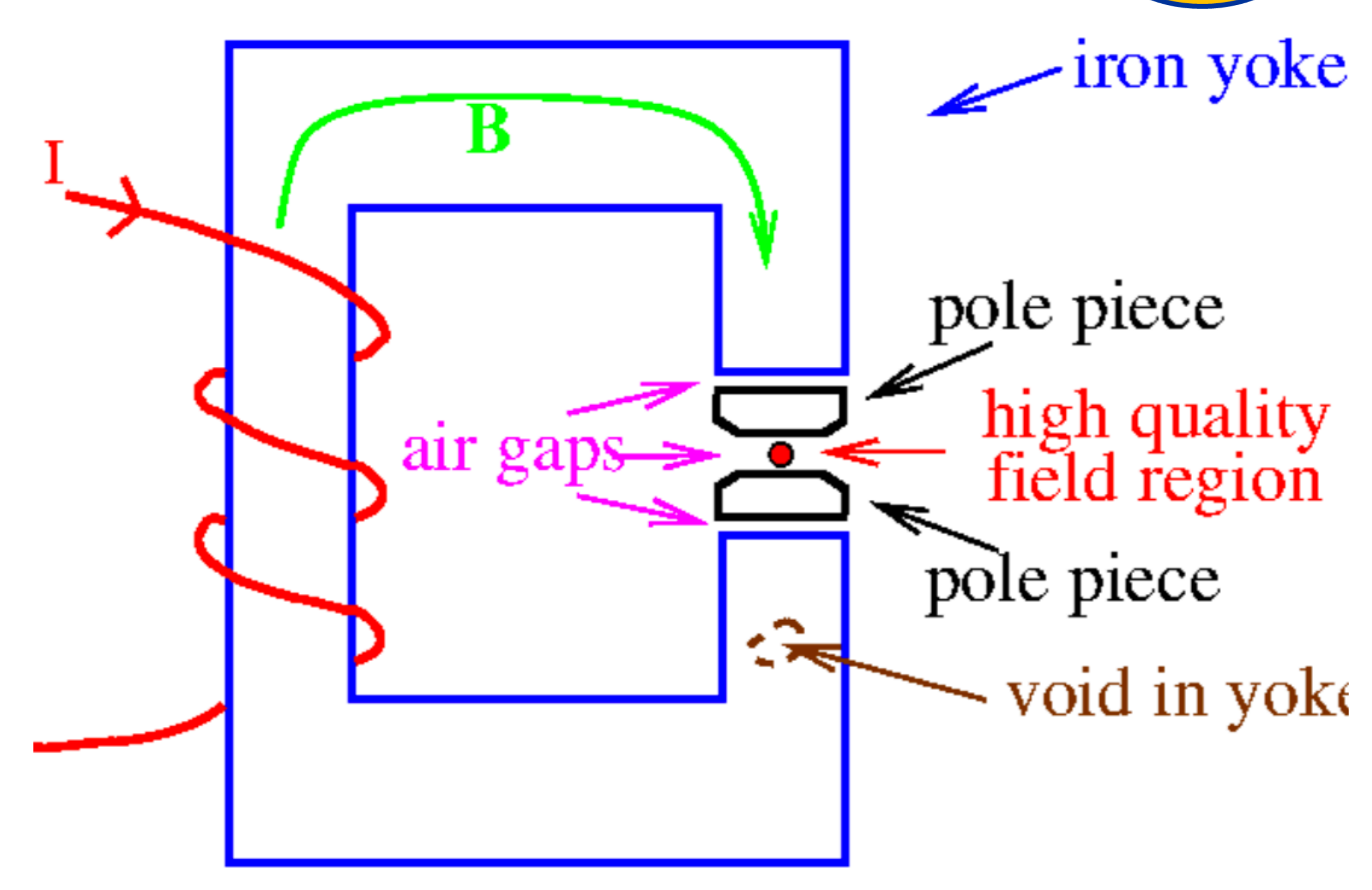

\section{Magnetic Reluctance}

- Analogous to resistance in an electrical circuit

$$
V=I R \Leftrightarrow \mathcal{F}=\Phi \mathcal{R}
$$

- Current flows along a path of least resistance while field lines will take a path of least reluctance

- While the emf drives electric charges (Ohm's Law), the mmf "drives" magnetic field lines (Hopkinson's Law) 


\section{Magnet Anatomy}

- For E821, Gordon Danby had a brilliant magnet design

$B=1.45 \mathrm{~T}(\sim 5200 \mathrm{~A})$

- Non-persistent current: fine-tuning of field in real time

12 C-shaped yokes

- 3 upper and 3 lower poles per yoke

- 72 total poles

\section{Shimming knobs}

- Pole separation determines field: pole tilts, non-flatness affect uniformity

- Top hats (30 deg effect, dipole)

- Wedges (10 deg effect, dipole, quadrupole)

- Edge shims (10 deg effect, dipole, quadrupole, sextupole)

- Laminations (1 deg effect, dipole, quadrupole, sextupole)

- Surface coils (360 deg effect, quadrupole, sextupole,...)
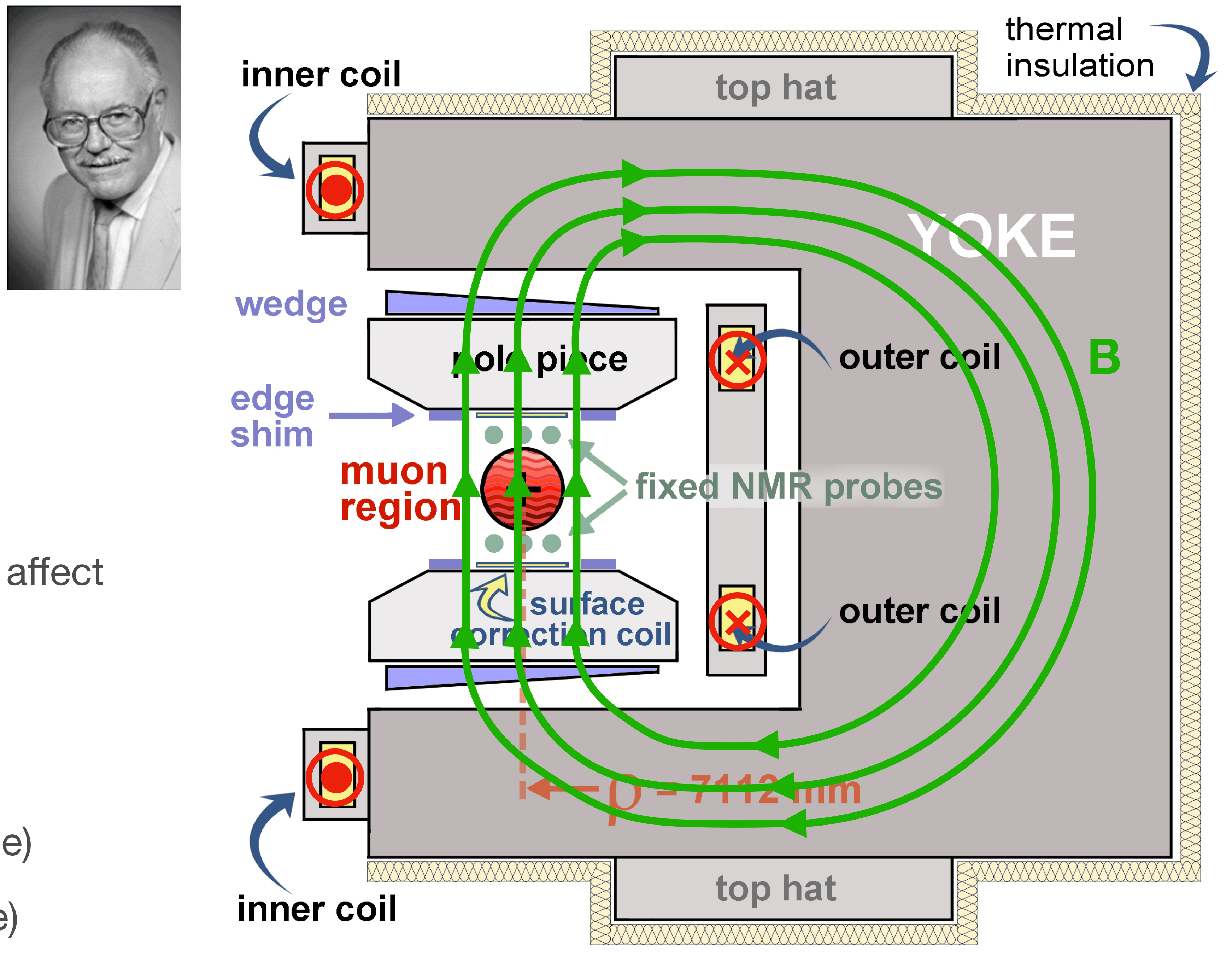

Current direction indicated by red markers

81 David Flay I Measuring the Muon Anomalous Magnetic Moment to High Precision 


\section{Optimizing the Dipole Moment}

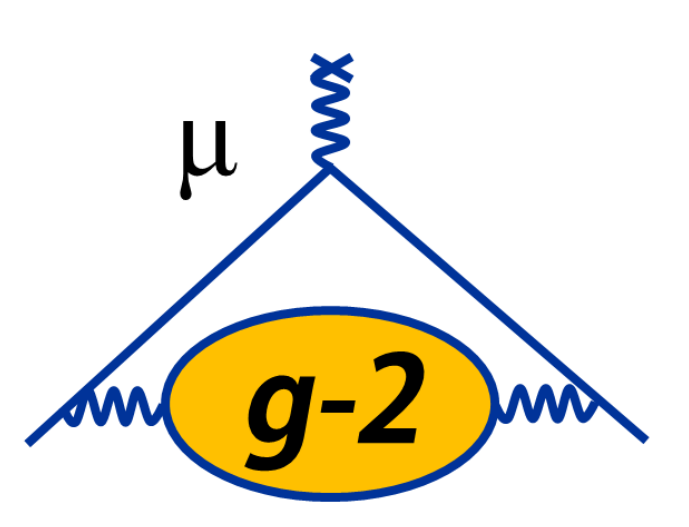

- Want to optimize the vertical component of the field

- Step and tilt discontinuities in pole surfaces yield large variations in the field

- To reduce/remove such effects, make adjustments to pole feet, which changes the magnet gaps and tilts

- Use 0.001-0.010" thick shims

- Requires removal of poles from the ring

- Informed by a computer model that optimizes the pole configurations

- Requires global continuity between pole surfaces

- Allows only three adjacent poles to be moved at a time (preserves alignment)

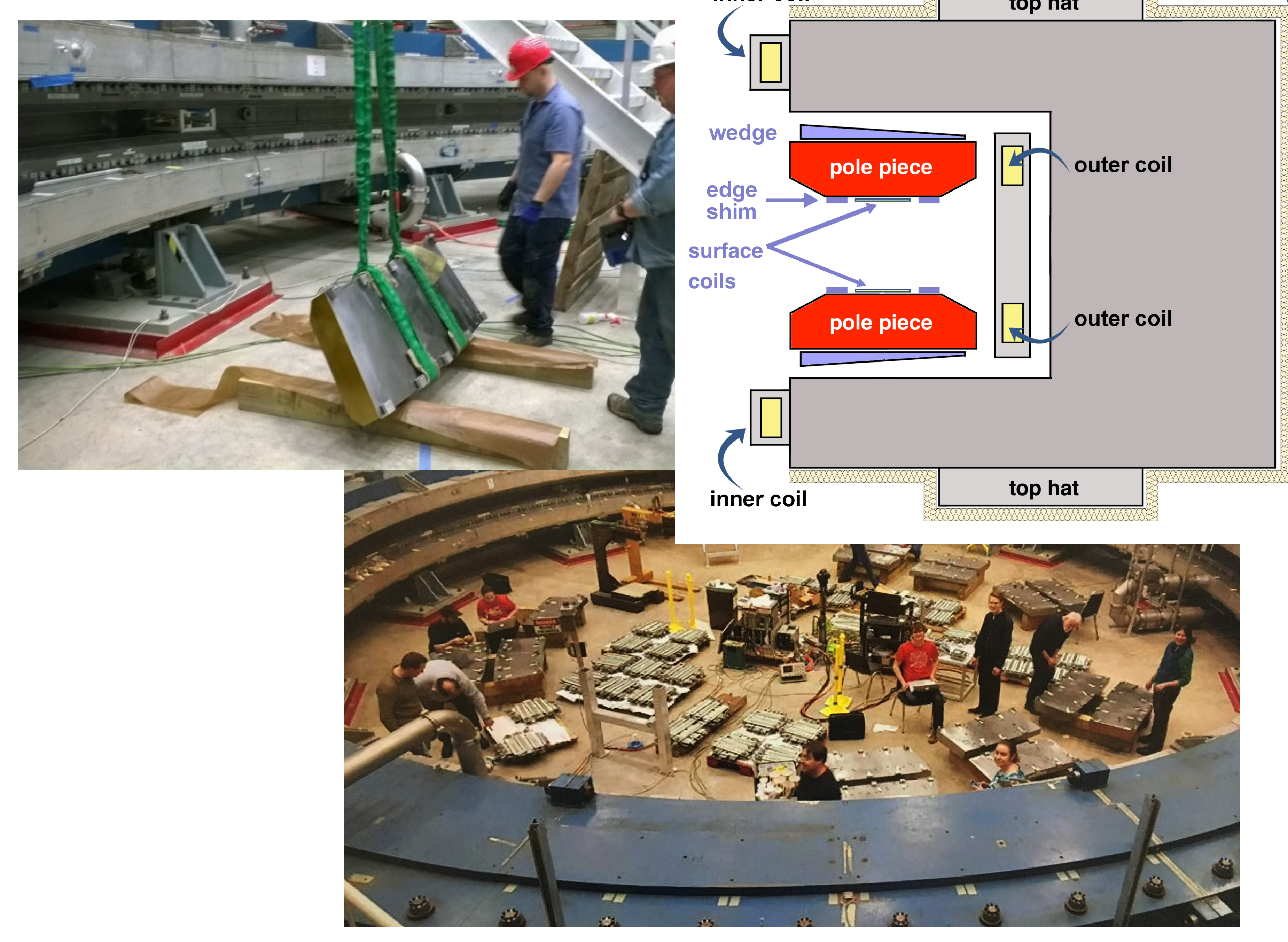




\section{Minimizing the Quad, Sext, Octu Moments}

Calibrated shimming knobs

- 48 top hats

- 864 wedges

- 8400 iron foils (on pole surfaces)

Coarse tuning: top hat \& wedge adjustments (dipole, quadrupole)

- Least-squares fit to field maps predicts top hat and wedge positions

Fine tuning: iron foils (quadrupole, sextupole,....)

- Modeled as saturated dipoles in 1.45 T field

- Computer code predicts foil width (mass) distribution to fill in the valleys of the field map
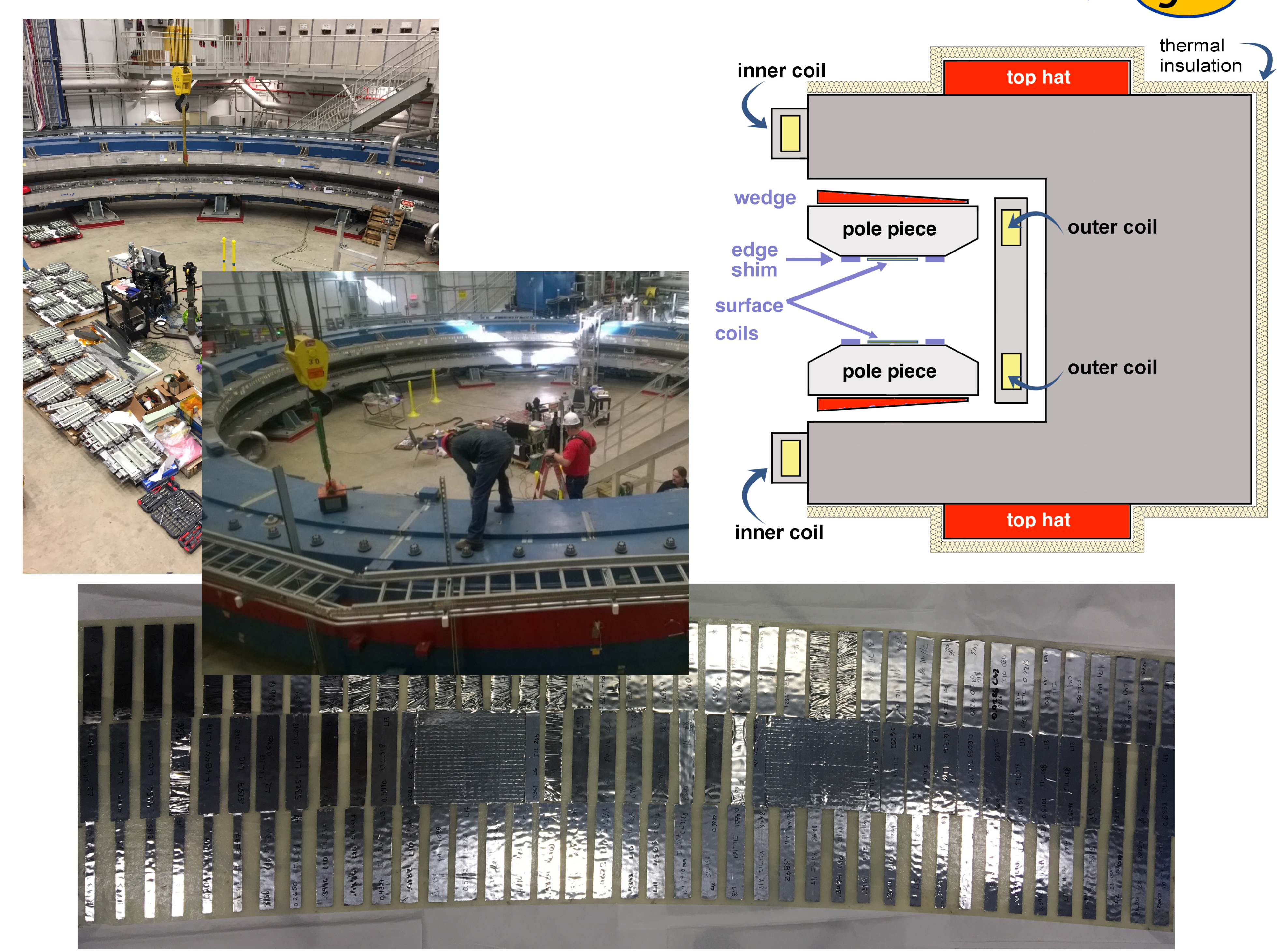


\section{Rough Shimming Results}
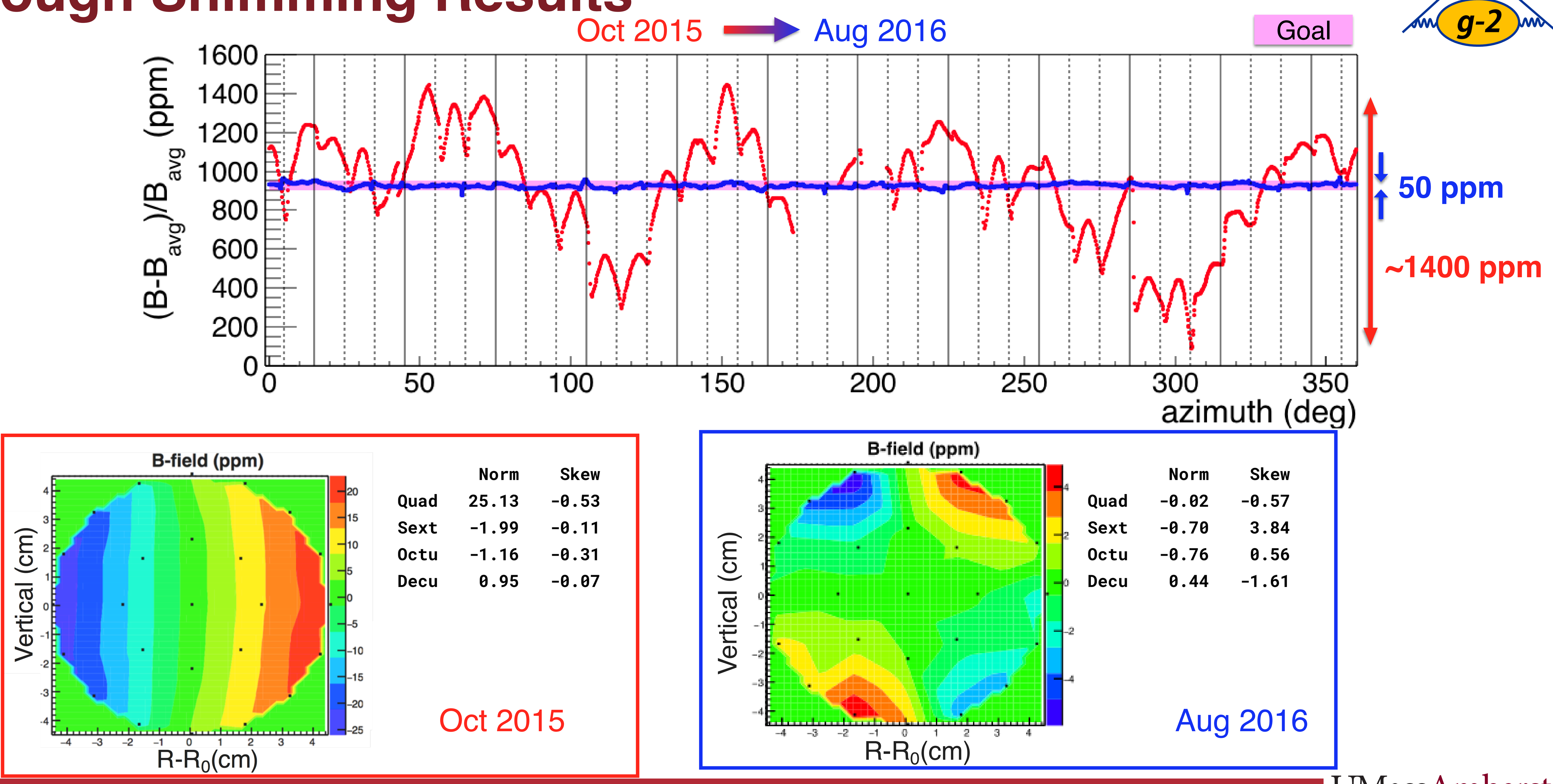

$\begin{array}{rr}\text { Norm } & \text { Skew } \\ -0.02 & -0.57 \\ -0.70 & 3.84 \\ -0.76 & 0.56 \\ 0.44 & -1.61\end{array}$

Aug 2016 


\section{Magnetic Field Comparison: BNL 821 and FNAL E989}

Dipole Vs Azimuth

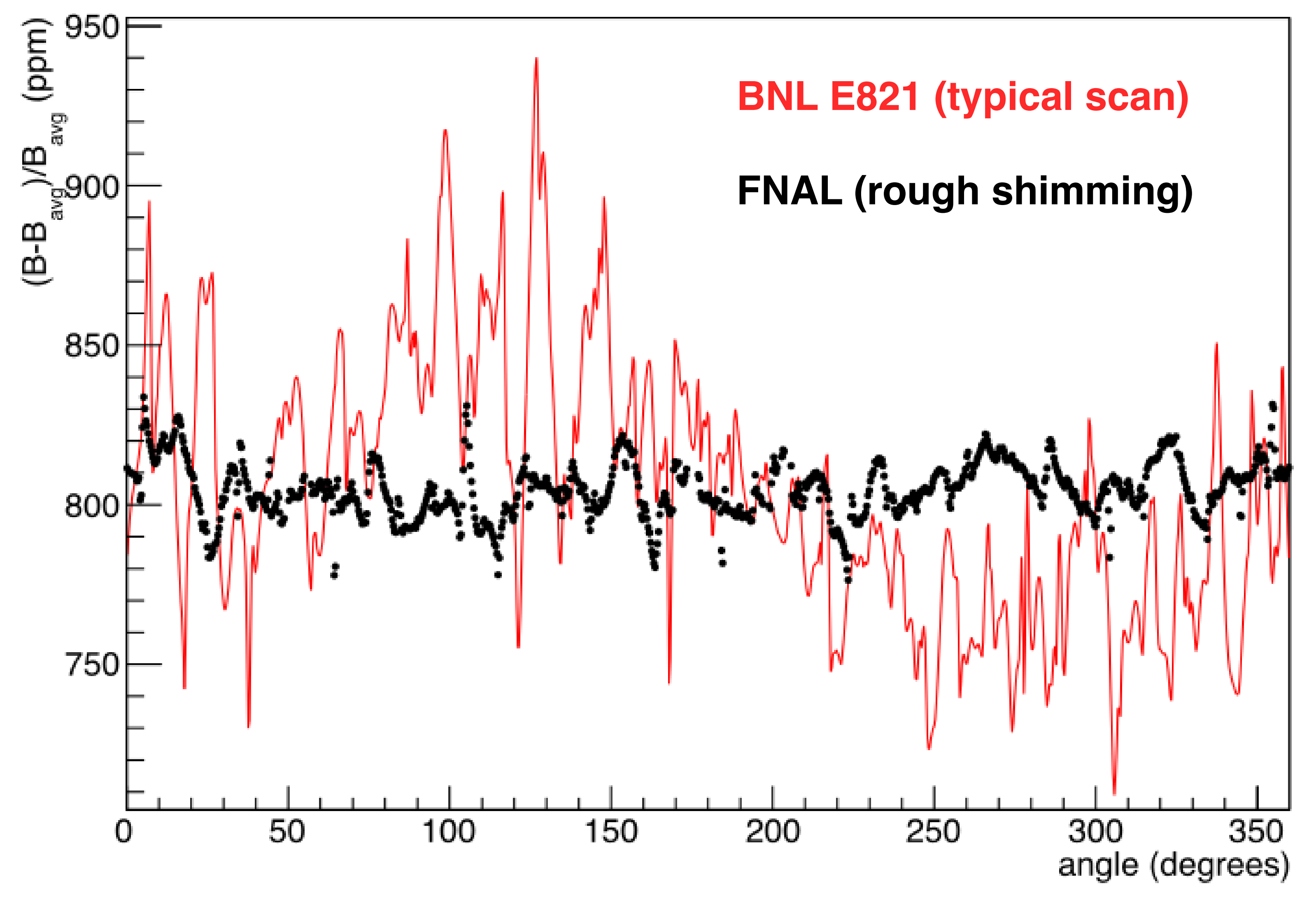

- Laminations very successful in reducing field variations

- BNL E821: 39 ppm RMS (dipole), 230 ppm peak-to-peak

- FNAL rough shimming: 10 ppm RMS (dipole), 75 ppm peak-to-peak 


\section{Magnetic Field Variations}

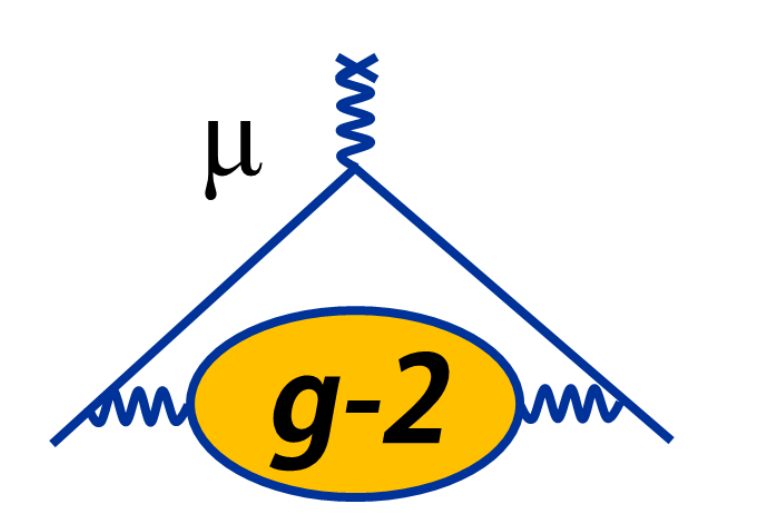

First Magnetic Field Map, Oct 142015

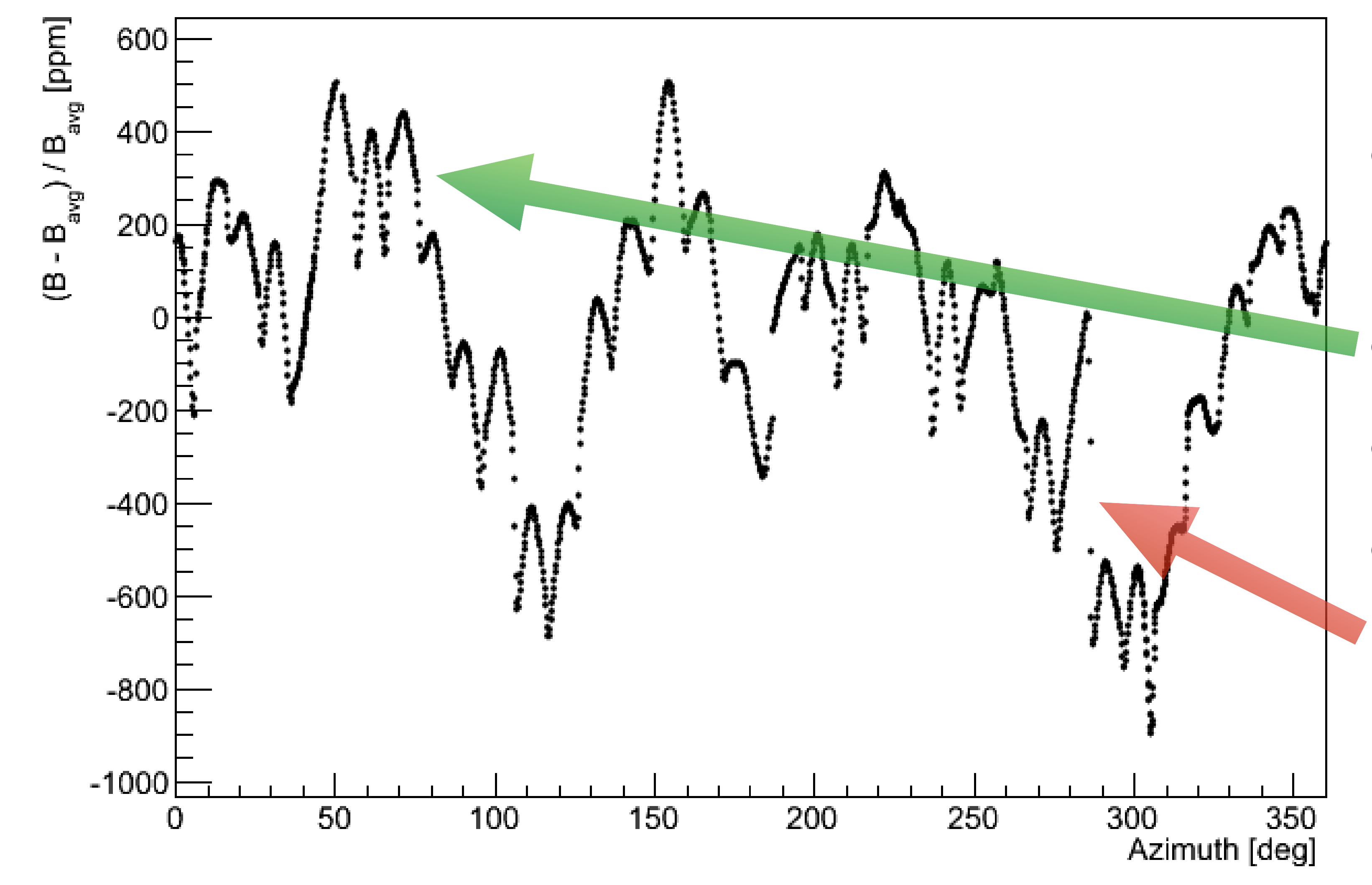

- Gradual drift from materials, pole gap changes

- 36 pairs of poles $\rightarrow 10$-degree structure

- Pole shape:

- Pole-to-pole discontinuities 


\section{Auxiliary Field Systems}

\section{Surface Correction Coils}

- Continuous PCB traces going around the ring on pole surfaces

- 100 concentric traces on upper poles, 100 on lower poles

- Programmable range: \pm 20 ppm on the field

- Used to cancel higher-order multipole moments in the magnetic field (on average)
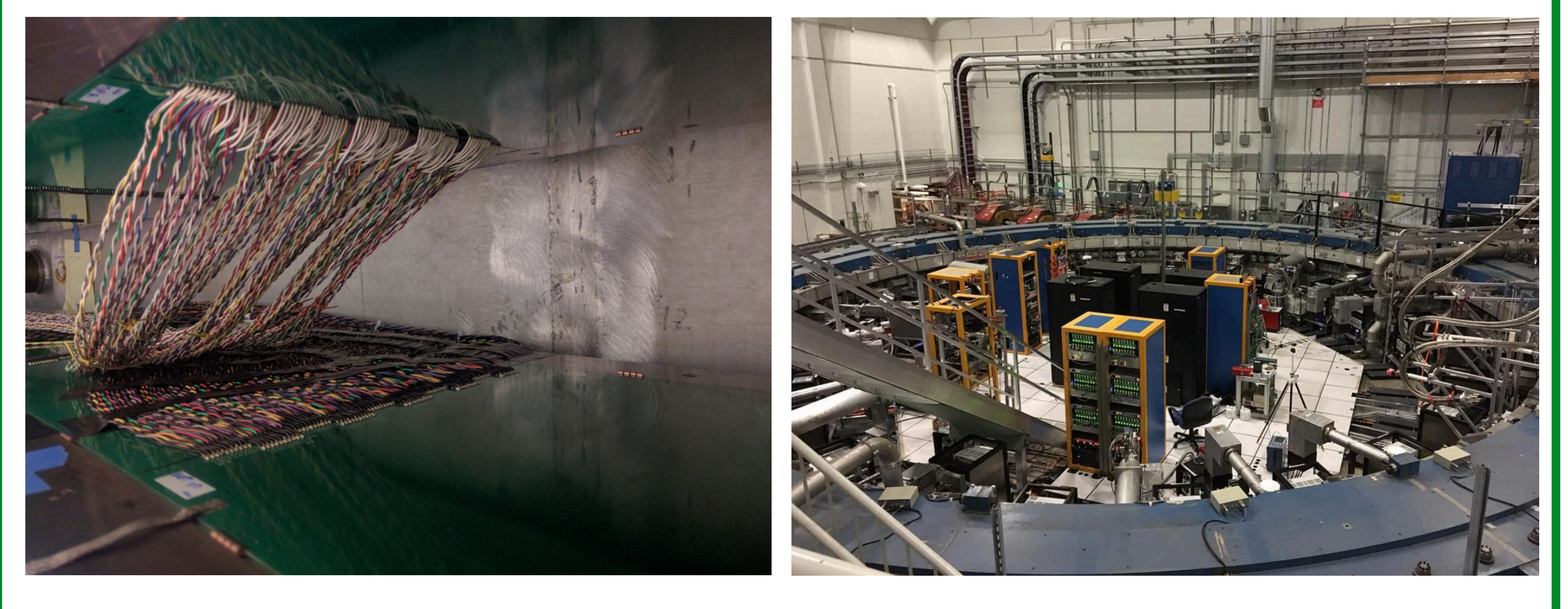

\section{Power Supply Feedback}

- Programmable current source with a range of $\pm 5 \mathrm{ppm}$ on the field

- Uses data from fixed probe system to stabilize the field at a specified set point

\section{Fluxgates}

- Measure $(x, y, z)$ components of transient fields in the hall

- Sensitive down to 10-9 T (DC or AC) fields

- Bandwidth up to $1 \mathrm{kHz}$

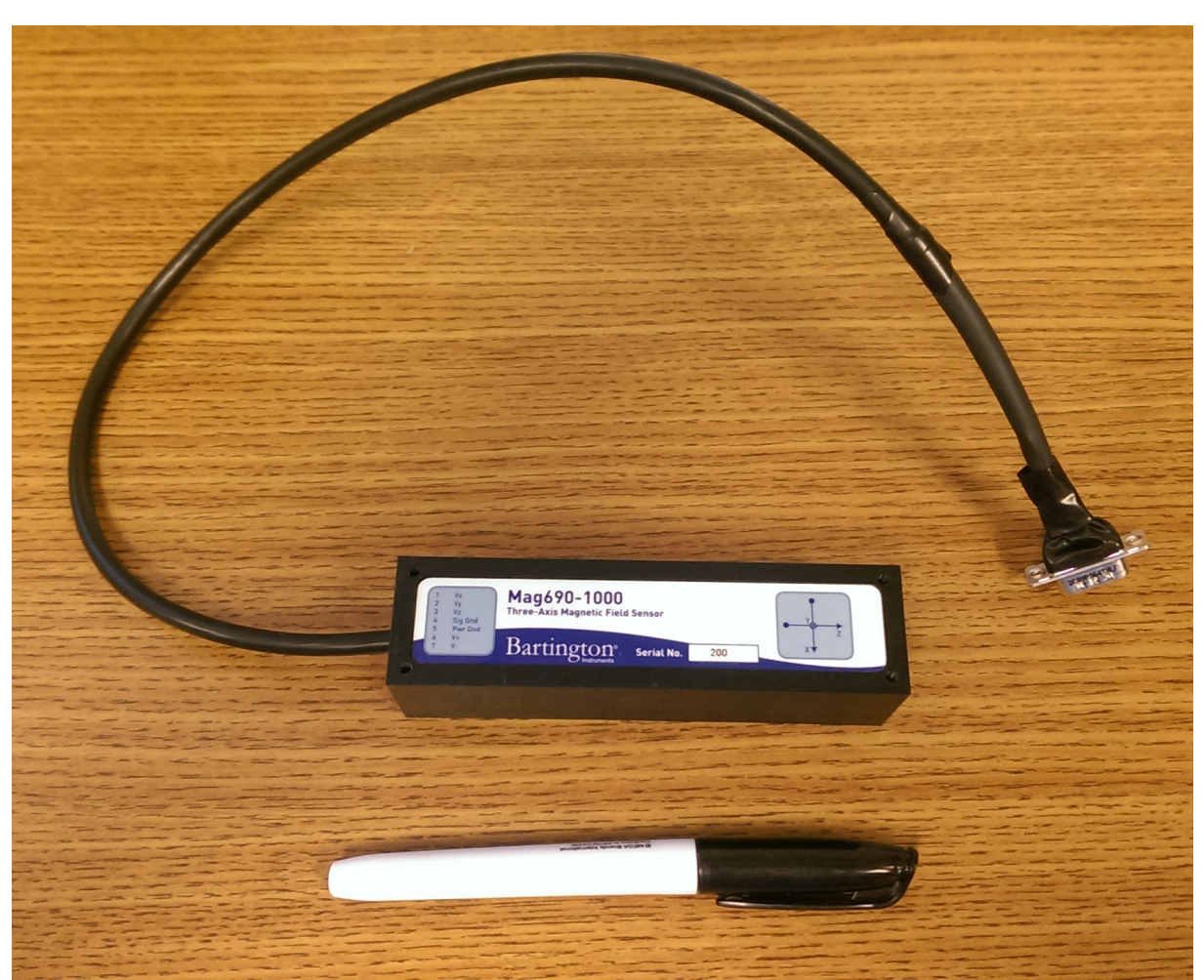




\section{Magnet Insulation}

- Temperature variations in the hall affect the quality of the magnetic field

- Observed $\sim 20 \mathrm{ppm} / \mathrm{deg} \mathrm{C}$ effects on the dipole moment during the run

- Also affects ability to track higher-order multipoles

- Two main issues

- Large changes in average temperature over time $\left(2-3^{\circ} \mathrm{C}\right)$

- Differential changes across the magnet $\left(\sim 3^{\circ} \mathrm{C}\right)$

- Two-pronged solution:

- Improved cooling system in the hall

- Install fiberglass insulation blanket on magnet steel

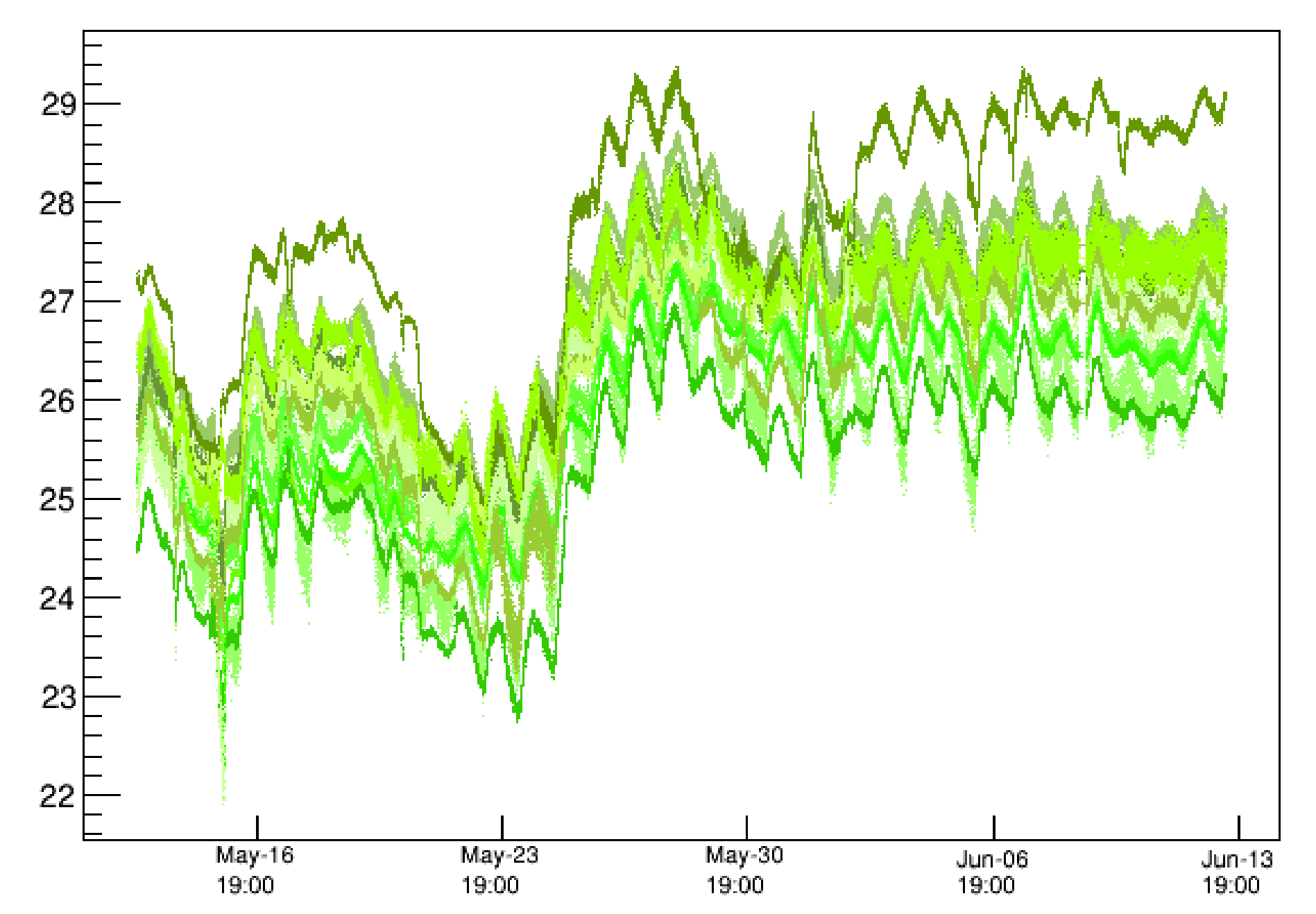

Fixed probe on yoke D vacuum chamber (top)
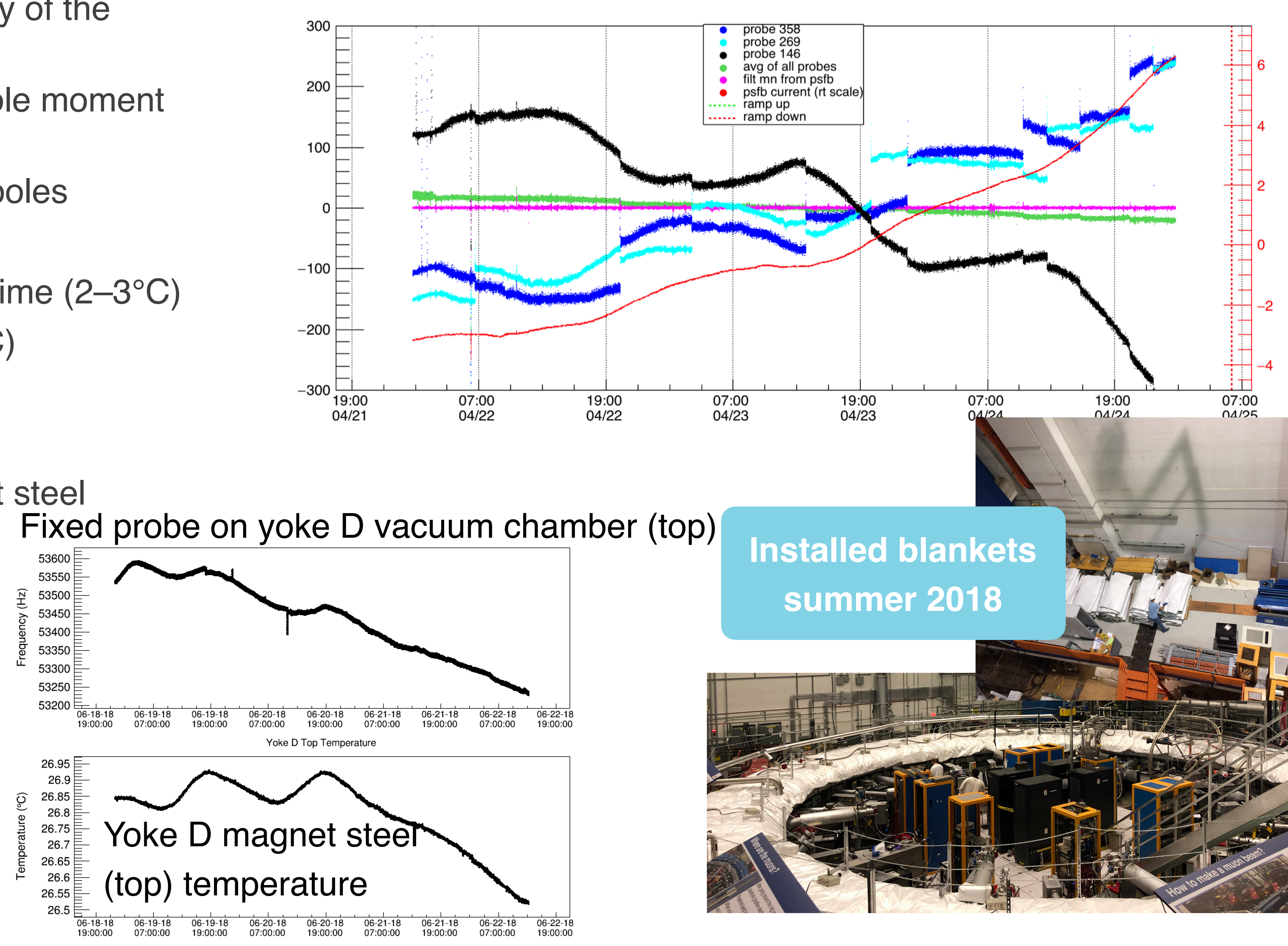

88 David Flay I Measuring the Muon Anomalous Magnetic Moment to High Precision

UMassAmherst 


\section{Plunging Probe Design}

- Used to calibrate the trolley NMR probes

- Symmetry is very important $=>$ minimizes field perturbations $=>$ reduced systematic uncertainties

- RF coil support: 15-mm OD high-precision glass cylinder

- Macor supports ensure alignment of RF coil (zero-X 0.97-mm OD wire)

- Ground shield: 1" OD, 1-mm wall 2024-T3 Al

- Stabilizes probe tune, reduces noise pickup
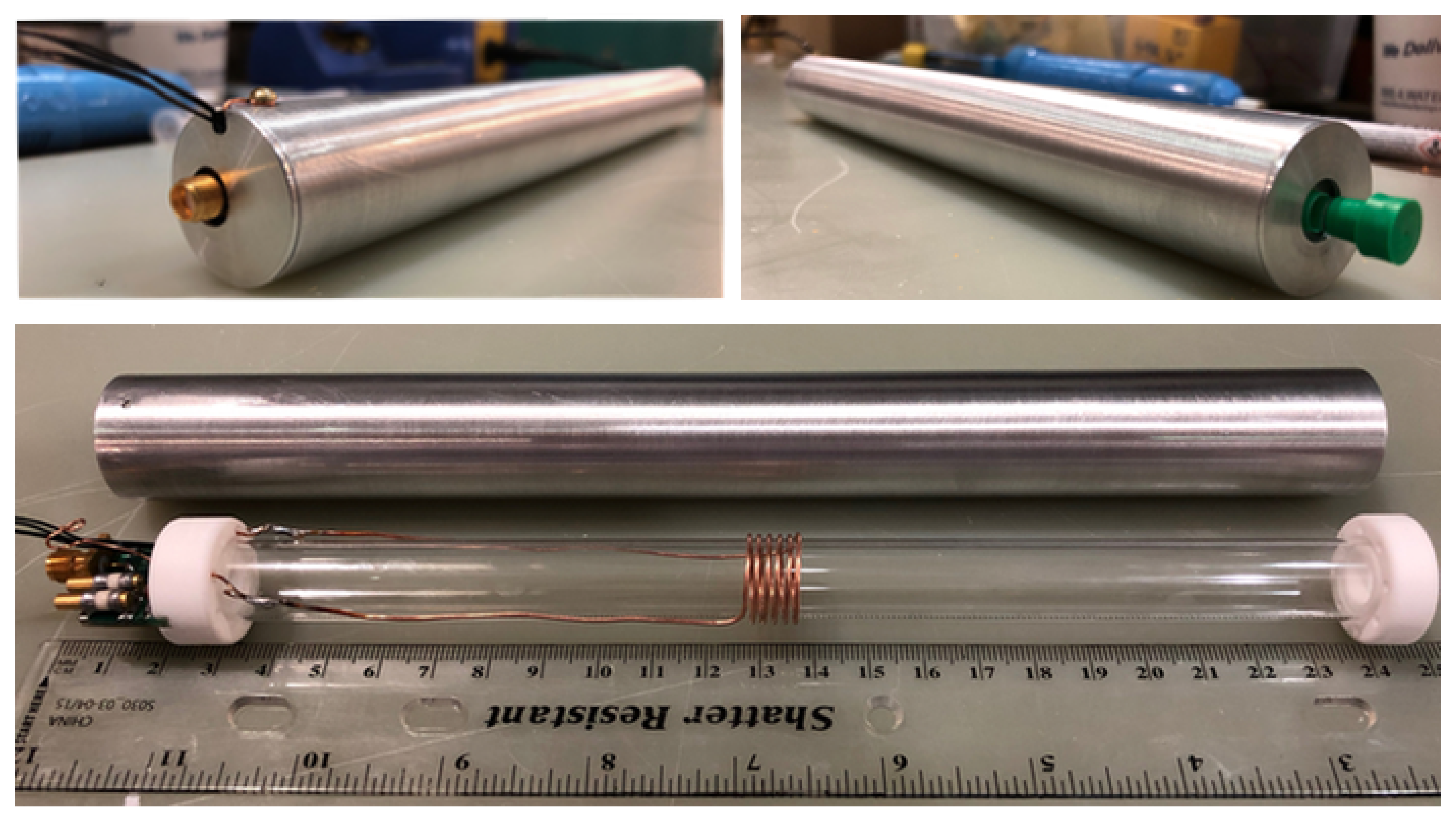

- Vacuum compatible

89 David Flay I Measuring the Muon Anomalous Magnetic Moment to High Precision 


\section{Plunging Probe: Measuring Perturbations}

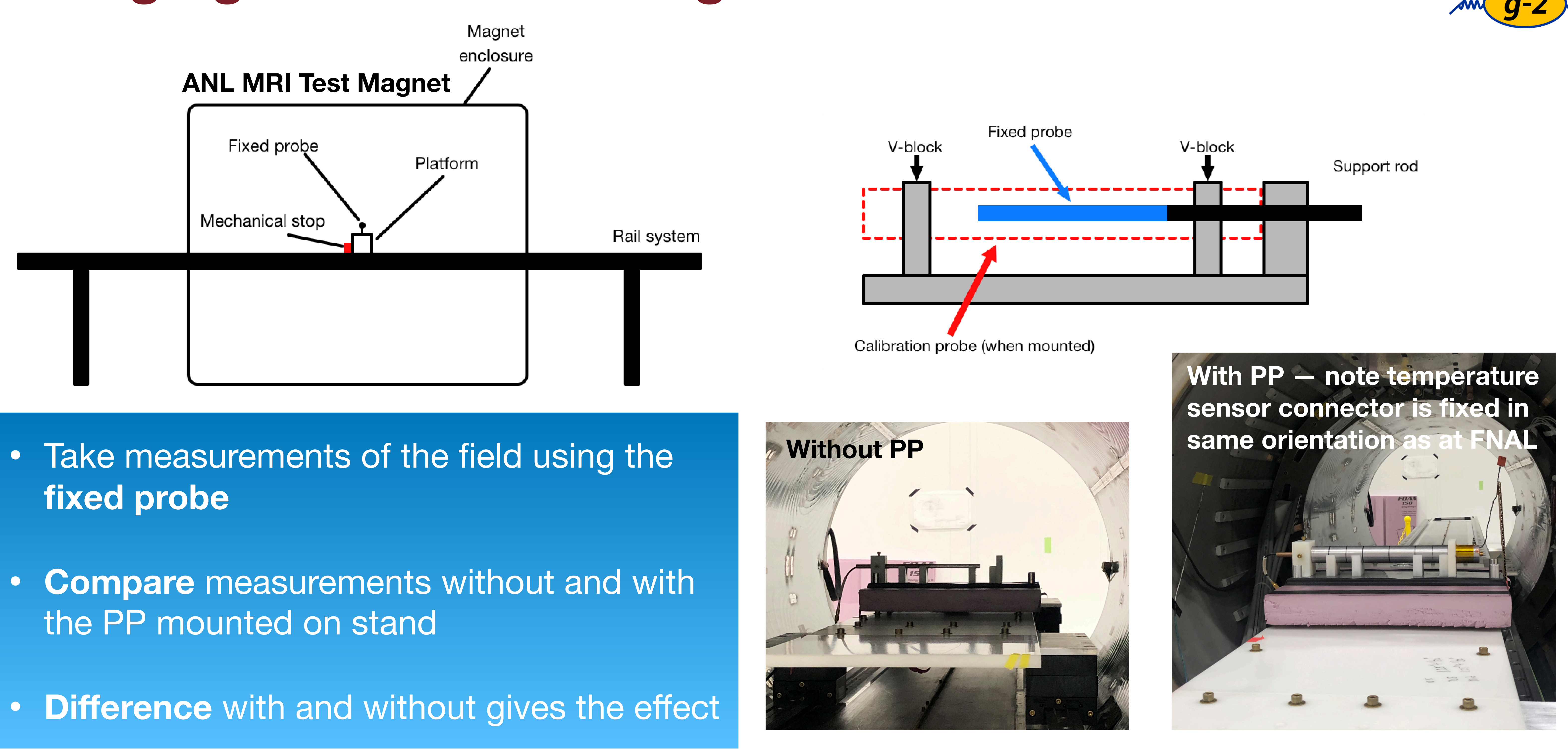

- Take measurements of the field using the fixed probe

- Compare measurements without and with the PP mounted on stand

- Difference with and without gives the effect

UMassAmherst

90 David Flay I Measuring the Muon Anomalous Magnetic Moment to High Precision 


\section{Plunging Probe: Magnetic Images}

- Need to account for the effect due to magnetic images of the PP in the pole pieces

- For an infinite plane with magnetic permeability $\mu_{r}$, the field due to the image of a material with perturbation $\Delta \mathrm{B}$ is:

$$
\Delta B^{\prime} \approx\left(\frac{\mu_{r}-1}{\mu_{r}+1}\right) \Delta B\left(x, y, z^{\prime}\right) \approx \Delta B\left(x, y, z^{\prime}\right)
$$

For $\mu_{\mathrm{r}}>>1\left(\sim 1450\right.$ for the magnet $\left.{ }^{\star}\right)$. Evaluate the perturbation at image distance $z$ ' in the pole piece (upper and lower)

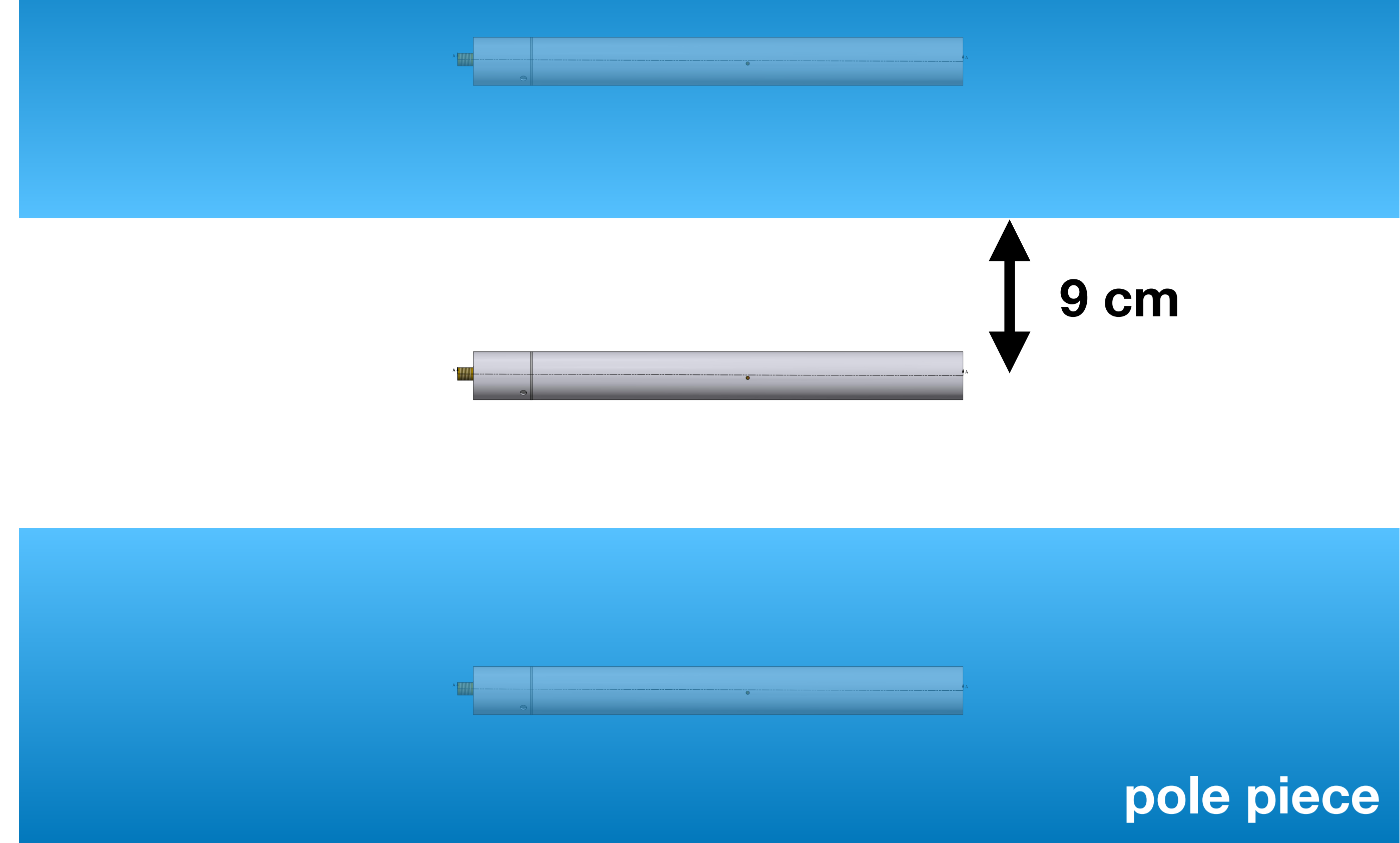




\section{Plunging Probe: Measuring the Images at FNAL}

- Use a stage to mount a fixed probe along the axis of the PP, which can slide over fixed probe

- Compare field measurements with and without PP installed on the stage

- Repeat measurements at height of center trolley probe, and highest trolley probe location

- Also conduct measurements with PP mounting rod attached/detached

Rod composition may not be pure aluminum (typically up to $\sim 20 \%$ variation in $x=>$ imperfect predictions)

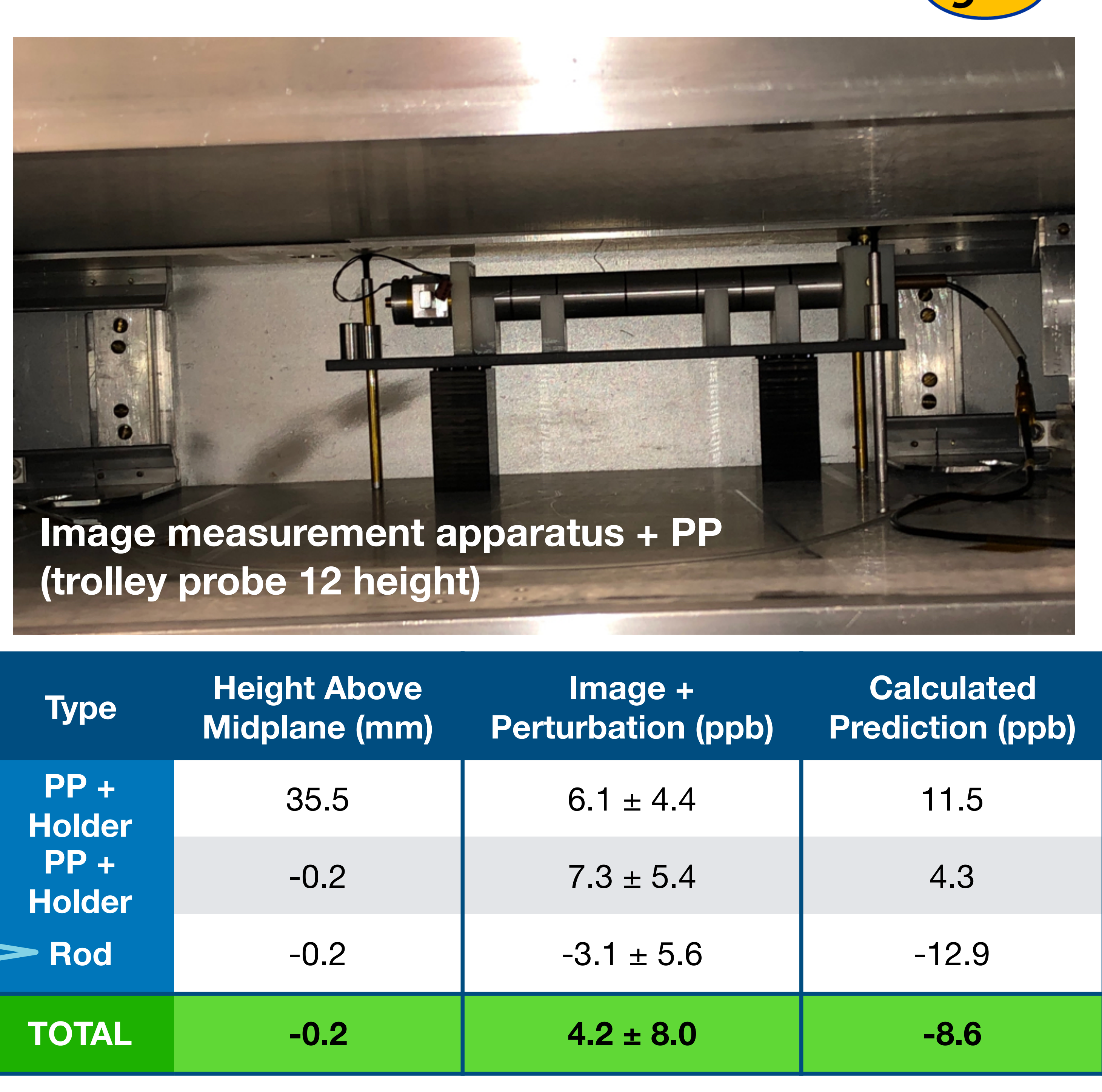




\section{Radiation Damping}

\section{What is it?}

- Precessing spins induce emf in pickup coil; this in turn generates an alternating magnetic field that acts to rotate spins back towards the main field

- Size of effect: $\delta_{R D} \sim\left[\left(f_{0}-f_{L}\right) / f_{0}\right] n Q M_{z}(t) / \tau_{R D}$

- $f_{0}=$ resonant frequency of circuit; $f_{L}=$ Larmor frequency

- $\eta$ = filling factor; $Q$ = quality factor of circuit

- $\mathrm{M}_{\mathrm{z}}(\mathrm{t})=$ magnetization of sample, $\mathrm{T}_{\mathrm{RD}}=$ time scale of effect

\section{How to quantify?}

- Use coils to produce a longitudinal field

- Precise control over main field to mimic damping effect

- Vary $\pi / 2$ pulse => vary $M_{z}(t)=>$ changes $\delta_{R D}$

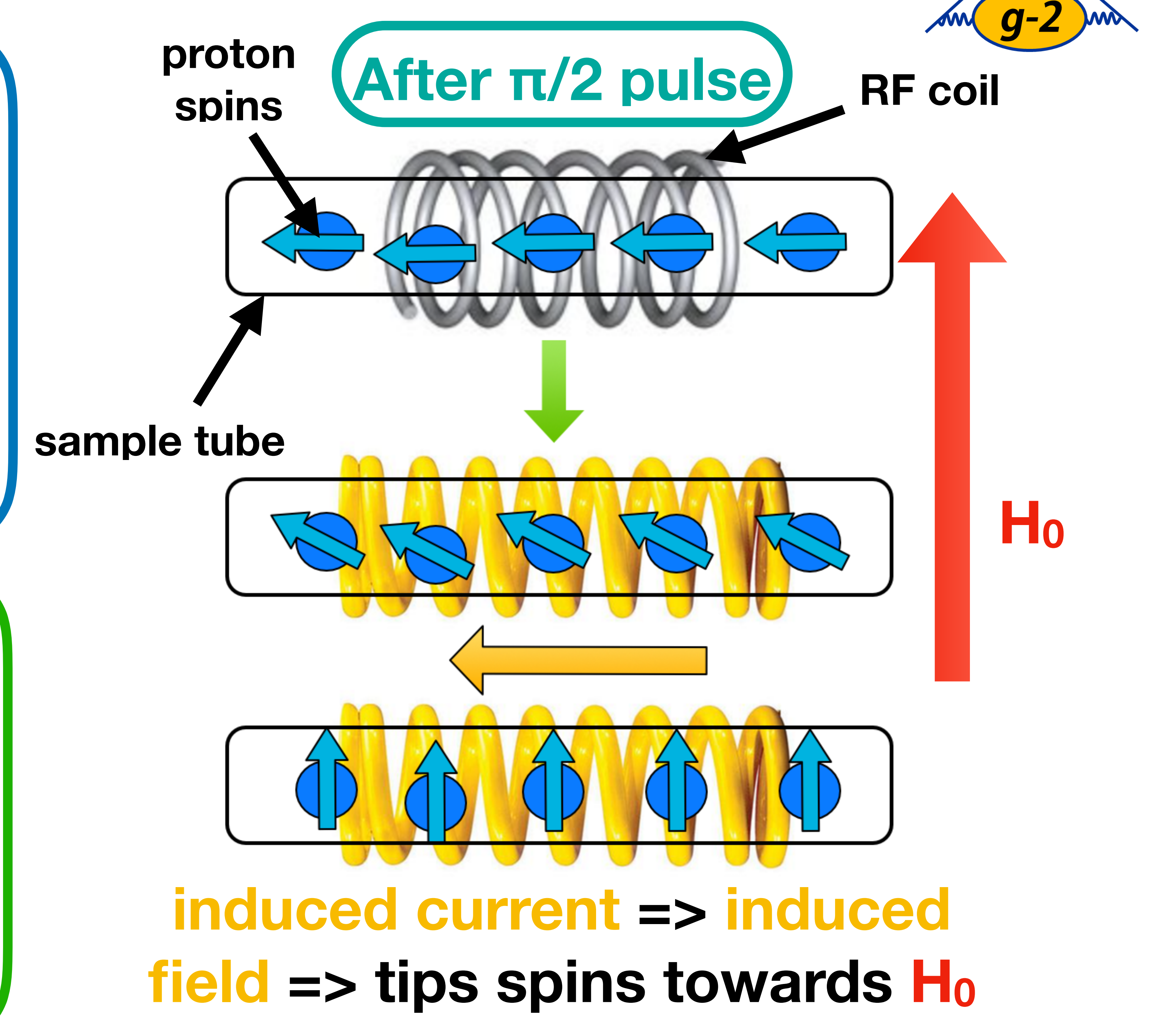

UMassAmherst 


\section{Calibrating the Trolley}

\section{Procedure}

- Select trolley probe to calibrate

- Impose a known gradient across the trolley; compare to bare field $B_{0}$. Define $\Delta B=B(I \neq 0)$ - $B(I=0)$

- Unique $\Delta \mathrm{B}$ for each trolley probe gives position

- Move plunging probe into volume; measure $\Delta \mathrm{B}$ and determine distance to move plunging probe

- Iterate until plunging probe $\Delta \mathrm{B}$ matches trolley probe $\Delta \mathrm{B}$

- Perform for radial, vertical, azimuthal coordinates
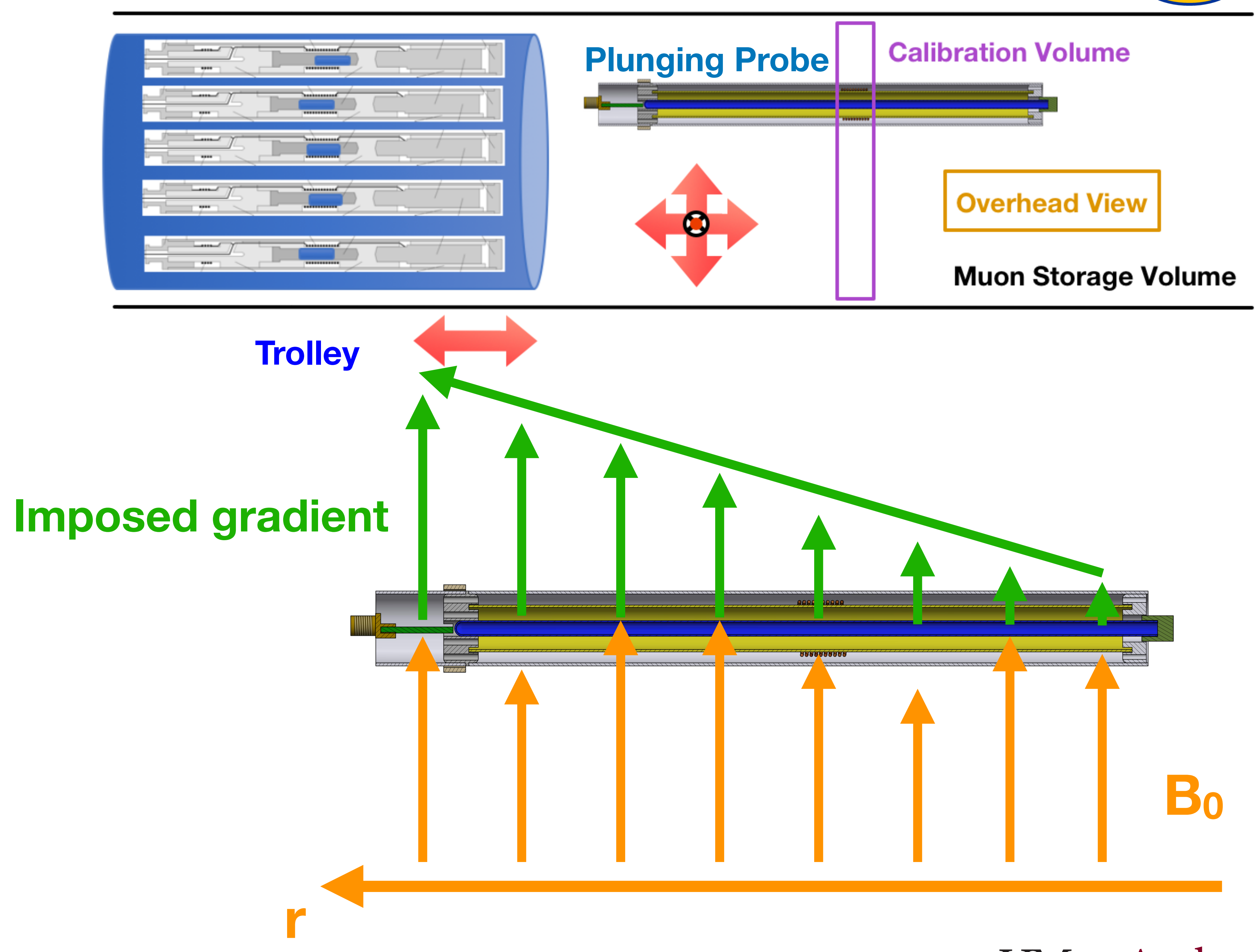

UMassAmherst

98 David Flay I Measuring the Muon Anomalous Magnetic Moment to High Precision 


\section{Calibrating the Trolley}

\section{Procedure}

- Select trolley probe to calibrate

- Impose a known gradient across the trolley; compare to bare field $B_{0}$. Define $\Delta B=B(l \neq 0)$ - $B(I=0)$

- Unique $\Delta \mathrm{B}$ for each trolley probe gives position

- Move plunging probe into volume; measure $\Delta \mathrm{B}$ and determine distance to move plunging probe

- Iterate until plunging probe $\Delta \mathrm{B}$ matches trolley probe $\Delta \mathrm{B}$

- Perform for radial, vertical, azimuthal coordinates

- Shim the field to be highly uniform, and measure using the PP and the trolley (rapid swapping)
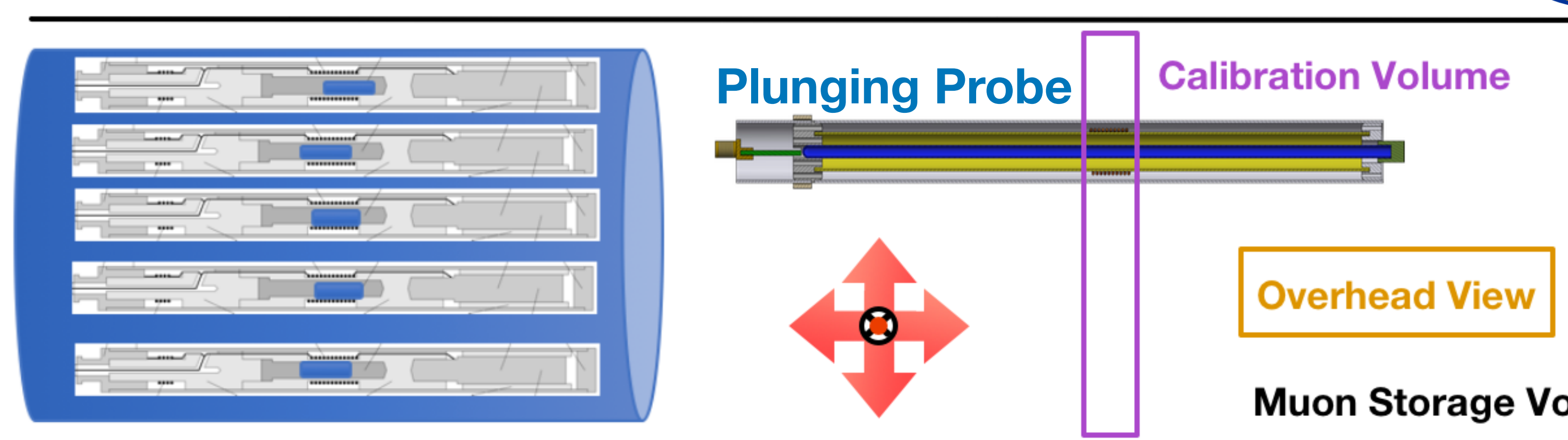

Trolley

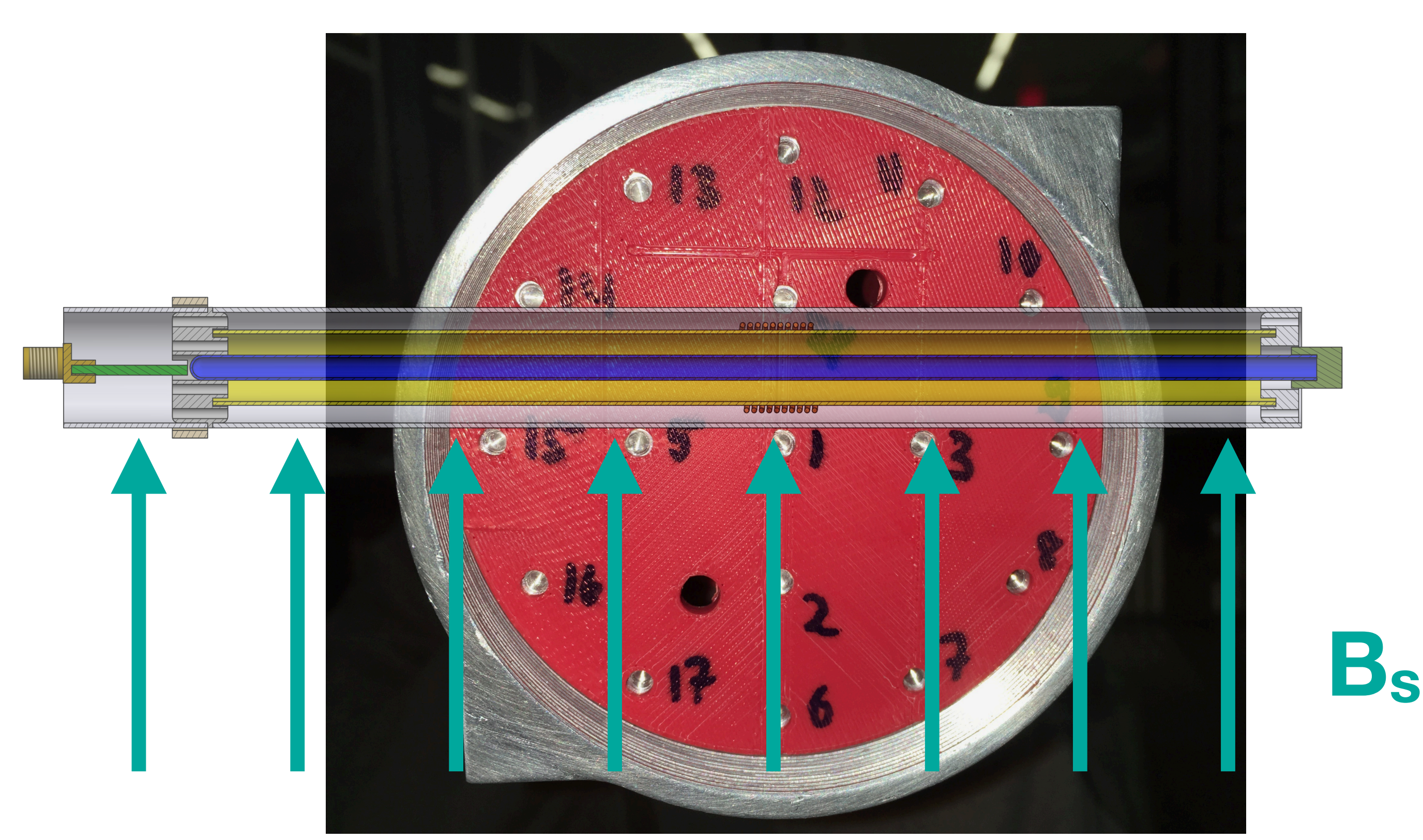

